\title{
Projection-operator methods for classical transport in magnetized plasmas. Part 1. Linear response, the Braginskii equations and fluctuating hydrodynamics
}

\author{
John A. Krommes $\dagger$ \\ Princeton Plasma Physics Laboratory, P. O. Box 451, MS 28, Princeton, New Jersey 08543-0451, USA
}

(Received 18 January 2018; revised 22 May 2018; accepted 23 May 2018)

\begin{abstract}
An introduction to the use of projection-operator methods for the derivation of classical fluid transport equations for weakly coupled, magnetised, multispecies plasmas is given. In the present work, linear response (small perturbations from an absolute Maxwellian) is addressed. In the Schrödinger representation, projection onto the hydrodynamic subspace leads to the conventional linearized Braginskii fluid equations when one restricts attention to fluxes of first order in the gradients, while the orthogonal projection leads to an alternative derivation of the Braginskii correction equations for the non-hydrodynamic part of the one-particle distribution function. The projection-operator approach provides an appealingly intuitive way of discussing the derivation of transport equations and interpreting the significance of the various parts of the perturbed distribution function; it is also technically more concise. A special case of the Weinhold metric is used to provide a covariant representation of the formalism; this allows a succinct demonstration of the Onsager symmetries for classical transport. The Heisenberg representation is used to derive a generalized Langevin system whose mean recovers the linearized Braginskii equations but that also includes fluctuating forces. Transport coefficients are simply related to the two-time correlation functions of those forces, and physical pictures of the various transport processes are naturally couched in terms of them. A number of appendices review the traditional Chapman-Enskog procedure; record some properties of the linearized Landau collision operator; discuss the covariant representation of the hydrodynamic projection; provide an example of the calculation of some transport effects; describe the decomposition of the stress tensor for magnetised plasma; introduce the linear eigenmodes of the Braginskii equations; and, with the aid of several examples, mention some caveats for the use of projection operators.
\end{abstract}

Key words: plasma classical transport

$†$ Email address for correspondence: krommes@princeton.edu 
Contents

$\begin{array}{lll} & \text { Introduction } & 3\end{array}$

2 Linearized hydrodynamics for the one-component plasma $\quad 7$

2.1 Basic idea of the hydrodynamic projection . . . . . . . . . . . . 7

2.2 Transport equations for the unmagnetised, one-component, weakly

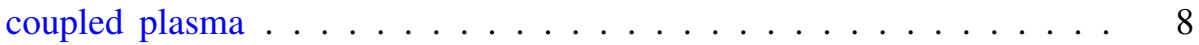

2.2.1 Definition of the projection operator . . . . . . . . . 11

2.2.2 Projecting the kinetic equation; the statistical closure problem . 14

3 The Braginskii equations: Multispecies, magnetised, classical, collisional fluid equations

3.1 Exact form of the moment equations, and summary of the results for a two-species plasma . . . . . . . . . . . . . 20

3.2 The hydrodynamic projection for multispecies and magnetised plasma 22

3.3 Hydrodynamic closure . . . . . . . . . . . . . . . . . 25

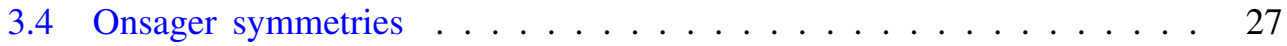

4 Generalized Langevin equation for the hydrodynamics of magnetised plasmas

4.1 Heisenberg versus Schrödinger representations . . . . . . . . . . . . 30

4.2 Derivation of the generalized Langevin equation . . . . . . . . . . . . . . 31

4.3 Fluctuating hydrodynamics and transport coefficients . . . . . . . . 33

4.3.1 Transport coefficients as current-current correlations . . . . . . 34

4.3.2 Fluctuating forces and collision-driven fluxes . . . . . . . 34

5 Discussion $\quad 36$

Appendix A. The traditional Chapman-Enskog calculation for the onecomponent plasma $\quad 38$

A.1 The kinetic time scale . . . . . . . . . . . . . . . . . 39

A.2 The Transit time scale .................. . . . . . . . . . . . 40

A.3 The hydrodynamic regime . . . . . . . . . . . . . 42

Appendix B. The linearized Landau operator 44

B.1 Properties of the linearized Landau operator . . . . . . . . . . . . . . . 47

B.2 Calculation of $\widehat{\mathrm{PCP}}|\Delta \chi\rangle \ldots \ldots \ldots \ldots$

B.3 Calculation of $\widehat{\mathrm{CP}}|\Delta \chi\rangle \ldots \ldots \ldots$. . . . . . . . . . 49

B.4 The spectrum of the linearized Landau operator and its relation to the Markovian approximation ............... 49

Appendix C. Covariant representation of the hydrodynamic projection 52

Appendix D. An example of the calculation of some transport coefficients: classical electron heat flow

Appendix E. Decomposition of the stress tensor 
Appendix F. Linear eigenmodes of the Braginskii equations

F.1 Eigenmodes of the unmagnetised one-component plasma . . . . . . 58

F.2 Eigenmodes of a two-species magnetised plasma for $\boldsymbol{B}=\mathbf{0} \ldots \ldots 1$

F.3 Eigenmodes of a two-species magnetised plasma for $v /\left|\omega_{c}\right| \ll 1 \ldots 62$

F.3.1 Magnetized eigenvalues in the limit of zero dissipation . . . 63

F.3.2 Magnetized eigenvalues for purely perpendicular propagation with no dissipation . . . . . . . . . . . . . 63

F.3.3 Magnetized eigenvalues for purely perpendicular propagation with dissipation . . . . . . . . . . . . . . 64

Appendix G. Projection operators: caveats and further examples $\quad \mathbf{6 6}$

G.1 Projection-operator methods and non-locality . . . . . . . . . . 66

G.2 Projection operators and the dispersion relation of Langmuir oscillations 68

G.3 The plateau phenomenon; unmodified vs modified propagators . . . . 71

G.4 Projection-operator analysis of the Brownian test particle . . . . . . 75

G.4.1 Projection into the density subspace . . . . . . . . 76

G.4.2 Projection into a multidimensional subspace . . . . . . 76

\section{Introduction}

The review article by Braginskii (published in Russian in 1963 and in English translation in 1965) on classical transport in weakly coupled, magnetised, multispecies plasmas has served as an invaluable reference for multiple generations of plasma physicists. For a two-component plasma with small electron-to-ion mass ratio, $\mu \doteq m_{e} / m_{i} \ll 1$ ( $\doteq$ denotes a definition), Braginskii described a path to an approximate derivation of the so-called correction equations for the non-hydrodynamic parts of the distribution function, from which the classical transport coefficients are ultimately derived. The methodology, first published by Braginskii (1957), ${ }^{1}$ can be traced back to the pioneering work of Chapman (1916) and Enskog (1917) on the kinetic theory of rarefied gases; for many details, see Chapman \& Cowling (1952). The traditional Chapman-Enskog procedure is reviewed for the simple case of a one-component plasma (OCP) in appendix A.

Although the relevant mathematics was described clearly by Braginskii (1957), experience shows that many students do not take the time to work through those calculations and consequently do not always grasp the beautiful underlying structure of the transport problem. The techniques described in this article provide an alternative, heuristically appealing, and technically efficient approach that for neutral fluids is known to unify a number of threads of non-equilibrium statistical mechanics. That unification carries over to the more complicated plasma. Appreciation of the methods enables one to avoid reinventing the wheel and provides one with a concise yet workable formalism on which non-trivial generalizations can be built. An example of such a generalization is the calculation of second-order (Burnett) transport coefficients, addressed in Part 2 of this series of articles (Krommes 2018b).

In the same year that the English translation of Braginskii's review appeared, Mori (1965) published a seminal paper in which the transport problem was reformulated with the aid of projection-operator methods. The general approach had been anticipated by Zwanzig (1961a,b), and the methodology is now known as the Mori-Zwanzig formalism. The purpose of the present article is to describe the application of the Mori-Zwanzig formalism to the problem of classical transport in

\footnotetext{
${ }^{1}$ Braginskii's original 1957 paper contains a footnote indicating that the work was performed in 1952.
} 
weakly coupled, magnetised plasmas. This leads one to an alternative derivation of the Braginskii correction equations.

A great strength of Braginskii's review article is its focus on the physical interpretation of the transport coefficients, knowledge of which is essential for researchers on magnetised plasmas. But although Braginskii's interpretations of the mathematics are entirely correct, here too the present projection methods are helpful in providing additional intuition. They enable an extension of Braginskii's equations for the macroscopic, mean hydrodynamic variables (density, flow, and temperature) to generalized Langevin equations that include fluctuating forces. Such equations have previously been derived from the Bogoliubov-Born-Green-Kirkwood-Yvon (BBGKY) hierarchy (Bixon \& Zwanzig 1969; Hinton 1970), but the projection-operator methods are arguably more transparent and efficient. Fluctuating forces appear implicitly in Braginskii's heuristic explanations of the various transport processes, and their effects are contained in his systematic mathematics. However, explicit definition of those forces brings additional clarity to the transport calculations.

A projection operator $\mathrm{P}$ is linear and idempotent (i.e. $\mathrm{P}^{2}=\mathrm{P}$ ). It extends to linear algebra and functional analysis the notion of graphical projection onto an axis. An example of a projection operator is the ensemble average ${ }^{2}\langle\ldots\rangle$. Thus, all of statistical closure theory (Krommes 2002, 2015) can be said to involve projection operators. However, this is stretching the point. The specifically Mori-Zwanzig-style projection-operator techniques have a particular flavour and do not naturally generalize to all possible methods of statistical closure. They have been little used in plasma physics. Krommes (1975) and Krommes \& Oberman (1976) employed them to discuss the phenomenon of long-time tails in magnetised, thermal-equilibrium plasmas, earlier identified in the molecular-dynamics neutral-fluid computer experiments of Alder \& Wainwright (1970). ${ }^{3}$ They are mentioned briefly by Diamond, Itoh \& Itoh (2010, $\S 6.2$ ). However, they have not been used systematically in the context of plasma transport theory. In particular, there is no published account of their application to the derivation of the Braginskii transport equations. ${ }^{4}$

One possible reason that the Mori-Zwanzig formalism has not seen much use in plasma physics is that the most general non-equilibrium statistical-mechanical treatments of the transport problem produce formulas for the transport coefficients in terms of averages over the $N$-particle equilibrium ensemble and dynamics evolved with a modified $N$-particle Liouville propagator, where $N$ is the total number of particles in the system. Such formulas go back to Green (1954) and Kubo (1957). (Subtleties with the interpretation of the Green-Kubo formulas are discussed in $\S$ G.3.) While elegant, they are difficult to work out in the general case. However, in the special but very important regime of weak coupling, those formulas simplify dramatically. Moreover, with the further approximation of linear response an alternative approach becomes possible wherein a kinetic equation for the one-particle distribution function is first derived, then processed to produce formulas for the transport coefficients. It is

\footnotetext{
${ }^{2}$ Indeed, any linear, properly normalized averaging operation $\mathrm{A}$ possesses the property $\mathrm{A}^{2}=\mathrm{A}$ and is thus a projection operator. Weinstock $(1969,1970)$ employed such operators in his approach to Vlasov turbulence; see some discussion by Krommes (2002) and Krommes (2015). Weinstock's work was pursued, for example, by Misguich \& Balescu (1975) and Misguich (1975).

${ }^{3}$ Some discussions of long-time tails are given by Balescu (1975, §21.5), Reichl (1998, § 11.A) and Zwanzig (2001, chap. 9).

${ }^{4}$ Some of the material discussed here was taught by the author for many years in the course Irreversible Processes in Plasma offered at the second-year graduate level in Princeton University's Department of Astrophysical Sciences. One purpose of this article is to make this material more accessible and to suggest that it is worthy of a core topic in a plasma-physics educational curriculum.
} 
that processing that is clarified by the projection-operator techniques described in the present paper. I shall show that a suitably modified Mori-Zwanzig formalism can be easily applied to the linearized plasma kinetic equation, leads efficiently to standard results, clarifies the structure of the hydrodynamic system and fosters heuristic understanding of the first-order transport processes.

By $n$ th-order processes, I mean contributions to dissipative fluxes of $n$th order in the gradients. Thus, 'first order' is to be distinguished from 'linear response'. The latter describes a formally infinitesimal perturbation of thermal equilibrium. In either linear response theory or a more general nonlinear theory, dissipative fluxes exist that are driven by arbitrary powers of the gradients. The proportionality factors are the ' $n$ th-order transport coefficients' (more precisely, the order-unity transport coefficients that enter in fluxes involving $n$ th-order gradients). The first-order coefficients are called the Braginskii transport coefficients by plasma physicists; they are known in non-equilibrium statistical mechanics as the Navier-Stokes coefficients. For example, if small perturbations are denoted by $\Delta$, the first-order heat flux in an unmagnetised plasma has the form $\Delta \boldsymbol{q}=-n \kappa \nabla \Delta T$ ( $n$ is the density and $T$ is the temperature); $\kappa$ is the first-order thermal conductivity. This relation is both linear $(\Delta \boldsymbol{q} \propto \Delta T)$ and first order $(\Delta \boldsymbol{q} \propto \nabla)$. The second-order coefficients are called the Burnett transport coefficients. Some Burnett coefficients exist already in the linear approximation. For example, one contribution to the linear Burnett heat flux has the form $\Delta \boldsymbol{q}=n \kappa^{\prime} \nabla^{2} \Delta \boldsymbol{u}$, where $\boldsymbol{u}$ is the fluid velocity. For nonlinear response, additional Burnett terms arise. For example, a contribution to the nonlinear Burnett heat flux has the form $\Delta \boldsymbol{q}=n \kappa^{\prime \prime}(\nabla \cdot \Delta \boldsymbol{u}) \nabla \Delta T$.

This paper is Part 1 of a two-part series of articles whose goal is to show how to formulate classical, weakly coupled plasma transport theory by using projection-operator methods. Part 1 describes a self-contained rederivation of the Braginskii correction equations for the special case of linear perturbations of an absolute Maxwellian equilibrium. This is less than what Braginskii accomplished (his equations are nonlinear), but it serves to familiarize one with the basic methodology in the simplest possible context. (Following Braginskii, I restrict my attention to effects of first order in the gradients. Although the present methodology could also be used to study linear Burnett effects, I shall defer such calculation to the more general discussion given in Part 2, where nonlinear Burnett effects are also considered.) I shall also derive a set of generalized Langevin equations that extend Braginskii's equations to include fluctuating forces. That is a core topic that provides important insight into the structure of the transport theory.

In Part 2, the problem is reformulated in order to embrace nonlinearity and arbitrarily strong coupling. The methodology used there is a generalization of the formulation of Brey, Zwanzig \& Dorfman (1981) to include a background magnetic field and multiple species. The nonlinear Braginskii equations are recovered with ease. The techniques also allow one to obtain the next-higher-order Burnett equations, including both of the linear and nonlinear effects as described above. Those were originally obtained for plasmas described by the Landau collision operator by Mikhǎ̌lovskiǐ (1967), Mikhǎlovskiı̌ \& Tsypin (1971, 1984) and Catto \& Simakov (2004), none of whom remarked on connections to previous calculations of Burnett coefficients for neutral gases. In Part 2 it is shown that the Navier-Stokes and Burnett transport coefficients calculated by Catto and Simakov are special cases of the complete set of coefficients for which Brey et al. (1981) gave general expressions in terms of integrals of two-time correlation functions. ${ }^{5}$ Thus, the present series of articles serves to unify a variety of previous research threads.

${ }^{5}$ A proviso is that Brey et al. (1981) did not include a magnetic field, but that is easy to add. 
I now return to an overview of the present Part 1. I illustrate the basic approach in $\S 2$ by deriving linearized transport equations for the unmagnetised one-component plasma, restricting attention to dissipative fluxes that are of first order in the gradients. Then in § 3 I extend the calculations to embrace multispecies and magnetised plasmas, and I derive the linearized version of Braginskii's correction equations.

The formalism employed in $\S \S 2$ and 3 uses what is known as the Schrödinger representation, which means that one takes time-independent velocity moments of the time-dependent distribution function $f$ (which is taken to evolve according to the Landau kinetic equation). Alternatively, one can use the Heisenberg representation, in which equations are written for time-dependent, random hydrodynamic observables whose statistical behaviour evolves from statistics (velocity dependence) that reside in the distribution function at some initial time. The averaged equations are the same as before. However, the raw equations for the random variables can be cast into the form of generalized Langevin equations, which include fluctuating forces and imply a theory of hydrodynamic correlation functions. This is done in $\S 4$. The body of the paper concludes with a brief discussion in $\S 5$.

Several appendices are included. In appendix A I review the traditional ChapmanEnskog calculation for the one-component plasma, in appendix B I record various properties of the linearized Landau collision operator, in appendix C I describe a technically efficient covariant representation of the transport equations, in appendix D I provide an example of the evaluation of the general formulas by considering the electron heat flow in the limit of small collisionality, in appendix E I review Braginskii's tensorial decomposition of the stress tensor, in appendix F I consider some important special cases of the linear eigenmodes of the Braginskii equations and in appendix G I focus on some important caveats regarding the use of projection operators. Key notation is summarized in appendix 2:I, ${ }^{6}$ which merges the definitions from both Part 1 and Part 2.

This paper is intended to be useful to both graduate students just beginning their study of classical plasma transport as well as seasoned researchers interested in advanced techniques. Although it rederives some of Braginskii's principal results and quotes others of them for completeness, it is neither a complete replacement for Braginskii's article nor a comprehensive review of classical plasma transport theory. For initial study of classical transport, a reasonable plan of attack would be to begin by skimming the first half of Braginskii (1965) (through the end of $\$ 5$, p. 262). Additional perspective should then be provided from the present paper by $\S \S 1-3$ and 5 as well as appendices A, B, D, E and F, with $\S 4$ and appendices C and G containing more advanced material. To fully appreciate the material in appendix G, the reader may find it useful to first study the concise modern introduction to non-equilibrium statistical mechanics given by Zwanzig (2001), particularly chap. 8 on projection operators.

The material in Part 1 is intended to be introductory, and the restrictions to linear response and first-order transport coefficients are greatly simplifying. In fact, some deep conceptual issues lurk in what may appear at first glance to be straightforward analysis. If concerns arise, consult $\S 5$ for brief discussion and see Part 2 for a more thorough treatment.

\footnotetext{
${ }^{6}$ Sections relating to Part 2 are prefaced by '2:' (e.g. appendix 2:H).
} 


\section{Linearized hydrodynamics for the one-component plasma}

In a one-component, weakly coupled ${ }^{7}$ plasma (for example, a discrete ion plasma with smooth electron neutralizing background) in which the collision frequency $v$ is ordered large, it is well known that Chapman-Enskog theory singles out the number density, momentum density, and kinetic-energy density (or temperature, or entropy density) as preferred hydrodynamic variables. That is, those quantities are conserved by the Landau collision operator. Mathematically, with $\bar{n}$ being the spatially uniform mean density, the velocity-dependent functions $\bar{n}, \bar{n} m \boldsymbol{v}$, and $(1 / 2) \bar{n} m v^{2}$ are the left null eigenfunctions of the collision operator (in terms of a natural scalar product to be defined shortly). No dissipative transport is associated with the null eigenfunctions. Rather, transport is carried by the non-hydrodynamic corrections to the local Maxwellian distribution function. The traditional Chapman-Enskog approach is reviewed in appendix A.

\subsection{Basic idea of the hydrodynamic projection}

These ideas can be codified by dividing a complete set of functions on the single-particle velocity space into two orthogonal subspaces: the hydrodynamic subspace $\mathcal{H}$, spanned by the null eigenfunctions of the collision operator, and the non-hydrodynamic, orthogonal, or vertical subspace $O .{ }^{8}$

The fluid evolution equations 'live in the hydrodynamic subspace'. The nonhydrodynamic part of the distribution function determines the values of the transport coefficients. Transport coefficients enter the fluid equations because the dynamics of the hydrodynamic and non-hydrodynamic subspaces are coupled by the evolution equation for $f$. That coupling is a special case of the statistical closure problem (Krommes 2015) for passive equations with random coefficients. (Such equations are said to possess a stochastic nonlinearity.) Here the random variable is velocity and the stochastic nonlinearity is the $\boldsymbol{v} \cdot \nabla f$ term in the kinetic equation.

The decomposition into hydrodynamic and non-hydrodynamic parts can be expressed by the introduction of appropriate projection operators. If $\mathrm{P}$ projects into $\mathcal{H}$ and $\mathrm{Q} \doteq 1-\mathrm{P}$ (where 1 is the identity operator) therefore projects into $O$, and if the perturbed (denoted by $\Delta$ ) distribution function is written as $\Delta f=\Delta f_{\mathrm{h}}+\Delta f_{\perp}$, then $\Delta f_{\mathrm{h}}=\mathrm{P} \Delta f$ and $\Delta f_{\perp}=\mathrm{Q} \Delta f$. The basic idea is illustrated in figure 1; the precise realization of $\mathrm{P}$ is given in the next section.

The formulation described in the present paper focuses on the decomposition of the one-particle distribution function $f_{s}(\boldsymbol{x}, \boldsymbol{v}, t)$, which lives in the so-called $\mu$ space (the six-dimensional (6-D) phase space for one generic particle of species $s$ ). In contrast, the instantaneous state of the entire collection of $N$ particles can be described by a single point in the so-called $\Gamma$ space (of $6 N$ dimensions). A well-known consequence of the reduction from $\Gamma$ space to $\mu$ space is that the kinetic evolution equation for $f$ is nonlinear (it involves the product $\boldsymbol{E}[f] f$, where the electric field $\boldsymbol{E}$ is a linear functional of $f$, as well as a nonlinear collision operator), which leads to various complications. $\Gamma$-space dynamics, on the other hand, is formally linear, being described by the Liouville equation. That linearity combined with the linearity of projection operators was usefully exploited by Mori, who was able to write exact equations for two-time correlation functions with apparent ease; ultimately, the

\footnotetext{
${ }^{7}$ Weakly coupled means that the plasma is almost an ideal gas (i.e. that the plasma discreteness parameter $\epsilon_{\mathrm{p}} \doteq 1 /\left(n \lambda_{\mathrm{D}}^{3}\right)$ is very small). Here $\lambda_{\mathrm{D}}$ is the Debye length.

${ }^{8}$ The notion of a vertical projection is common in the modern theory of differential geometry; see, for example, Fecko (2006).
} 


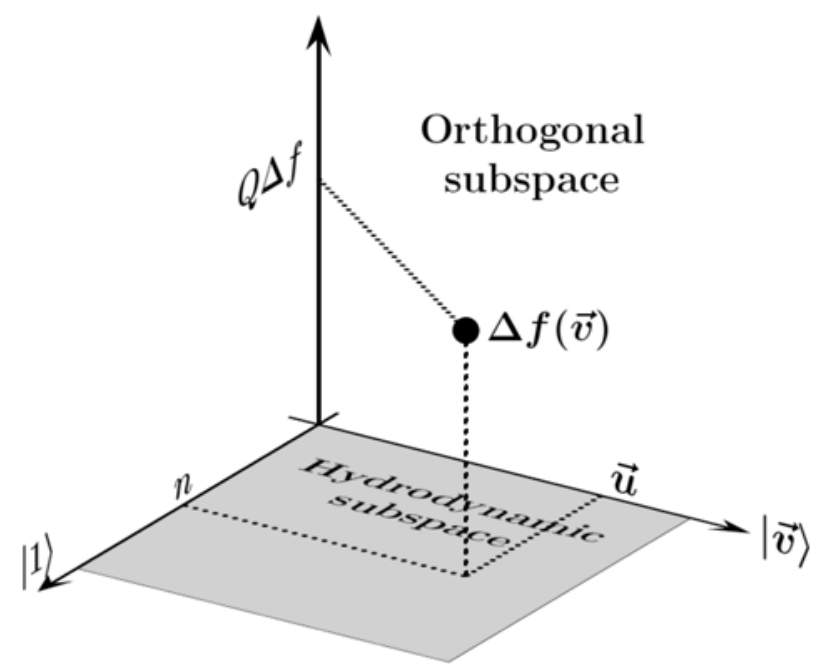

FIgURE 1. Illustration of the $\mathrm{P}$ and $\mathrm{Q}$ projections. The kinetic-energy axis of the hydrodynamic subspace is omitted for clarity.

transport coefficients are expressed in terms of various integrals over those correlation functions. However, the simplicity is only formal. Mori's projection operators involve the full $N$-particle dynamics, and his equations contain nonlinearities to all orders; in the general case, it is difficult to extract from them quantitative expressions for the transport coefficients. As I shall show in Part 2, the formulas simplify in the limit of weak coupling, which fortunately is appropriate for research on magnetic fusion and various other applications. However, that still leaves the dichotomy between the linearity of projection operators and the nonlinearity of $\mu$-space dynamics to be dealt with. It might seem that a projection-operator formalism is restricted to the derivation of linearized transport equations. That is not correct, although the generalization is non-trivial. I shall address nonlinear transport in Part 2. In the present paper, I restrict my attention to linear response, where the basic features of the projection-operator methodology can be explained in the simplest possible context.

\subsection{Transport equations for the unmagnetised, one-component, weakly coupled plasma}

In this section I shall introduce the formalism by using projection methods to derive the linearized fluid equations for the one-component plasma (OCP) in the limit of weak coupling and in the electrostatic approximation with background magnetic field $\boldsymbol{B}=\mathbf{0}$, restricting my attention to fluxes that are of first order in the gradients. (An even simpler example, the Brownian test particle, is discussed in §G.4.) It is well known and easy to show that the fully nonlinear moment equations for the density $n$, flow velocity $\boldsymbol{u}$, and temperature $T$ of the one-component, weakly coupled plasma are

$$
\begin{aligned}
& \partial_{t} n(\boldsymbol{x}, t)+\nabla \cdot(n \boldsymbol{u})=0, \\
& n m \frac{\mathrm{d} \boldsymbol{u}(\boldsymbol{x}, t)}{\mathrm{d} t}=n q \boldsymbol{E}-\nabla p-\nabla \cdot \boldsymbol{\pi}, \\
& \frac{3}{2} n \frac{\mathrm{d} T(\boldsymbol{x}, t)}{\mathrm{d} t}=-p \nabla \cdot \boldsymbol{u}-\nabla \cdot \boldsymbol{q}-(\boldsymbol{\nabla} \boldsymbol{u}): \boldsymbol{\pi} .
\end{aligned}
$$


Here $^{9}$

$$
\begin{aligned}
& n(\boldsymbol{x}, t) \doteq \int \mathrm{d} \boldsymbol{v} \bar{n} f(\boldsymbol{x}, \boldsymbol{v}, t), \\
& n \boldsymbol{u}(\boldsymbol{x}, t) \doteq \int \mathrm{d} \boldsymbol{v} \bar{n} \boldsymbol{v} f(\boldsymbol{x}, \boldsymbol{v}, t), \\
& \frac{3}{2} n T(\boldsymbol{x}, t) \doteq \int \mathrm{d} \boldsymbol{v} \frac{1}{2} \bar{n} m w^{2} f(\boldsymbol{x}, \boldsymbol{v}, t),
\end{aligned}
$$

where $\boldsymbol{w} \doteq \boldsymbol{v}-\boldsymbol{u}$ is the so-called peculiar velocity and $p \doteq n T$ is the pressure of an ideal gas. The total advective time derivative is

$$
\frac{\mathrm{d}}{\mathrm{d} t} \doteq \frac{\partial}{\partial t}+\boldsymbol{u} \cdot \nabla,
$$

and the stress tensor $\pi$ and the heat-flow vector $\boldsymbol{q}$ are defined by

$$
\begin{aligned}
\boldsymbol{\pi} & \doteq \int \mathrm{d} \boldsymbol{v}(\bar{n} m \boldsymbol{w}) \boldsymbol{w} f-p \boldsymbol{I} \\
\boldsymbol{q} & \doteq \int \mathrm{d} \boldsymbol{v}\left(\frac{1}{2} \bar{n} m w^{2}\right) \boldsymbol{w} f
\end{aligned}
$$

These equations are closed in the collisional limit by the results

$$
\boldsymbol{\pi} \doteq-n m \mu\left((\nabla \boldsymbol{u})+(\nabla \boldsymbol{u})^{\mathrm{T}}-\frac{2}{3}(\nabla \cdot \boldsymbol{u}) \boldsymbol{l}\right),
$$

where $\mathrm{T}$ denotes transpose and $\mu$ is the kinematic viscosity ${ }^{10}$; and

$$
\boldsymbol{q} \doteq-n \kappa \nabla T
$$

where $\kappa$ is the first-order thermal conductivity. The linearizations of these equations around an absolute thermal equilibrium (which possesses no gradients or flows) of density $n_{0}=\bar{n}$ and temperature $T_{0}$ are

$$
\begin{gathered}
\frac{\partial}{\partial t}\left(\frac{\Delta n}{n_{0}}\right)+\nabla \cdot \Delta \boldsymbol{u}=0, \\
n_{0} m \frac{\partial \Delta \boldsymbol{u}}{\partial t}=n_{0} q \Delta \boldsymbol{E}-\nabla \Delta p-\nabla \cdot \Delta \boldsymbol{\pi}, \\
\frac{3}{2} n_{0} \frac{\partial \Delta T}{\partial t}=-p_{0} \nabla \cdot \Delta \boldsymbol{u}-\nabla \cdot \Delta \boldsymbol{q},
\end{gathered}
$$

\footnotetext{
${ }^{9}$ The inclusion of the mean density $\bar{n}$ in the definition $(2.2 a)$ of density is a normalization convention that makes $f \mathrm{~d} \boldsymbol{v}$ dimensionless. Specifically, $f$ is normalized such that $V^{-1} \int \mathrm{d} \boldsymbol{x} \mathrm{d} \boldsymbol{v} f(\boldsymbol{x}, \boldsymbol{v}, t)=1$, where $V$ is the volume of the system. Thus, $f$ differs, though inessentially, from a true probability density function (PDF), whose normalization would not include the $V^{-1}$ factor. An example of an $f$ normalized with this convention is the local Maxwellian given in (A 6).

In the general case of arbitrarily coupled plasma, there are potential-energy corrections to (2.2c). A kinetic theory for such a system was derived by Forster \& Martin (1970). One of the few deficiencies of Braginskii's review article is that he does not emphasize the restriction to a nearly ideal gas. The formalism used in Part 2 allows for arbitrarily strong coupling.

${ }^{10}$ In context, there should be no confusion between the kinematic viscosity and the mass ratio $m_{e} / m_{i}$, which I also denote by $\mu$. The distinction is clearer in multispecies plasma, where the viscosities carry species subscripts. I also use $\mu$ for the reduced mass $\mu_{s s^{\prime}}$ as well as for a contravariant or covariant index of a hydrodynamic vector.
} 
where $\Delta p \doteq T_{0} \Delta n+n_{0} \Delta T$ and

$$
\begin{gathered}
\Delta \boldsymbol{\pi} \doteq-n_{0} m \mu_{0}\left((\boldsymbol{\nabla} \Delta \boldsymbol{u})+(\boldsymbol{\nabla} \Delta \boldsymbol{u})^{\mathrm{T}}-\frac{2}{3}(\boldsymbol{\nabla} \cdot \Delta \boldsymbol{u}) \boldsymbol{l}\right), \\
\Delta \boldsymbol{q} \doteq-n_{0} \kappa_{0} \nabla \Delta T .
\end{gathered}
$$

Specific formulas for the transport coefficients $\mu_{0}$ and $\kappa_{0}$ are available (appendix A). The immediate goal is to rederive these results from the projection-operator formalism.

The starting point is the nonlinear kinetic equation

$$
\frac{\mathrm{D} f}{\mathrm{D} t} \doteq \frac{\partial f}{\partial t}+\boldsymbol{v} \cdot \nabla f+\boldsymbol{E} \cdot \partial f=-\mathrm{C}[f],
$$

where $\partial \doteq(q / m) \partial / \partial \boldsymbol{v}$ and $\mathrm{C}[f]$ is the collision operator, ${ }^{11}$ written here as a (spatially local) nonlinear functional (denoted by the square brackets) of $f$. For present purposes, one may think of $C$ as the nonlinear Landau operator. However, although use of the Landau kinetic equation is commonplace, it requires a non-trivial justification. It appears to be adequate for the treatment of first-order and linear response in a weakly coupled plasma with a statistically homogeneous background; more generally, however, it is unclear that a spatially local collision operator even exists. Further explication of this point is relegated to Part 2, as it lies well outside of the scope of this introductory discussion.

The electric field $\boldsymbol{E}=-\nabla \phi$ (I consider only the electrostatic approximation) is obtained from Poisson's equation

$$
-\nabla^{2} \phi(x, t)=4 \pi \rho=4 \pi \sum_{\bar{s}}(\bar{n} q)_{\bar{s}} \int \mathrm{d} \overline{\boldsymbol{v}} f_{\bar{s}}(\boldsymbol{x}, \overline{\boldsymbol{v}}, t) .
$$

For the OCP, one allows perturbations to only one species, so a species label is dropped in this section; the other species merely serve to provide an overall chargeneutral background. A stationary and stable solution of the kinetic-equation-Poisson system is the absolute Maxwellian,

$$
f_{\mathrm{M}}(\boldsymbol{v}) \doteq\left(2 \pi v_{\mathrm{t}}^{2}\right)^{-3 / 2} \mathrm{e}^{-v^{2} / 2 v_{\mathrm{t}}^{2}},
$$

where $^{12} v_{\mathrm{t}} \doteq(T / m)^{1 / 2}$. Infinitesimal perturbations to that equilibrium obey

$$
\partial_{t} \Delta f+\boldsymbol{v} \cdot \nabla \Delta f+\Delta \boldsymbol{E} \cdot \partial f_{\mathrm{M}}=-\widehat{\mathrm{C}} \Delta f,
$$

where $\widehat{\mathrm{C}}$ is the linearized Landau operator (discussed in appendix B). This is the basic dynamical equation used in this article. (I shall add a constant background magnetic field in §3.) As a linear dynamical evolution equation, it is analogous to the Schrödinger equation of quantum mechanics. There are, of course, important differences: the Schrödinger equation is time reversible whereas the present equation is time irreversible due to the presence of $\widehat{\mathrm{C}}$, and the quantum-mechanical wave function is a probability amplitude (whose squared modulus is a probability) whereas $f$ is directly an actual probability (density).

\footnotetext{
${ }^{11}$ The unconventional choice of minus sign on the right-hand side of (2.9) is made so that the collision operator is effectively positive, consistent with the usual convention of a positive collision frequency. The linearized operator is positive-semidefinite; see appendix B.

${ }^{12}$ Here and subsequently, I have dropped the 0 subscript on $T_{0}$. This is partly to unclutter the notation; however, there are also more significant reasons. First, it makes no difference whether coefficients of a linearresponse $(\Delta)$ quantity are evaluated with $T$ or $T_{0}$ (I only consider linear response in the present article). Second, when one considers the theory of transport beyond linear order, one finds that coefficients are actually evaluated with the total (local) temperature $T(x, t)$. Thus, formulas written in terms of $T$ rather than $T_{0}$ will readily generalize to the more complete second-order theory discussed in Part 2.
} 


\subsubsection{Definition of the projection operator}

In this and the next several sections I shall use the Schrödinger representation, in which averages of time-independent functions of velocity are taken with the time evolved $f(t)$, which plays the role of a time-dependent state vector. It is useful to treat those averages as projections, and it is convenient to realize those projections by using a time-independent scalar product. I shall use a Dirac bra-ket notation with a hidden weight function $f_{\mathrm{M}}$. Thus, for any functions $A(\boldsymbol{v})$ and $B(\boldsymbol{v})$,

$$
\begin{gathered}
\langle A| \doteq A(\boldsymbol{v}), \\
|A\rangle \doteq A(\boldsymbol{v}) f_{\mathrm{M}}(\boldsymbol{v}), \\
\langle A|\mathrm{~L}| B\rangle \doteq \int \mathrm{d} \boldsymbol{v} \mathrm{d} \overline{\boldsymbol{v}} A(\boldsymbol{v}) L(\boldsymbol{v}, \overline{\boldsymbol{v}}) B(\overline{\boldsymbol{v}}) f_{\mathrm{M}}(\overline{\boldsymbol{v}}),
\end{gathered}
$$

where $\mathrm{L}$ is a linear operator with two-point kernel ${ }^{13} L\left(\boldsymbol{v}, \boldsymbol{v}^{\prime}\right)$. When multispecies plasmas are discussed later, the scalar product will be extended to include species summations. Note that no complex conjugate appears in this definition, unlike the analogous situation in quantum mechanics. This scalar product is 'natural' because (appendix B) $\bar{n} \widehat{C}$ is self-adjoint with respect to it:

$$
\langle A|\bar{n} \widehat{\mathrm{C}}| B\rangle=\langle B|\bar{n} \widehat{\mathrm{C}}| A\rangle .
$$

A special case of $(2.13 c)$ is obtained by specializing $\mathrm{L}$ to the identity operator $\mathrm{I} \rightarrow$ $\delta(\boldsymbol{v}-\overline{\boldsymbol{v}})$ :

$$
\langle A \mid B\rangle=\int \mathrm{d} \boldsymbol{v} A(\boldsymbol{v}) B(\boldsymbol{v}) f_{\mathrm{M}}(\boldsymbol{v}),
$$

just the equilibrium velocity average of the product $A B:\langle A \mid B\rangle=\langle A B\rangle_{\mathrm{M}}$. I shall often drop the M subscript.

Note that the scalar product used in this paper is defined on just the velocity space. That this is useful is a consequence of the assumptions that the collision operator is spatially local and that no potential-energy contributions are included in the definition of the hydrodynamic moments (see footnote 9 on page 9 and the discussion below). In Part 2, I shall remove those restrictions and employ a more general scalar product that generalizes the present one to also involve spatial integration.

Even for spatially local $\widehat{\mathrm{C}}$, other operators as well as the bras and kets will in general depend on space. The spatial dependence will often be removed by Fourier transformation, so constructions such as $\langle A \mid B\rangle$ will be labelled by an (often implicit) $\boldsymbol{x}$ or $\boldsymbol{k}^{14}$

If one writes $\Delta f \doteq \Delta \chi f_{\mathrm{M}}$ for unknown (dimensionless) $\Delta \chi,(2.12)$ becomes

$$
\partial_{t}|\Delta \chi\rangle+\boldsymbol{v} \cdot \nabla|\Delta \chi\rangle+\left|\partial \ln f_{\mathrm{M}}\right\rangle \cdot \Delta \boldsymbol{E}=-\widehat{\mathrm{C}}|\Delta \chi\rangle,
$$

where $\left|\boldsymbol{\partial} \ln f_{\mathrm{M}}\right\rangle=-(q / T)|\boldsymbol{v}\rangle$. In collisional transport theory, the conservation properties of the collision operator are crucial. For the Landau operator, those are ${ }^{15}$

$$
\langle\bar{n} \breve{A}| \widehat{C}=0,
$$

\footnotetext{
${ }^{13}$ For example, if $\mathrm{L} \doteq \partial / \partial \boldsymbol{v}$, then $L\left(\boldsymbol{v}, \boldsymbol{v}^{\prime}\right)=\partial_{\boldsymbol{v}} \delta\left(\boldsymbol{v}-\boldsymbol{v}^{\prime}\right)$. Here 'kernel' is used in one of its two standard meanings; it does not refer here to the null space of the operator.

${ }^{14}$ For example, we shall encounter the operator $\mathrm{L}_{\boldsymbol{v}} \doteq-\mathrm{i} \boldsymbol{v} \cdot \boldsymbol{\nabla}$. For use with the scalar product, the kernel of this operator is $L_{\boldsymbol{v}}\left(\boldsymbol{v}, \boldsymbol{v}^{\prime}\right)=-\mathrm{i}\left[\boldsymbol{v} \delta\left(\boldsymbol{v}-\boldsymbol{v}^{\prime}\right)\right] \cdot \boldsymbol{\nabla} \rightarrow \boldsymbol{k} \cdot \boldsymbol{v} \delta\left(\boldsymbol{v}-\boldsymbol{v}^{\prime}\right)$.

${ }^{15}$ In (2.14) and (2.17), the $\bar{n}$ is unnecessary, as it is a constant. It is retained so that the formula looks identical to that for multispecies plasma $(\S 3)$, where the scalar product includes a species summation.
} 
where $\bar{n}$ is the spatially constant density of the equilibrium,

$$
\breve{\boldsymbol{A}} \doteq\left(1 \boldsymbol{P}^{\mathrm{T}} K\right)^{\mathrm{T}},
$$

$\boldsymbol{P} \doteq m \boldsymbol{v}, K \doteq(1 / 2) m v^{2}$, and the breve accent is used to distinguish non-orthogonal functions from more convenient orthogonal ones to be introduced shortly. ${ }^{16}$ Thus, $\langle\breve{\boldsymbol{A}}|$ are the five null left eigenfunctions of $\bar{n} \widehat{\mathrm{C}}$. (In fact, there is no need to distinguish left and right eigenfunctions because for the Landau operator $\bar{n} \widehat{\mathrm{C}}$ is self-adjoint with respect to the chosen scalar product; see $\S$ B.1.) Those eigenfunctions span a preferred five-dimensional hydrodynamic subspace $\mathcal{H}$ (see figure 1 ).

Clearly the definition of $\mathcal{H}$ is tied to the form of the collision operator that is assumed. A consequence of assuming the Landau operator (appropriate for weak coupling) is that no potential-energy contributions appear in the eigenfunctions. Thus, the present $\mathcal{H}$ is not the true hydrodynamic subspace of the many-particle system. This approximation is relaxed in Part 2.

From the definitions of $n, \boldsymbol{u}$ and $T$, it follows that

$$
\left\langle 1 \mid f / f_{\mathrm{M}}\right\rangle=n / \bar{n},
$$

so

$$
\langle 1 \mid \Delta \chi\rangle=\Delta n / \bar{n}
$$

Similarly,

$$
\langle\boldsymbol{v} \mid \Delta \chi\rangle=\Delta \boldsymbol{u}
$$

(the absolute equilibrium has no flow). Finally,

$$
\langle K \mid \Delta \chi\rangle=\frac{3}{2} \Delta T+\frac{3}{2} T\left(\frac{\Delta n}{\bar{n}}\right) .
$$

Thus, the perturbed (kinetic) temperature can be extracted by

$$
\frac{3}{2} \Delta T=\left\langle K^{\prime} \mid \Delta \chi\right\rangle
$$

where

$$
K^{\prime} \doteq K-\langle K\rangle=\frac{1}{2} m v^{2}-\frac{3}{2} T .
$$

This motivates the introduction of

$$
\boldsymbol{A} \doteq\left(\begin{array}{lll}
1 & \boldsymbol{P}^{\mathrm{T}} & K^{\prime}
\end{array}\right)^{\mathrm{T}},
$$

where $\boldsymbol{P}^{\prime}=\boldsymbol{P}-\langle\boldsymbol{P}\rangle=\boldsymbol{P}$; with this definition, the components of $\boldsymbol{A}$ are orthogonal. Then the deviations of the statistically averaged hydrodynamic variables from their equilibrium values are given by

$$
\Delta \boldsymbol{a} \doteq\langle\boldsymbol{A} \mid \Delta \chi\rangle=\left(\frac{\Delta n}{\bar{n}} \Delta \boldsymbol{p}^{\mathrm{T}} \frac{3}{2} \Delta T\right)^{\mathrm{T}}
$$

where $\Delta \boldsymbol{p} \doteq m \Delta \boldsymbol{u}$.

\footnotetext{
${ }^{16}$ The choice of upper case for the velocity-dependent functions $\boldsymbol{P}$ and $K$ is made for consistency with the convention of Brey et al. (1981), whose approach I shall discuss in Part 2. Another notational possibility would have been to use a tilde, a notation I have often used in other articles to denote a random variable.
} 
These results lead one to define the hydrodynamic projection operator as

$$
\mathrm{P} \doteq\left|\boldsymbol{A}^{\mathrm{T}}\right\rangle \cdot \boldsymbol{M}^{-1} \cdot\langle\boldsymbol{A}|,
$$

where the normalization matrix ${ }^{17}$

$$
\boldsymbol{M} \doteq\left\langle\boldsymbol{A} \boldsymbol{A}^{\mathrm{T}}\right\rangle=\left(\begin{array}{ccc}
1 & \mathbf{0}^{\mathrm{T}} & 0 \\
\mathbf{0} & N_{\boldsymbol{p}} \boldsymbol{I} & \mathbf{0} \\
0 & \mathbf{0}^{\mathrm{T}} & N_{T}
\end{array}\right)
$$

ensures that $\mathrm{P}^{2}=\mathrm{P}$; one easily calculates that

$$
N_{p} \doteq\left(m v_{\mathrm{t}}\right)^{2}, \quad N_{T} \doteq \frac{3}{2} T^{2} .
$$

The projection of the state vector is thus

$$
\mathrm{P}|\Delta \chi\rangle=\left|\boldsymbol{A}^{\mathrm{T}}\right\rangle \cdot \boldsymbol{M}^{-1} \cdot\langle\boldsymbol{A} \mid \Delta \chi\rangle=\left|\boldsymbol{A}^{\mathrm{T}}\right\rangle \cdot \boldsymbol{M}^{-1} \cdot \Delta \boldsymbol{a}
$$

and the hydrodynamic variables can be extracted by taking the scalar product of $\boldsymbol{A}$ with that projection:

$$
\Delta \boldsymbol{a}(t)=\langle\boldsymbol{A}|\mathrm{P}| \Delta \chi(t)\rangle .
$$

The projection formalism can be couched in a manifestly covariant fashion that turns out to be very convenient. Namely, if the components of $\boldsymbol{A}$ are labelled as $A^{\mu}$, where $\mu=1, \ldots, 5$ (or $\mu \in\{n, \boldsymbol{p}, T\}$ ), and if $\left(\boldsymbol{M}^{-1}\right)_{\mu \nu}$ is treated as a metric tensor $g_{\mu \nu}$ that lowers indices according to $A_{\mu}=g_{\mu \nu} A^{\nu}$ (where Einstein's convention for summation over repeated indices is adopted), then $\mathrm{P}$ can be written as

$$
\mathrm{P}=\left|A_{\mu}\right\rangle\left\langle A^{\mu}\right|
$$

One has

$$
\left\langle A^{\mu} \mid A_{\mu^{\prime}}\right\rangle=\delta_{\mu^{\prime}}^{\mu}
$$

the hydrodynamic projection of the state vector is

$$
\mathrm{P}|\Delta \chi(t)\rangle=\left|A_{\mu}\right\rangle \Delta a^{\mu}(t)
$$

(cf. (2.30)), and the hydrodynamic variables themselves are

$$
\Delta a^{\mu}(t)=\left\langle A^{\mu} \mid \Delta \chi(t)\right\rangle=\left\langle A^{\mu}|\mathrm{P}| \Delta \chi(t)\right\rangle
$$

(cf. (2.26) and (2.31)). This representation, which identifies the $\Delta a^{\mu}$ values as the contravariant components of a hydrodynamic vector, is further discussed in appendix $\mathrm{C}$.

${ }^{17}$ With an appropriate redefinition of $\boldsymbol{A}, \boldsymbol{M}$ could be made the identity matrix. However, that is not convenient for obtaining the conventional fluid perturbations according to (2.26), and it does not distinguish between contravariant and covariant components (see appendix C). Moreover, it turns out that such a redefinition does not naturally generalize to the formalism used in Part 2 for treating second-order effects. 


\subsubsection{Projecting the kinetic equation; the statistical closure problem}

Because $\mathrm{P}$ is a time-independent linear operator, one can extract the linearized fluid moment equations by applying $\mathrm{P}$ to $(2.16)$, or equivalently by taking the time derivative of (2.31). Define

$$
\mathcal{L} \doteq \mathrm{L}+\mathrm{L}_{E} \quad \text { and } \quad \mathrm{L} \doteq \mathrm{L}_{v}+\mathrm{L}_{\mathrm{C}}
$$

where

$$
\mathrm{iL}_{\boldsymbol{v}} \doteq \boldsymbol{v} \cdot \nabla, \quad \mathrm{iL}_{\mathrm{C}} \doteq \widehat{\mathrm{C}}, \quad \mathrm{iL}_{E} \doteq-(q / T)|\boldsymbol{v}\rangle \cdot\langle\mathbb{E}|,
$$

and $\mathbb{E}$ is the linear operator that solves Poisson's equation for $\Delta \boldsymbol{E}$ in terms of $\Delta \chi$ according to $\Delta \boldsymbol{E}=\langle\mathbb{E} \mid \Delta \chi\rangle$; in Fourier space, the kernel of $\mathbb{E}$ is

$$
\mathbb{E}_{k}\left(\boldsymbol{v}, \boldsymbol{v}^{\prime}\right)=\boldsymbol{\epsilon}_{k} \bar{n} q, \quad \boldsymbol{\epsilon}_{k} \doteq-4 \pi \mathrm{i} \boldsymbol{k} / k^{2}
$$

$\left(\boldsymbol{\epsilon}_{k}\right.$ is the Fourier transform of the electric field of a unit point charge). Then the hydrodynamic projection of the linearized kinetic equation is

$$
\partial_{t} \mathrm{P}|\Delta \chi\rangle+\mathrm{Pi} \mathcal{L}|\Delta \chi\rangle=0 .
$$

The ultimate goal is to obtain a closed system for $\mathrm{P}|\Delta \chi\rangle$; that is called 'solving the closure problem'. That would be accomplished if one could move the $\mathrm{P}$ past the $\mathcal{L}$ (i.e. if $\mathrm{P}$ and $\mathcal{L}$ commute $-[\mathrm{P}, \mathcal{L}] \doteq \mathrm{P} \mathcal{L}-\mathcal{L} \mathrm{P}=0$ ). However, this is not entirely possible. From (2.37), one sees that the $\Delta \boldsymbol{E}$ term is proportional to $|\boldsymbol{v}\rangle$ and thus, given the definition (2.27) of $\mathrm{P}$, lies entirely in the hydrodynamic subspace [which is why $\mathrm{L}_{E}$ was broken out separately in (2.36)]. A consequence is that $\mathrm{P}$ and $\mathrm{L}_{E}$ commute, so there is no closure issue stemming from $\mathrm{L}_{\boldsymbol{E}}$. However, the PiL term is problematical because $\mathrm{P}$ and $\mathrm{L} \doteq \mathrm{L}_{v}+\mathrm{L}_{\mathrm{C}}$ do not commute. Although for the OCP it is true that $[\mathrm{P}, \widehat{\mathrm{C}}]=0$ as a consequence of the conservation laws, that is not true in general, ${ }^{18}$ and it is never the case that $[\mathrm{P}, \boldsymbol{v}]=0$. In order to see this, note that while $\mathrm{P} \boldsymbol{v} \propto|\boldsymbol{A}\rangle$ lies in the hydrodynamic subspace by construction, one has

$$
\boldsymbol{v} \mathrm{P} \propto|\boldsymbol{v} \boldsymbol{A}\rangle=\left(\begin{array}{c}
|\boldsymbol{v}\rangle \\
|\boldsymbol{v}(m \boldsymbol{v})\rangle \\
\left|\boldsymbol{v}\left(\frac{1}{2} m v^{2}-\frac{3}{2} T\right)\right\rangle
\end{array}\right) .
$$

While the density component of this vector lies in the hydrodynamic subspace, as do the diagonal elements of the momentum component $\left[\boldsymbol{v} v=(1 / 3) v^{2} \boldsymbol{I}+\left(\boldsymbol{v} \boldsymbol{v}-(1 / 3) v^{2} \boldsymbol{I}\right)\right]$ and the (3/2)|v $\rangle T$ term, the off-diagonal terms and the kinetic-energy flux $\left|(1 / 2) m v^{2} \boldsymbol{v}\right\rangle$ lie in the orthogonal subspace. This is the familiar problem of moment closure, formalized here in terms of projection operators. It is one instance of the famous statistical closure problem much discussed in analytical turbulence theory (Krommes 2002, 2015, and references therein), here involving averages over a random velocity variable.

To deal with this difficulty, I follow Mori and insert the identity $P+Q=1$ so that

$$
\mathrm{P} \mathcal{L}|\Delta \chi\rangle=\mathrm{P} \mathcal{L}(\mathrm{P}+\mathrm{Q})|\Delta \chi\rangle=\mathrm{P} \mathcal{L} \mathrm{P}|\mathrm{P} \Delta \chi\rangle+\mathrm{P} \mathcal{L} \mathrm{Q}|\mathrm{Q} \Delta \chi\rangle .
$$

\footnotetext{
${ }^{18}$ For the multispecies case discussed in $\S 3,[\mathrm{P}, \widehat{\mathrm{C}}] \neq 0$ since $\mathrm{P}$ is chosen to project into a species-dependent subspace but the conservation laws involve species summation.
} 
(In writing the last form, I used $\mathrm{P}^{2}=\mathrm{P}$ and $\mathrm{Q}^{2}=\mathrm{Q}$. I also slightly abused the notation ${ }^{19}$ to write $\mathrm{P}|\Delta \chi\rangle \equiv|\mathrm{P} \Delta \chi\rangle$.) Let us define the frequency matrix $\boldsymbol{\Omega}$ as

$$
\boldsymbol{\Omega} \doteq\left\langle\boldsymbol{A}|\mathcal{L}| \boldsymbol{A}^{\mathrm{T}}\right\rangle \cdot \boldsymbol{M}^{-1} ;
$$

this is a normalized version of the hydrodynamic matrix element of $\mathrm{P} \mathcal{L} \mathrm{P}$. The nomenclature is consistent with the fact that under Fourier transformation $v \cdot \nabla \rightarrow$ $\mathrm{i} \boldsymbol{k} \cdot \boldsymbol{v}$, which corresponds to the streaming frequency $\Omega_{\boldsymbol{v}} \doteq \boldsymbol{k} \cdot \boldsymbol{v}$. In general, the 'frequency' is complex because $\mathcal{L}$ involves the dissipative term $\mathrm{L}_{\mathrm{C}} \doteq-\mathrm{i} \widehat{\mathrm{C}}$. However, for the OCP $\widehat{\mathrm{C}}$ does not contribute to the frequency matrix since $\mathrm{PC}=0$ as a consequence of the conservation laws. In any event, if the $\mathrm{P} \mathcal{L Q}$ term in (2.41) were to vanish, then one would have a matrix equation for $|\mathrm{P} \Delta \chi\rangle$ (or for the $\Delta \boldsymbol{a}$ values) and the closure problem would be solved. Unfortunately, this is not the case because, as shown above, $[\mathrm{P}, \boldsymbol{v}] \neq \mathbf{0}$. (This is one way of stating that the kinetic equation is stochastically nonlinear in the velocity variable.) Thus, the dynamics in the hydrodynamic subspace,

$$
\partial_{t}|\mathrm{P} \Delta \chi\rangle+\left|\boldsymbol{A}^{\mathrm{T}}\right\rangle \cdot \boldsymbol{M}^{-1} \cdot \mathrm{i} \boldsymbol{\Omega} \cdot\langle\boldsymbol{A} \mid \mathrm{P} \Delta \chi\rangle+\mathrm{PiLQ}|\mathrm{Q} \Delta \chi\rangle=0
$$

or, upon applying $\langle\boldsymbol{A}|$ to (2.43),

$$
\partial_{t} \Delta \boldsymbol{a}+\mathrm{i} \boldsymbol{\Omega} \cdot \Delta \boldsymbol{a}+\langle\boldsymbol{A}|\mathrm{iLQ}| \mathrm{Q} \Delta \chi\rangle=0,
$$

is coupled to that in the orthogonal subspace. Note that only $\mathrm{L}\left(\mathcal{L}\right.$ sans $\left.\mathrm{L}_{E}\right)$ enters the last term of (2.44); $\mathrm{QL}_{E}=\mathrm{L}_{E} \mathrm{Q}=0$ since $\mathrm{L}_{E} \propto|\boldsymbol{v}\rangle\langle 1|$ has components only in $\mathcal{H}$.

In order to obtain a closed system, it is necessary to eliminate $|\mathrm{Q} \Delta \chi\rangle$ in favour of some function of $\Delta \boldsymbol{a}$. Upon applying Q to (2.16), one obtains

$$
\partial_{t}|\mathrm{Q} \Delta \chi\rangle+\mathrm{QiLQ}|\mathrm{Q} \Delta \chi\rangle+\mathrm{QiLP}|\mathrm{P} \Delta \chi\rangle=0 .
$$

Since QiLQ is a linear operator, equation (2.45) can be solved by means of a Green's function:

$$
|\mathrm{Q} \Delta \chi\rangle(t)=\mathrm{G}_{\mathrm{Q}}(t ; 0)|\mathrm{Q} \Delta \chi(0)\rangle-\int_{0}^{t} \mathrm{~d} \bar{t} \mathrm{G}_{\mathrm{Q}}(t ; \bar{t}) \mathrm{QiLP}|\mathrm{P} \Delta \chi(\bar{t})\rangle,
$$

where

$$
\mathrm{G}_{\mathrm{Q}}\left(t ; t^{\prime}\right) \doteq H\left(t-t^{\prime}\right) \exp \left[-\mathrm{QiLQ}\left(t-t^{\prime}\right)\right]
$$

(since $\mathrm{Q}$ and $\mathrm{L}$ are time independent). Here $H(\tau)$ is the unit step function. ${ }^{20}$ Only the time arguments are written explicitly in the previous two equations, although in reality the kernels of two-point operators such as $\mathrm{G}_{\mathrm{Q}}$ also depend on two space and two velocity variables, so convolutions over those variables are implied. Because the background is spatially homogeneous and the equations are linear, one can Fourier transform in space. (This is not necessary, but it makes some of the notation less cumbersome.) It is conventional to ignore the initial condition $|\mathrm{Q} \Delta \chi(0)\rangle$. Physically,

\footnotetext{
${ }^{19}$ Strictly speaking, the $\mathrm{P}$ should remain outside of the ket because it operates on the hidden $f_{\mathrm{M}}$ as well as on $\Delta \chi$.

${ }^{20} H(\tau)=0$ if $\tau<0,1 / 2$ if $\tau=0$, or 1 if $\tau>0$.
} 
this means that the system is prepared to lie entirely in the hydrodynamic subspace. Then one obtains

$$
\partial_{t} \Delta \boldsymbol{a}_{k}(t)+\mathrm{i} \boldsymbol{\Omega}_{\boldsymbol{k}} \cdot \Delta \boldsymbol{a}_{\boldsymbol{k}}(t)+\int_{0}^{t} \mathrm{~d} \bar{\tau} \boldsymbol{\Sigma}_{\boldsymbol{k}}(\bar{\tau}) \cdot \Delta \boldsymbol{a}_{\boldsymbol{k}}(t-\bar{\tau})=0,
$$

where $^{21}$

$$
\boldsymbol{\Sigma}_{\boldsymbol{k}}(\tau) \doteq\left\langle\boldsymbol{A}\left|\mathrm{L}_{\boldsymbol{k}} \mathrm{QG}_{\mathrm{Q}, \boldsymbol{k}}(\tau) \mathrm{QL}_{\boldsymbol{k}}\right| \boldsymbol{A}^{\mathrm{T}}\right\rangle \cdot \boldsymbol{M}^{-1}
$$

Although (2.48) is now closed in terms of $\Delta \boldsymbol{a}$, that system does not yet have the form of conventional linearized fluid equations because it is non-local in time. To obtain the conventional form, one makes a Markovian approximation. In the time domain, that means that one assumes that $\Delta \boldsymbol{a}_{k}(t)$ varies much more slowly than the characteristic decay time of $\boldsymbol{\Sigma}_{\boldsymbol{k}}(\tau)$. Then (ignoring spatial or wavenumber dependence for the moment)

$$
\int_{0}^{t} \mathrm{~d} \bar{\tau} \boldsymbol{\Sigma}(\bar{\tau}) \cdot \Delta \boldsymbol{a}(t-\bar{\tau}) \approx\left(\int_{0}^{t} \mathrm{~d} \bar{\tau} \boldsymbol{\Sigma}(\bar{\tau})\right) \cdot \Delta \boldsymbol{a}(t) \approx\left(\int_{0}^{\infty} \mathrm{d} \bar{\tau} \boldsymbol{\Sigma}(\bar{\tau})\right) \cdot \Delta \boldsymbol{a}(t) .
$$

The temporal non-locality can also be represented in frequency space. The one-sided temporal Fourier transform of (2.48) (denoted by a hat) is

$$
\left[-\mathrm{i} \omega \boldsymbol{I}+\mathrm{i} \boldsymbol{\Omega}_{\boldsymbol{k}}+\widehat{\boldsymbol{\Sigma}}_{\boldsymbol{k}}(\omega)\right] \cdot \Delta \widehat{\boldsymbol{a}}_{\boldsymbol{k}}(\omega)=\Delta \boldsymbol{a}_{\boldsymbol{k}}(0) .
$$

The temporal Markovian approximation in (2.50) becomes $\widehat{\boldsymbol{\Sigma}}(\omega) \approx \widehat{\boldsymbol{\Sigma}}(0)$.

Because $\boldsymbol{\Sigma}_{\boldsymbol{k}}(\tau)$ depends on $\boldsymbol{k}$, the inverse spatial Fourier transform of (2.48) becomes a convolution in space and thus demonstrates several kinds of spatial non-locality - one from the $\mathrm{L}_{k}$ operators, and one from $\mathrm{G}_{\mathrm{Q}, k}$. The contribution from the $\mathrm{L}_{k}$ operators is easy to understand. For the OCP, it is straightforward to see that only $\mathrm{L}_{\boldsymbol{v}, \boldsymbol{k}} \doteq \boldsymbol{k} \cdot \boldsymbol{v}$ contributes to (2.49) because the components of $\boldsymbol{A}$ are the null eigenvectors of $\widehat{\mathrm{C}}$. That means that $\boldsymbol{\Sigma}_{\boldsymbol{k}}(\tau) \propto k^{2}$. This is the Fourier representation of a diffusion process, corresponding to the spatial operator $-\nabla^{2}$, and is the expected result for a conventional transport theory. $\left(\nabla^{2}\right.$ is non-local because it couples neighbouring points in space.)

Remaining to discuss is the $\boldsymbol{k}$ dependence of $\mathrm{G}_{\mathrm{Q}, \boldsymbol{k}}$. Completely analogous to the temporal Markovian approximation described above, conventional collisional transport theory will emerge if one can make the spatial Markovian approximation $\mathrm{G}_{\mathrm{Q}, k} \approx \mathrm{G}_{\mathrm{Q}, 0}$.

To see whether these Markovian approximations are possible, let us look more closely at $\boldsymbol{\Sigma}_{\boldsymbol{k}}(\tau)$. For scale lengths that are long compared to the mean free path, one can approximate $\exp [-\mathrm{Q}(\boldsymbol{v} \cdot \boldsymbol{\nabla}+\widehat{\mathrm{C}}) \mathrm{Q} \tau] \rightarrow \exp [-\mathrm{Q}(\mathrm{i} \boldsymbol{k} \cdot \boldsymbol{v}+\widehat{\mathrm{C}}) \mathrm{Q} \tau] \approx \exp (-\mathrm{Q} \widehat{\mathrm{C}} \mathrm{Q} \tau)$. (This $k \rightarrow 0$ limit is the spatial Markovian approximation.) For the OCP, one has $\widehat{\mathrm{PC}}=\widehat{\mathrm{CP}}=0$, so $\exp (-\mathrm{Q} \widehat{\mathrm{C}} \mathrm{Q} \tau) \rightarrow \exp (-\widehat{\mathrm{C}} \tau)$. If this operator were to act on an element of $\mathcal{H}$, which contains the null eigenfunctions of $\widehat{\mathrm{C}}$, it would not decay and one would be in grave difficulty. However, in $\boldsymbol{\Sigma}_{\boldsymbol{k}}(\tau)$ this exponential is sandwiched between two $\mathrm{Q}$ operators, giving the construction $\mathrm{Q} \exp (-\widehat{\mathrm{C}} \tau) \mathrm{Q}$. Those $\mathrm{Q}$ operators 'protect' $\widehat{\mathrm{C}}$ from the null space. Thus, the null eigenvalues of $\widehat{\mathrm{C}}$ do not contribute

${ }^{21}$ Elementary discussion of the role of the 'mass operator' $\boldsymbol{\Sigma}$ in more general contexts is given by Krommes (2015). More advanced details can be found in Krommes (2002). 
and the effect of $\widehat{\mathrm{C}}$ is roughly to provide a damping $\mathrm{e}^{-\lambda \tau}$, where $\lambda$ is of the order of the collision frequency $v$. (For discussion and examples of this assertion, which for the Landau operator is non-trivial, see $\S$ B.4.) Thus, since $\boldsymbol{\Sigma}(\tau)$ has a finite decay time $\sim v^{-1}$, the temporal Markovian approximation is justifiable and we have been led to a conventional set of time-local (Markovian) fluid equations:

$$
\partial_{t} \Delta a_{k}(t)+\left(\mathrm{i} \Omega_{k}+\eta_{k}\right) \cdot \Delta a_{k}(t)=0,
$$

where (for generality and later application to multispecies plasmas I reinstate the original construction $\exp (-\mathrm{Q} \widehat{\mathrm{C}} \mathrm{Q} \tau))$

$$
\eta_{k} \doteq \int_{0}^{\infty} \mathrm{d} \tau \boldsymbol{\Sigma}_{k}(\tau) \approx\left\langle\boldsymbol{A}\left|\mathrm{L}_{k} \mathrm{Q}(\mathrm{QCQ})^{-1} \mathrm{QL}_{k}\right| \boldsymbol{A}^{\mathrm{T}}\right\rangle \cdot \boldsymbol{M}^{-1},
$$

or covariantly

$$
\boldsymbol{\eta}_{\nu, \boldsymbol{k}}^{\mu}=\left\langle A^{\mu}\left|\mathrm{L}_{\boldsymbol{k}} \mathrm{Q}(\mathrm{QCQ})^{-1} \mathrm{QL}_{\boldsymbol{k}}\right| A_{\nu}\right\rangle .
$$

The result just obtained represents the lowest-order term in the Taylor expansion of $\Delta \boldsymbol{a}(\bar{\tau})$ in (2.50). In principle, one could carry that expansion to higher order; at second order, one would ultimately obtain linear Burnett effects. However, I shall defer such a calculation to Part 2 .

To reemphasize and reiterate the role of the $\mathrm{Q}$ operators, I return to the formal expression for $\boldsymbol{\eta}$, which involves the inverse of the operator Q $\widehat{C} Q$ applied to a quantity in the orthogonal subspace $O$. It is important to understand the sense in which this inverse exists. A quantity of the form $|\psi\rangle \doteq(\mathrm{Q} \widehat{\mathrm{CQ}})^{-1} \mathrm{Q}|\Delta \chi\rangle$ can be obtained as the solution to the equation ( $\widehat{Q} \widehat{C Q})|\psi\rangle=\mathrm{Q}|\Delta \chi\rangle$. For this equation to have a solution, the source term on the right-hand side must be orthogonal to the left null eigenfunctions of the operator on the left. That is true; both sides are annihilated by P. Thus, by Fredholm's alternative theorem, the equation has a solution, but it is not unique because an arbitrary superposition $\sum_{i} \alpha_{i}|i\rangle$ of the null eigenfunctions $|i\rangle$ can be added to $|\psi\rangle$ without changing the equation. However, according to (2.53) or (2.54), one requires $\mathrm{Q}|\psi\rangle$, so the $\alpha_{i}$ terms do not contribute. Thus, those terms may be set to 0 , leaving one with a unique solution.

To see that the formalism recovers the conventional linearized fluid equations for the OCP, one needs to work out the various matrix elements. For variety, I shall here use the spatial form of the operators, but the discussion could equally well be conducted in $\boldsymbol{k}$ space. Let us begin with the frequency matrix:

$$
\mathrm{i} \boldsymbol{\Omega}=\mathrm{i} \boldsymbol{\Omega}_{v}+\mathrm{i} \boldsymbol{\Omega}_{\mathrm{C}}+\mathrm{i} \boldsymbol{\Omega}_{\boldsymbol{E}}
$$

where

$$
\begin{aligned}
\mathrm{i} \boldsymbol{\Omega}_{\boldsymbol{v}} & \doteq\left\langle\boldsymbol{A}|\boldsymbol{v} \cdot \boldsymbol{\nabla}| \boldsymbol{A}^{\mathrm{T}}\right\rangle \cdot \boldsymbol{M}^{-1}, \\
\mathrm{i} \boldsymbol{\Omega}_{\mathrm{C}} & \doteq\left\langle\boldsymbol{A}|\widehat{\mathrm{C}}| \boldsymbol{A}^{\mathrm{T}}\right\rangle \cdot \boldsymbol{M}^{-1}, \\
\mathrm{i} \boldsymbol{\Omega}_{\boldsymbol{E}} & \doteq\left\langle\boldsymbol{A}\left|\mathrm{iL} \mathrm{L}_{\boldsymbol{E}}\right| \boldsymbol{A}^{\mathrm{T}}\right\rangle \cdot \boldsymbol{M}^{-1} .
\end{aligned}
$$

In these expressions, the role of the $\boldsymbol{M}^{-1}$ is to change contravariant indices to covariant ones. Thus, for example,

$$
\left(\mathrm{i} \boldsymbol{\Omega}_{v}\right)_{v}^{\mu}=\left\langle A^{\mu}|\boldsymbol{v} \cdot \nabla| A_{v}\right\rangle .
$$


(Note that this mixed tensor has the dimensions of frequency.) For the OCP, $\boldsymbol{\Omega}_{\mathrm{C}}$ vanishes because $\langle\boldsymbol{A}|$ is a left null eigenfunction of $\widehat{\mathrm{C}}$. The $\boldsymbol{\Omega}_{E}$ term is readily shown to give rise to the electric-force term in the momentum equation. Finally, with $i, j$, and $k$ denoting Cartesian components of the momentum vector, one finds

$$
\left\langle\boldsymbol{A}\left|v_{k}\right| \boldsymbol{A}^{\mathrm{T}}\right\rangle=\left(\begin{array}{ccc}
0 & T \delta_{j k} & 0 \\
T \delta_{i k} & \boldsymbol{0} & T^{2} \delta_{i k} \\
0 & T^{2} \delta_{j k} & 0
\end{array}\right) .
$$

Upon multiplying on the right by $\boldsymbol{M}^{-1}$, one finds

$$
\left(\mathrm{i} \boldsymbol{\Omega}_{\boldsymbol{v}}\right)_{v}^{\mu}=\left(\begin{array}{ccc}
0 & m^{-1} \nabla_{j} & 0 \\
T \nabla_{i} & \boldsymbol{0} & \frac{2}{3} \nabla_{i} \\
0 & T m^{-1} \nabla_{j} & 0
\end{array}\right) .
$$

Note that the spatial Fourier transform of this matrix is proportional to $\boldsymbol{k}$.

To illustrate the content of $\boldsymbol{\Omega}$, consider the density component of (2.52). It is easy to see that there is no $\eta$ contribution because $\langle 1|(\boldsymbol{v} \cdot \nabla+\widehat{\mathrm{C}}) \mathrm{Q}=\boldsymbol{\nabla} \cdot\langle\boldsymbol{v}| \mathrm{Q}+\langle 1| \widehat{\mathrm{C}} \mathrm{Q}=0$. The last equality follows since $|\boldsymbol{v}\rangle$ is in the hydrodynamic subspace, which is orthogonal to $\mathrm{Q}$, and since $\widehat{\mathrm{C}}$ conserves number. But $\Omega_{p}^{n}$ couples density to momentum, and one obtains

$$
\frac{\partial}{\partial t}\left(\frac{\Delta n}{\bar{n}}\right)+\nabla \cdot \Delta \boldsymbol{u}=0
$$

which reproduces the linearized continuity equation (2.7a). As another illustration, consider the velocity component of (2.52). One readily finds that

$$
\frac{\partial \Delta \boldsymbol{p}}{\partial t}=q \Delta \boldsymbol{E}-\bar{n}^{-1} \nabla \Delta p+\cdots=0,
$$

where $\Delta p=T \Delta n+\bar{n} \Delta T$ and the centred dots denote the contribution from $\eta$, which will be discussed in the next paragraph. The explicitly written terms reproduce the correct non-dissipative (Euler) part of the linearized momentum equation (2.7b). Similarly, the temperature projection leads to

$$
\frac{3}{2} \frac{\partial \Delta T}{\partial t}=-T \nabla \cdot \Delta \boldsymbol{u}+\cdots
$$

the explicitly written terms being the Euler part of the linearized temperature equation $(2.7 c)$.

Now consider the dissipative contributions from $\eta$. I shall discuss the stress tensor in detail, leaving the heat-flow vector as an exercise for the reader. One has (now dropping the prime on $\boldsymbol{v}^{\prime}$ since there is no flow in the equilibrium, and specifically considering the $\mathrm{OCP}$ )

$$
\eta_{v}^{p}=-\left\langle m \boldsymbol{v}\left|\boldsymbol{v} \cdot \nabla \mathrm{Q} \widehat{\mathrm{C}}^{-1} \mathrm{Q} \boldsymbol{v} \cdot \nabla\right| A_{v}\right\rangle .
$$

The density component $\eta_{n}^{p}$ vanishes because $A_{n}=1$ and $\mathrm{Q}|\boldsymbol{v}\rangle=\mathbf{0}$. The temperature contribution vanishes by a symmetry argument that uses the fact that $\widehat{\mathrm{C}}$ is rotationally invariant. For the self-coupling $\eta_{p}^{p}$, note that

$$
\mathrm{Q} v \cdot \nabla\left|A_{p}\right\rangle=(1-\mathrm{P}) \boldsymbol{v} \cdot \nabla\left|A_{p}\right\rangle .
$$


One has

$$
\begin{aligned}
\mathrm{P} \boldsymbol{v} \cdot \nabla\left|A_{\boldsymbol{p}}\right\rangle & =\left|A_{\mu}\right\rangle\left\langle A^{\mu}|\boldsymbol{v} \cdot \nabla| A_{\boldsymbol{p}}\right\rangle \\
& =\left|A_{n}\right\rangle\left(\mathrm{i} \boldsymbol{\Omega}_{\boldsymbol{v}}\right)_{p}^{n}+\left|A_{T}\right\rangle\left(\mathrm{i} \boldsymbol{\Omega}_{\boldsymbol{v}}\right)_{p}^{\mathrm{T}} \\
& =\frac{1}{3} T^{-1}\left|v^{2}\right\rangle \nabla .
\end{aligned}
$$

Thus,

and then

$$
\mathrm{Q} v \cdot \nabla\left|A_{p}\right\rangle=T^{-1}\left|v \boldsymbol{v}-\frac{1}{3} v^{2} \boldsymbol{I}\right\rangle \cdot \nabla
$$

$$
\eta_{p}^{p} \cdot \Delta p=\bar{n}^{-1} \nabla \cdot \Delta \pi
$$

where the linearized stress tensor is

$$
\Delta \pi=-\bar{n} m \boldsymbol{m}: \nabla \Delta \boldsymbol{u}
$$

and the fourth-rank tensor $\boldsymbol{m}$ is

$$
\boldsymbol{m} \doteq v_{\mathrm{t}}^{-2}\left\langle\boldsymbol{v} \boldsymbol{v}-\frac{1}{3} v^{2} \boldsymbol{I}\left|\widehat{\mathrm{C}}^{-1}\right| \boldsymbol{v} \boldsymbol{v}-\frac{1}{3} v^{2} \boldsymbol{I}\right\rangle .
$$

This is the same result that follows from the traditional approach described in appendix A. There the matrix element is written in terms of $\boldsymbol{w} \doteq \boldsymbol{v}-\boldsymbol{u}$, where $\boldsymbol{u}$ is the lowest-order flow velocity. Here we are perturbing from an equilibrium with no flow, so $\boldsymbol{w} \rightarrow \boldsymbol{v}$.

The quantity $\left|m \boldsymbol{v} v-(1 / 3) m v^{2} \boldsymbol{I}\right\rangle$ (see (2.66)) is called the subtracted momentum flux; the subtraction arises from the $\mathrm{P}$ in $\mathrm{Q}=1-\mathrm{P}$. All dissipative transport coefficients are defined in terms of subtracted fluxes; the physical reason is that the $\mathrm{P}$ terms represent the fluxes that already exist in local thermal equilibrium, and those must be subtracted from the total flux in order to obtain the gradient-driven corrections that are responsible for the net transport (which determines the relaxation of a small perturbation to thermal equilibrium). In classical Chapman-Enskog theory (appendix A), subtracted fluxes arise when the first-order solvability conditions (Euler equations) are used to simplify the correction equations by eliminating time derivatives in favour of spatial gradients. The use of projection operators executes that task substantially more efficiently.

The reduction of $\boldsymbol{m}$ to a form involving a single scalar viscosity coefficient $\mu$ is given in appendix A. One thus recovers the proper expression $(2.8 a)$ for the linearized stress tensor $\Delta \pi$ of the unmagnetised OCP, where $\pi$ is defined by (2.5). Similar considerations lead to $(2.8 b)$ for the linearized heat flow.

I remind the reader that the present calculation of the dissipative fluxes is correct only to first order in the gradients. Second-order fluxes are discussed in Part 2.

\section{The Braginskii equations: Multispecies, magnetised, classical, collisional fluid equations}

I now turn to the important problem of classical transport in multispecies, magnetised plasma (in the limit of weak coupling). This is somewhat more technically complicated than is the OCP because of interspecies collisional coupling and the loss of symmetry due to the magnetic field, but the basic idea of projection into hydrodynamic and orthogonal subspaces still applies. Again, in this Part 1 I consider only linear response and first-order dissipative effects. Nonlinear equations and Burnett corrections will be considered in Part 2. 
3.1. Exact form of the moment equations, and summary of the results for a two-species plasma

Before I consider perturbations from thermal equilibrium, it is useful to have the exact form of the moment equations in mind. The starting point will be the Landau kinetic equation for the one-particle distribution, including the effect of an external magnetic field $\boldsymbol{B}$ (assumed here to be constant in space):

$$
\partial_{t} f_{s}+\boldsymbol{v} \cdot \nabla f_{s}+\left(\boldsymbol{E}+c^{-1} \boldsymbol{v} \times \boldsymbol{B}\right) \cdot \boldsymbol{\partial}_{s} f=-\mathrm{C}_{s}[f],
$$

where as usual $\boldsymbol{E}_{\boldsymbol{k}}=\sum_{\bar{s}}(n q)_{\bar{s}} \int \mathrm{d} \overline{\boldsymbol{v}} \boldsymbol{\epsilon}_{\boldsymbol{k}} f_{\bar{s}, \boldsymbol{k}}(\overline{\boldsymbol{v}}), \boldsymbol{\partial}_{s} \doteq(q / m)_{s} \partial_{\boldsymbol{v}}$, and C is the Landau collision operator defined in (B 1). That operator conserves number density (separately for each species), total (summed over species) momentum density and total kinetic-energy density:

$$
\sum_{\bar{s}} \bar{n}_{\bar{s}} \int \mathrm{d} \overline{\boldsymbol{v}}\left(\begin{array}{c}
\delta_{s \bar{s}} \\
m_{\bar{s}} \overline{\boldsymbol{v}} \\
\frac{1}{2} m_{\bar{s}} \bar{v}^{2}
\end{array}\right) \mathrm{C}_{\bar{s}}[f]=0 .
$$

Upon taking the number-density, momentum-density and kinetic-energy-density moments of (3.1), one is led to

$$
\begin{gathered}
\partial_{t} n_{s}+\nabla \cdot\left(n_{s} \boldsymbol{u}_{s}\right)=0, \\
(n m)_{s} \frac{\mathrm{d}_{s} \boldsymbol{u}_{s}}{\mathrm{~d} t}=(n q)_{s}\left(\boldsymbol{E}+c^{-1} \boldsymbol{u}_{s} \times \boldsymbol{B}\right)-\nabla p_{s}-\nabla \cdot \boldsymbol{\pi}_{s}+\boldsymbol{R}_{s}, \\
\frac{3}{2} n_{s} \frac{\mathrm{d}_{s} T_{s}}{\mathrm{~d} t}=-p_{s} \boldsymbol{\nabla} \cdot \boldsymbol{u}_{s}-\nabla \cdot \boldsymbol{q}_{s}-\boldsymbol{\pi}_{s}: \boldsymbol{S}_{s}+Q_{s},
\end{gathered}
$$

where

$$
\begin{gathered}
p \doteq n T, \\
\frac{\mathrm{d}_{s}}{\mathrm{~d} t} \doteq \frac{\partial}{\partial t}+\boldsymbol{u}_{s} \cdot \nabla, \\
\boldsymbol{S} \doteq \frac{1}{2}\left[\nabla \boldsymbol{u}+(\boldsymbol{\nabla} \boldsymbol{u})^{\mathrm{T}}\right],
\end{gathered}
$$

and the stress tensor $\boldsymbol{\pi}$, the interspecies momentum transfer $\boldsymbol{R}$, the heat-flow vector $\boldsymbol{q}$ and the heat generation $Q$ are defined by

$$
\begin{aligned}
\boldsymbol{\pi}_{s} & \doteq \int \mathrm{d} \boldsymbol{v}(\bar{n} m \boldsymbol{w})_{s} \boldsymbol{w}_{s} f_{s}-p_{s} \boldsymbol{I}, \\
\boldsymbol{R}_{s} & \doteq-\int \mathrm{d} \boldsymbol{v}(\bar{n} m \boldsymbol{w})_{s} \mathrm{C}_{s}[f] \\
\boldsymbol{q}_{s} & \doteq \int \mathrm{d} \boldsymbol{v}\left(\frac{1}{2} \bar{n} m w^{2}\right)_{s} \boldsymbol{w}_{s} f_{s}, \\
Q_{s} & \doteq-\int \mathrm{d} \boldsymbol{v}\left(\frac{1}{2} \bar{n} m w^{2}\right)_{s} \mathrm{C}_{s}[f],
\end{aligned}
$$

with $\boldsymbol{w}_{s} \doteq \boldsymbol{v}-\boldsymbol{u}_{s}$. Braginskii uses the notation $\boldsymbol{R} \equiv \boldsymbol{R}_{e}$. Conservation of total momentum ensures that $\sum_{i} \boldsymbol{R}_{i}=-\boldsymbol{R}$. For a one-component plasma, $\boldsymbol{R}$ vanishes because the collision operator conserves momentum for like-species collisions; similarly, $Q$ vanishes 
for the OCP by energy conservation. In the general case, application of the conservation laws leads to

$$
\sum_{i} Q_{i}=-Q_{e}-\sum_{i} \boldsymbol{R}_{i} \cdot\left(\boldsymbol{u}_{e}-\boldsymbol{u}_{i}\right)
$$

which reduces to

$$
Q_{e}=-Q_{i}+\boldsymbol{R} \cdot \boldsymbol{u}
$$

$\left(\boldsymbol{u} \doteq \boldsymbol{u}_{e}-\boldsymbol{u}_{i}\right)$ for a single species of ions.

For a two-species, strongly magnetised electron-ion plasma (charges $q_{e}=-e$ and $q_{i}=Z e$ ) possessing overall charge neutrality and with mass ratio $\mu \ll 1$ and $\left(v /\left|\omega_{\mathrm{c}}\right|\right)_{s} \ll 1$, Braginskii quotes the following results, which constitute an approximate hydrodynamic closure (the numerical coefficients are valid for $Z=1$ ). Further discussion of the limits of validity of this closure is given at the end of this section.

(i) The electron and ion collision times are

$$
\tau_{e}=\frac{3 m_{e}^{1 / 2} T_{e}^{3 / 2}}{4 \sqrt{2 \pi} \ln \Lambda Z^{2} e^{4} n_{i}}, \quad \tau_{i}=\frac{3 m_{i}^{1 / 2} T_{i}^{3 / 2}}{4 \sqrt{2 \pi} \ln \Lambda Z^{4} e^{4} n_{i}} .
$$

(ii) The gyrofrequencies are $\omega_{c s} \doteq(q B / m c)_{s}$. (In my convention, unlike Braginskii's, $\omega_{\mathrm{ce}}$ is negative.) The gyroradii are $\rho_{s} \doteq v_{\mathrm{ts}} /\left|\omega_{\mathrm{cs}}\right|$, where $v_{\mathrm{ts}} \doteq(T / m)_{s}^{1 / 2}$.

(iii) The interspecies momentum transfer is denoted by $\boldsymbol{R}_{e} \equiv \boldsymbol{R}$ and $\boldsymbol{R}_{i}=-\boldsymbol{R}$. It consists of two parts, $\boldsymbol{R}=\boldsymbol{R}_{\boldsymbol{u}}+\boldsymbol{R}_{T}$ :

(1) The friction force $\boldsymbol{R}_{\boldsymbol{u}}$ is, with $\boldsymbol{u} \doteq \boldsymbol{u}_{e}-\boldsymbol{u}_{i}$,

$$
\boldsymbol{R}_{\boldsymbol{u}}=-(m n)_{e} \tau_{e}^{-1}\left(\alpha \boldsymbol{u}_{\|}+\boldsymbol{u}_{\perp}\right),
$$

where $\alpha \doteq 0.51$.

(2) The thermal force $\boldsymbol{R}_{T}$ is

$$
\boldsymbol{R}_{T}=-\beta n_{e} \nabla_{\|} T_{e}+\frac{3}{2} \frac{n_{e}}{\omega_{\mathrm{ce}} \tau_{e}} \widehat{\boldsymbol{b}} \times \nabla T_{e},
$$

where $\beta \doteq 0.71$.

(iv) The electron heat flux is $\boldsymbol{q}_{e}=\boldsymbol{q}_{e, \boldsymbol{u}}+\boldsymbol{q}_{e, T}$, where

$$
\begin{gathered}
\boldsymbol{q}_{e, \boldsymbol{u}}=\beta(n T)_{e} \boldsymbol{u}_{\|}-\frac{3}{2} \frac{(n T)_{e}}{\omega_{\mathrm{ce}} \tau_{e}} \widehat{\boldsymbol{b}} \times \boldsymbol{u}, \\
\boldsymbol{q}_{e, T}=-n_{e}\left(\kappa_{\| e} \nabla_{\|} T_{e}+\kappa_{\perp e} \nabla_{\perp} T_{e}+\frac{5}{2} \frac{v_{\mathrm{te}}^{2}}{\omega_{\mathrm{c} e}} \widehat{\boldsymbol{b}} \times \nabla T_{e}\right),
\end{gathered}
$$

where $\kappa_{\| e}=3.16 v_{\mathrm{te}}^{2} \tau_{e}$ and $\kappa_{\perp e}=4.66 \rho_{e}^{2} / \tau_{e}$. (The coefficient 4.66 is derived in appendix D.)

(v) The ion heat flux is

$$
\boldsymbol{q}_{i}=-n_{e}\left(\kappa_{\| i} \nabla_{\|} T_{e}+\kappa_{\perp i} \nabla_{\perp} T_{e}+\frac{5}{2} \frac{v_{\mathrm{ti}}^{2}}{\omega_{\mathrm{c} i}} \widehat{\boldsymbol{b}} \times \nabla T_{e}\right),
$$

where $\kappa_{\| i}=3.9 v_{\mathrm{ti}}^{2} \tau_{i}$ and $\kappa_{\perp i}=2 \rho_{i}^{2} / \tau_{i}$. 
(vi) The ion heat generation is

$$
Q_{i}=Q_{\Delta} \doteq 3\left(\frac{m_{e}}{m_{i}}\right) \tau_{e}^{-1} n_{e}\left(T_{e}-T_{i}\right)
$$

(vii) The electron heat generation is

$$
Q_{e}=-\boldsymbol{R} \cdot \boldsymbol{u}-Q_{\Delta}
$$

(viii) The stress tensor and viscosity coefficients are discussed in appendix E.

In addition to the basic mass-ratio and magnetic-field orderings, Braginskii's equations have a restricted regime of validity. Even for linear response, they are correct only to first order in the gradients. Nonlinearly, as stressed by Mikhaǐlovskir (1967), Mikhǎ̌lovskiǐ \& Tsypin (1971, 1984) and Catto \& Simakov (2004), Braginskii's results correspond to a high-flow regime in which the mean flow velocity $\boldsymbol{u}$ is of the order of the ion sound speed. When $\boldsymbol{u}$ is ordered smaller, additional terms must be retained. (These points are not made clear in Braginskii's article; they are discussed in Part 2.)

\subsection{The hydrodynamic projection for multispecies and magnetised plasma}

The present goal is to rederive the linearized version of Braginskii's closure by using projection methods. However, before proceeding with a hydrodynamic projection, one must decide whether to derive one-fluid or $S$-fluid equations, where $S$ is the number of species. Because the null space of the collision operator is five-dimensional, it would be simplest to project into a five-dimensional, one-fluid hydrodynamic subspace. The resulting single-fluid equations would be identical in form to those derived earlier for the OCP except for the presence of the Lorentz force term. Unfortunately, such a one-fluid hydrodynamics disguises the important fact that when the mass ratio is small the physics of the electrons and the ions are quite different. Thus, following Braginskii, I shall derive a set of $S$-fluid equations. However, this leads to technical complications because one now has $5 S$ equations although the null space remains merely five-dimensional. A consequence is that the interspecies collisional coupling described by $\boldsymbol{R}$ and $Q$ results in some eigenvalues of the linearized problem being of the order of a collision frequency $v$ (implying fast relaxation of some modes on the kinetic time scale) instead of being proportional to $k^{2}$ (implying slow relaxation constrained by conservation laws).

To begin defining the relevant projection operator, let me introduce the natural scalar product, which is the generalization of $(2.13 c)$ to include a species summation:

$$
\langle A|\mathrm{~L}| B\rangle \doteq \sum_{\bar{s}} \int \mathrm{d} \overline{\boldsymbol{v}} \sum_{\bar{s}^{\prime}} \int \mathrm{d} \overline{\boldsymbol{v}}^{\prime} A_{\bar{s}}(\overline{\boldsymbol{v}}) L_{\overline{s s^{\prime}}}\left(\overline{\boldsymbol{v}}, \overline{\boldsymbol{v}}^{\prime}\right) B_{\bar{s}}(\overline{\boldsymbol{v}}) f_{\mathrm{M}, \bar{s}^{\prime}}\left(\overline{\boldsymbol{v}}^{\prime}\right) .
$$

This is natural for several reasons:

(i) The linearized collision operator (times $\bar{n}$ ) is self-adjoint with respect to it:

$$
\langle\psi|\bar{n} \widehat{\mathrm{C}}| \chi\rangle=\langle\chi|\bar{n} \widehat{\mathrm{C}}| \psi\rangle
$$

(see appendix B). 
(ii) For $\boldsymbol{A}$ defined as in (2.25), the conservation properties of $\widehat{\mathrm{C}}$ take the simple form $^{22}$

$$
\langle\bar{n} \boldsymbol{A}| \widehat{\mathrm{C}}=0 .
$$

(iii) The first-order electric field follows as

$$
\Delta \boldsymbol{E}_{k}=\left\langle\mathbb{E}_{k} \mid \chi_{k}\right\rangle,
$$

where

$$
\mathbb{E}_{k, s \bar{s}}(\boldsymbol{v}, \overline{\boldsymbol{v}}) \doteq(\bar{n} q)_{\bar{s}} \boldsymbol{\epsilon}_{\boldsymbol{k}}
$$

(independent of $s, \boldsymbol{v}$, and $\overline{\boldsymbol{v}}$ ).

Although projections are often discussed in terms of scalar products, and the scalar product (3.15) is natural from several points of view, its use in defining an $S$-species projection operation is somewhat problematical because of the implied species summation. If one insists on defining bras and kets in terms of a scalar product, that summation must sometimes be inhibited in one way or another in order that one end up with a species-dependent result. A definition that generalizes naturally to the $\Gamma$-space theory discussed in Part 2 is the following. Subsume the species index into a generalized field index: $\boldsymbol{A}_{s} \equiv A_{s}^{\mu} \rightarrow A^{\mu}$. (When a species index is written explicitly, then the superscript refers to just the usual field index.) Then define

$$
\mathrm{P} \doteq\left|A^{\mu}\right\rangle M_{\mu \mu^{\prime}}^{-1}\left\langle A^{\mu^{\prime}}\right|
$$

Also define

$$
\Delta a^{\mu}=\left\langle A^{\mu} \mid \Delta \chi\right\rangle .
$$

Here one is treating $\Delta \chi$ as the collection of all $\Delta \chi_{s}$ values. Normally, the natural scalar product would require that the $s$ be changed to $\bar{s}$ and a summation over $\bar{s}$ be done. However, $A^{\mu}$ depends on a specific species $s_{\mu}$, not the entire collection $A_{s}^{\mu}$ for all $s$. A consistent interpretation is that the presence of a specific species index inhibits the species summation in the scalar product. Effectively, $A_{s_{\mu}}^{\mu}$ behaves inside a scalar product as the $s$-dependent quantity $\delta_{s_{\mu}(s)} A_{(s)}^{\mu}$, where parentheses inhibit the summation convention. Then

$$
\left\langle A^{\mu} \mid \Delta \chi\right\rangle=\sum_{\bar{s}} \int \mathrm{d} \overline{\boldsymbol{v}} \delta_{s_{\mu} \bar{s}} A_{\bar{s}}^{\mu}(\overline{\boldsymbol{v}}) \Delta \chi_{\bar{s}}(\overline{\boldsymbol{v}})=\int \mathrm{d} \overline{\boldsymbol{v}} A_{s_{\mu}}^{\mu}(\overline{\boldsymbol{v}}) \Delta \chi_{s_{\mu}}(\overline{\boldsymbol{v}}) \doteq \Delta a^{\mu} .
$$

To extract all hydrodynamic field components of specific species $s$, I shall use the notation

$$
\boldsymbol{a}_{s}=\left\langle\boldsymbol{A} 1_{s} \mid \Delta \chi\right\rangle,
$$

where the $1_{s}$ inhibits the species summation in the scalar product.

Because $\boldsymbol{A}$ is defined in terms of purely kinetic quantities that do not couple species, in fact $M_{s s^{\prime}}^{\mu \mu^{\prime}}$ is diagonal in the species indices. It is also diagonal in the field indices

${ }^{22}$ The species sum included in the definition (3.15) is crucial to this result. 
because of the orthogonality built into the definition of $\boldsymbol{A}$ (see (2.28)). Thus, $M^{\mu \mu^{\prime}}=$ $M^{(\mu)} \delta_{(\mu) \mu^{\prime}}\left(M^{\mu} \doteq M^{(\mu)(\mu)}\right)$ and

$$
\mathrm{P}=\sum_{\mu}\left|A^{\mu}\right\rangle M_{\mu}^{-1}\left\langle A^{\mu}\right|
$$

In Part 2, potential-energy contributions will be added to $\boldsymbol{A}, \boldsymbol{M}$ will no longer be diagonal, and (a space-dependent generalization of) (3.20) will be used.

Define the magnetic-field operator by

$$
\mathrm{i} \widehat{\mathbf{M}} \doteq c^{-1} \boldsymbol{v} \times \boldsymbol{B} \cdot \boldsymbol{\partial}=\omega_{\mathrm{c}} \frac{\partial}{\partial \zeta},
$$

where $\zeta$ is the negative ${ }^{23}$ of the polar angle in velocity space. It is then a straightforward exercise to show that the kinetic equation linearized around an absolute Maxwellian can be written as

$$
\partial_{t}|\Delta \chi\rangle+\mathrm{i} \mathcal{L}|\Delta \chi\rangle=0
$$

where

$$
\mathcal{L} \doteq \mathrm{L}+\mathrm{L}_{E}+\mathrm{L}_{\mathrm{M}}, \quad \mathrm{L} \doteq \mathrm{L}_{v}+\mathrm{L}_{\mathrm{C}}
$$

with $\mathrm{L}_{\mathrm{M}} \doteq \widehat{\mathrm{M}}$. The projections of the kinetic equation are formally the same as (2.43) and (2.45). Working out the components of the frequency matrix is straightforward. There are no surprises for $\mathrm{i} \boldsymbol{\Omega}_{\boldsymbol{v}}$; it leads to the same (species-dependent) Euler contributions as for the OCP. It is easy to show that $\left(\mathrm{i} \boldsymbol{\Omega}_{\mathrm{M}}\right)_{p}^{p}$ gives rise to the usual Lorentz force, while all other magnetic contributions to the frequency matrix vanish. ${ }^{24}$ It is shown in appendix B that

$$
\mathrm{i} \boldsymbol{\Omega}_{\mathrm{C}} \cdot \Delta \boldsymbol{a}=m_{s} \sum_{s^{\prime}} v_{s s^{\prime}}\left(\Delta \boldsymbol{u}_{s}-\Delta \boldsymbol{u}_{s^{\prime}}\right)+3 \sum_{s^{\prime}}\left(\frac{m_{s}}{M_{s s^{\prime}}}\right) v_{s s^{\prime}}\left(\Delta T_{s}-\Delta T_{s^{\prime}}\right),
$$

where $v_{s s^{\prime}}$ is a generalized interspecies collision frequency defined by (B 30) and $M_{s s^{\prime}} \doteq m_{s}+m_{s^{\prime}}$. At this point, we have obtained

$$
\begin{gathered}
\frac{\partial}{\partial t}\left(\frac{\Delta n}{\bar{n}}\right)_{s}=-\nabla \cdot \Delta \boldsymbol{u}_{s} \\
(\bar{n} m)_{s} \frac{\partial \Delta \boldsymbol{u}_{s}}{\partial t}=(\bar{n} q)_{s}\left(\Delta \boldsymbol{E}+c^{-1} \Delta \boldsymbol{u}_{s} \times \boldsymbol{B}\right)-\nabla \Delta p_{s}-(\bar{n} m)_{s} \sum_{s^{\prime}} v_{s s^{\prime}}\left(\Delta \boldsymbol{u}_{s}-\Delta \boldsymbol{u}_{s^{\prime}}\right) \\
-\left\langle\bar{n} \boldsymbol{P}^{\prime} 1_{s}|\mathrm{PiLQ}| \mathrm{Q} \Delta \chi\right\rangle,
\end{gathered}
$$

\footnotetext{
${ }^{23} \zeta$ is defined such that it increases with time during ion gyration. A diagram illustrating the coordinates of a gyrospiralling particle, originally published as figure 1 of Krommes (2012), can be found on Sheldon's whiteboard in Episode 14, Season 6 (January 31, 2013) of CBS's The Big Bang Theory.

${ }^{24} \widehat{M}$ conserves number and kinetic energy, but not vector momentum. However, the momentum integral of $\widehat{M}$ lives solely in the hydrodynamic subspace. That is, upon integrating by parts,
}

$$
\left\langle A_{s}^{\boldsymbol{p}}\left|\widehat{\mathrm{M}}_{s}\right| \Delta \chi_{s}\right\rangle=\left\langle m_{s} \boldsymbol{v}\left|\omega_{\mathrm{cs}} \boldsymbol{v} \times \widehat{\boldsymbol{b}} \cdot \partial_{\boldsymbol{v}}\right| \Delta \chi_{s}\right\rangle=-\left(m \omega_{\mathrm{c}}\right)_{s}\left\langle\boldsymbol{v} \times \widehat{\boldsymbol{b}} \mid \Delta \chi_{s}\right\rangle=-\omega_{\mathrm{cs}} \Delta \boldsymbol{p}_{s} \times \widehat{\boldsymbol{b}} .
$$




$$
\begin{aligned}
\frac{3}{2} \bar{n}_{s} \frac{\partial \Delta T_{s}}{\partial t}= & -p_{s} \nabla \cdot \Delta \boldsymbol{u}_{s}-3 \bar{n}_{s} \sum_{s^{\prime}}\left(\frac{m_{s}}{M_{s s^{\prime}}}\right) v_{s s^{\prime}}\left(\Delta T_{s}-\Delta T_{s^{\prime}}\right) \\
& -\left\langle\bar{n} K^{\prime} 1_{s}|\operatorname{PiLQ}| \mathrm{Q} \Delta \chi\right\rangle .
\end{aligned}
$$

Obviously, the forms of the linearized fluid equations are beginning to emerge, with the parts involving $|\mathrm{Q} \Delta \chi\rangle$ to be determined by closure. Note that only $\mathrm{L} \doteq \mathrm{L}_{v}+\mathrm{L}_{\mathrm{C}}$ enters those parts; it is easy to show that the magnetic-field operator does not couple the subspaces, so $\mathrm{Pi} \widehat{\mathrm{M}} \mathrm{Q}=\mathrm{Qi} \widehat{\mathrm{MP}}=0$, and a similar result holds for $\mathrm{L}_{E}$. Also note that the frequency-matrix term involving $\Delta \boldsymbol{u}_{s}-\Delta \boldsymbol{u}_{s^{\prime}}$ in (3.29b) is not, in general, the complete contribution to the interspecies momentum transfer. As is well known (Braginskii 1965), the effective collision frequency for the parallel momentum transfer differs by a numerical factor from the $v_{s s^{\prime}}$ defined by (B 30); for $Z=1$, that factor is $\alpha=0.51$. The physics is that perturbations to an absolute Maxwellian background do not in general merely produce a shifted Maxwellian; the $v^{-3}$ dependence of the electron-ion collision rate leads to a high-energy tail that enhances the parallel current for fixed electric field. This manifests as a reduction in the effective $v_{s s^{\prime}, \|}$. This effect is not seen in the frequency-matrix portion of the hydrodynamic projection, which only involves the perturbations of the quantities $n, \boldsymbol{u}$ and $T$ that would appear in a local Maxwellian distribution. Thus, the physics of the high-energy tail must be contained in the last, $|\mathrm{Q} \Delta \chi\rangle$ term of (3.29b), as I shall now demonstrate.

\subsection{Hydrodynamic closure}

The straightforward generalization of (2.54) to the multispecies, magnetised case is

$$
\eta_{\nu}^{\mu} \doteq \int_{0}^{\infty} \mathrm{d} \tau \Sigma_{\nu}^{\mu}(\tau) \approx\left\langle A^{\mu}\left|\mathrm{LQ}[\mathrm{Q}(\mathrm{i} \widehat{\mathrm{M}}+\widehat{\mathrm{C}}) \mathrm{Q}]^{-1} \mathrm{QL}\right| A_{\nu}\right\rangle,
$$

the only difference being the addition of the magnetic-field operator to $\widehat{\mathrm{C}}{ }^{25} \mathrm{~A}$ different way of representing the content of (3.30) is to rewrite the solution for the orthogonal projection,

$$
|\mathrm{Q} \Delta \chi\rangle \approx-[\mathrm{Q}(\mathrm{i} \widehat{\mathrm{M}}+\widehat{\mathrm{C}}) \mathrm{Q}]^{-1} \mathrm{QiLP}|\mathrm{P} \Delta \chi\rangle
$$

as the equation

$$
(\mathrm{i} \widehat{\mathrm{M}}+\mathrm{QC})|\mathrm{Q} \Delta \chi\rangle=-\mathrm{QiLP}|\mathrm{P} \Delta \chi\rangle .
$$

Here the result $\mathrm{Qi} \widehat{M Q}=(1-\mathrm{P}) \mathrm{i} \widehat{\mathrm{M}} \mathrm{Q}=\mathrm{i} \widehat{\mathrm{M}} \mathrm{Q}$ was used. Unlike in the OCP, one cannot reduce $\mathrm{QCQ} \rightarrow \widehat{\mathrm{C}}$ because the present hydrodynamic projection inhibits the species summation, so $\mathrm{PC} \neq 0$. Following Braginskii, I now restrict the calculation to a single species of ions. Then one finds

$$
\begin{aligned}
\mathrm{QiLP}|\mathrm{P} \Delta \chi\rangle= & \frac{1}{2}\left|\frac{\boldsymbol{v} \boldsymbol{v}-\left(v^{2} / 3\right) \boldsymbol{I}}{v_{\mathrm{t}}^{2}}\right\rangle: \boldsymbol{W}[\Delta \boldsymbol{u}]+\left|\left(\frac{1}{2} \frac{v^{2}}{v_{\mathrm{t}}^{2}}-\frac{5}{2}\right) \boldsymbol{v}\right\rangle \cdot \nabla\left(\frac{\Delta T}{T}\right) \\
& +\mathrm{Q} \widehat{\mathrm{C}}|\mathrm{P} \Delta \chi\rangle,
\end{aligned}
$$

\footnotetext{
${ }^{25}$ In standard unmagnetized Chapman-Enskog theory (appendix A), the collision operator is ordered large, whereas in practice it is often the case that $i \widehat{M} \gg \widehat{C}\left(\omega_{\mathrm{c}} \gg v\right)$. The operators $i \widehat{M}$ and $\widehat{\mathrm{C}}$ appear symmetrically in (3.30) because no assumption is made about their relative size. One might call this a maximal ordering. However, the best way of thinking about the present methodology is not to focus on orderings of $i \widehat{\mathrm{M}}$ or $\widehat{\mathrm{C}}$, but rather to recognize that one is taking the hydrodynamic limit $\omega, k \rightarrow 0$. The operators $\mathrm{i} \widehat{\mathrm{M}}$ and $\widehat{\mathrm{C}}$ survive that limit because they do not go to zero with $k$.
} 
where the traceless tensor used by Braginskii is

$$
\boldsymbol{W}[\boldsymbol{u}] \doteq \nabla \boldsymbol{u}+(\boldsymbol{\nabla u})^{\mathrm{T}}-\frac{2}{3}(\nabla \cdot u) \boldsymbol{l} .
$$

To simplify the last term of (3.33), use $\mathrm{Q} \widehat{\mathrm{C} P}=\widehat{\mathrm{C} P}-\mathrm{P} \widehat{\mathrm{CP}}$, where the last term is evaluated in $\S \mathrm{B} .2$ and is given by (B 29). The quantity $\widehat{\mathrm{CP}}|\Delta \chi\rangle$ is evaluated in $\S \mathrm{B} .3$ for small mass ratio. For the electrons, the $\Delta T$ part of QC仓P $|\Delta \chi\rangle$ vanishes to lowest order in the mass ratio; for the ions, $\mathrm{Q} \widehat{\mathrm{CP}}|\Delta \chi\rangle$ vanishes altogether to lowest order. Thus, with the results of appendix B, all pieces of (3.32) for $\mathrm{Q}|\Delta \chi\rangle$ are known. To rearrange that equation into Braginskii's form, use $\mathrm{Q} \widehat{\mathrm{C}}=\widehat{\mathrm{C}}-\mathrm{PC}$ and place the $\mathrm{PC}$ terms on the right-hand side. For the electrons, one finds (cf. Braginskii's equation (4.12))

$$
\begin{aligned}
-\left(\mathrm{i} \widehat{\mathrm{M}}+\widehat{\mathrm{C}}_{e}\right)|\mathrm{Q} \Delta \chi\rangle= & \frac{1}{2}\left|\frac{\boldsymbol{v} \boldsymbol{v}-\left(v^{2} / 3\right) \boldsymbol{I}}{v_{\mathrm{te}}^{2}}\right\rangle: \boldsymbol{W}\left[\Delta \boldsymbol{u}_{e}\right]+\left|\left(\frac{1}{2} \frac{v^{2}}{v_{\mathrm{te}}^{2}}-\frac{5}{2}\right) \boldsymbol{v}\right\rangle \cdot \nabla\left(\frac{\Delta T}{T}\right)_{e} \\
& +\frac{1}{v_{\mathrm{te}}^{2} \tau_{e}}\left|\left[3 \sqrt{\frac{\pi}{2}}\left(\frac{v_{\mathrm{te}}}{v}\right)^{3}-1\right] \boldsymbol{v}\right\rangle \cdot \Delta \boldsymbol{u}-|\boldsymbol{v}\rangle \frac{1}{v_{\mathrm{te}}^{2}}\left\langle 1_{e} \boldsymbol{v}\left|\widehat{\mathrm{C}}^{\mathrm{Lor}}\right| \mathrm{Q} \Delta \chi\right\rangle,
\end{aligned}
$$

where the last term is the Lorentz approximation to PCQ $|\Delta \chi\rangle$; only the momentum projection appears because the Lorentz collision operator conserves kinetic energy. The coefficient of that term is just the lowest-order approximation to the electron momentum transfer:

$$
-|\boldsymbol{v}\rangle \frac{1}{v_{\mathrm{te}}^{2}}\left\langle 1_{e} \boldsymbol{v}\left|\widehat{\mathrm{C}}^{\mathrm{Lor}}\right| \mathrm{Q} \Delta \chi\right\rangle=\frac{1}{\bar{n} T}|\boldsymbol{v}\rangle \cdot \Delta \boldsymbol{R},
$$

where $($ see $(3.5 b))$

$$
\Delta \boldsymbol{R} \doteq-\left\langle(\bar{n} m)_{e} \boldsymbol{v} 1_{e}\left|\widehat{\mathrm{C}}^{\mathrm{Lor}}\right| \mathrm{Q} \Delta \chi\right\rangle .
$$

For the ions, one finds that the Q $\widehat{\mathrm{CP}}$ term in (3.33) is negligible for small mass ratio, so (cf. Braginskii's equation (4.15))

$$
-\left(\mathrm{i} \widehat{\mathrm{M}}+\widehat{\mathrm{C}}_{i i}\right)|\mathrm{Q} \Delta \chi\rangle=\frac{1}{2}\left|\frac{\boldsymbol{v} \boldsymbol{v}-\left(v^{2} / 3\right) \boldsymbol{I}}{v_{\mathrm{ti}}^{2}}\right\rangle: \boldsymbol{W}\left[\Delta \boldsymbol{u}_{i}\right]+\left|\left(\frac{1}{2} \frac{v^{2}}{v_{\mathrm{ti}}^{2}}-\frac{5}{2}\right) \boldsymbol{v}\right\rangle \cdot \nabla\left(\frac{\Delta T}{T}\right)_{i} ;
$$

this is identical in form to the correction equation for the OCP (see (A 18b)).

Thus, the projection-operator methodology restricted to first-order processes has reproduced Braginskii's correction equations - as, of course, it must since physics content is invariant to mathematical representation. Although we have not obtained any new results, it is hoped that the use of projection operators clarifies the underlying structure of the transport equations, the key import of the null eigenspace, and the distinction between a perturbed local Maxwellian distribution and the true perturbed distribution that includes a high-energy, non-Maxwellian tail driven by the various thermodynamic forces.

From this point forward, the route to the final values of the transport coefficients, namely the evaluation of the matrix elements (3.30), follows that of Braginskii and 
other authors. In general, numerical or approximate analytical work is required; there is no need to repeat such analysis here. But as an illustration of the content of the correction equations and with the goal of providing further insight into the various orthogonal projections, I show in appendix D how to work out the perpendicular electron heat flow $\boldsymbol{q}_{\perp, e}$ in the limit of small $v_{e} /\left|\omega_{c e}\right|$.

\subsection{Onsager symmetries}

Onsager's symmetry theorem (Onsager 1931a,b; Casimir 1945; Krommes \& Hu 1993) is one of the deepest results in classical statistical physics. It is a statement about the relaxation of an arbitrarily coupled $N$-body system slightly perturbed from a Gibbsian thermal equilibrium. For the transport matrix $\eta$, it reads in the present covariant notation

$$
\widehat{\eta}^{\mu \nu}(\boldsymbol{B})=\widehat{\eta}^{\nu \mu}(-\boldsymbol{B}) .
$$

Here $\hat{\boldsymbol{\eta}} \doteq \boldsymbol{E} \cdot \boldsymbol{\eta}$, where $\boldsymbol{E}$ is the parity matrix such that under a time-reversal transformation $\boldsymbol{A} \rightarrow \boldsymbol{E} \cdot \boldsymbol{A}$. (In a diagonal representation, $E_{(i)}^{(i)}= \pm 1$ depending on whether the $i$ th variable is even or odd under time reversal. For my choice of $\boldsymbol{A}$, $\boldsymbol{E}=\operatorname{diag}[1,-1,1]$ for each species.) Fundamentally, this symmetry is a consequence of the time reversibility of the microscopic dynamics. It is critical to observe that the theorem applies to the fully contravariant (or fully covariant) transport tensor, not the mixed tensor $\boldsymbol{\eta}_{v}^{\mu}$ that appears naturally in the hydrodynamic equations. Failure to recognize this fact has led to confusion in the literature; a thorough discussion is given by Krommes \& Hu (1993, § III).

As a consistency check, I shall sketch a proof that the present representation, involving weakly coupled dynamics represented by the Landau collision operator, possesses Onsager symmetry (as already discussed by Braginskii from a more traditional point of view). The fully contravariant version of (3.30) is

$$
\eta^{\mu \nu}=\left\langle A^{\mu}\left|\mathrm{LQ}[\mathrm{Q}(\mathrm{i} \widehat{\mathrm{M}}+\widehat{\mathrm{C}}) \mathrm{Q}]^{-1} \mathrm{QL}\right| A^{\nu}\right\rangle .
$$

First suppose that instead of $A^{\mu}$ and $A^{\nu}$ one had generic functions $\psi_{s}$ and $\chi_{s}$, where as usual the $s$ is to be summed over in the standard scalar product. (Such functions would arise in a one-fluid hydrodynamics.) Then one could proceed by rearranging the scalar product with the aid of adjoint operators as follows:

$$
F[\psi, \chi ; \boldsymbol{B}] \doteq\left\langle\psi\left|\mathrm{LQ}[\mathrm{Q}(\mathrm{i} \widehat{\mathrm{M}}+\widehat{\mathrm{C}}) \mathrm{Q}]^{-1} \mathrm{QL}\right| \chi\right\rangle=\left\langle\chi\left|\mathrm{L}^{\dagger} \mathrm{Q}^{\dagger}[\mathrm{Q}(\mathrm{i} \widehat{\mathrm{M}}+\widehat{\mathrm{C}}) \mathrm{Q}]^{\dagger-1} \mathrm{Q}^{\dagger} \mathrm{L}^{\dagger}\right| \psi\right\rangle .
$$

With respect to the standard scalar product (which does not include complex conjugation), the operators $\mathrm{Q}, \bar{n} \widehat{\mathrm{C}}, \mathrm{L}_{v} \doteq-\mathrm{i} \boldsymbol{v} \cdot \nabla$ and $\bar{n} \mathrm{~L}_{\mathrm{C}} \doteq-\mathrm{i} \overline{\mathrm{C}} \widehat{\mathrm{C}}$ are self-adjoint, whereas $\widehat{\mathrm{M}}$ is anti-self-adjoint. ${ }^{26}$ Therefore,

$$
F[\psi, \chi ; \boldsymbol{B}]=\left\langle\chi\left|\mathrm{LQ}[\mathrm{Q}(-\mathrm{i} \widehat{\mathrm{M}}+\widehat{\mathrm{C}}) \mathrm{Q}]^{-1} \mathrm{QL}\right| \psi\right\rangle=F[\chi, \psi ;-\boldsymbol{B}] .
$$

This is a restricted form of Onsager's symmetry. However, notice that if $\psi$ and $\chi$ were the hydrodynamic vector $\boldsymbol{A}$, then the properties $\langle\bar{n} \boldsymbol{A}| \widehat{\mathrm{C}}=\mathbf{0}$ (conservation) and $\widehat{\mathrm{C}}|\boldsymbol{A}\rangle=\mathbf{0}$ (null eigenvectors) remove the $\widehat{\mathrm{C}}$ from $\mathrm{L}$ and lead to the representation

$$
F\left[\boldsymbol{A}, \boldsymbol{A}^{\mathrm{T}} ; \boldsymbol{B}\right]=\nabla_{i}\left\langle\boldsymbol{A}\left|v_{i} \mathrm{Q}[\mathrm{Q}(\mathrm{i} \widehat{\mathrm{M}}+\widehat{\mathrm{C}}) \mathrm{Q}]^{-1} \mathrm{Q} v_{j}\right| \boldsymbol{A}^{\mathrm{T}}\right\rangle \nabla_{j} .
$$

\footnotetext{
${ }^{26} \mathrm{~L}_{v}$ and $\widehat{\mathrm{M}}$ are diagonal in the species index, so $\bar{n} \mathrm{~L}_{v}$ and $\bar{n} \widehat{\mathrm{M}}$ possess the same adjoint properties as do $L_{v}$ and $\widehat{M}$.
} 
Given the way $\boldsymbol{A}$ was constructed (its components are orthogonal), symmetry in velocity space implies that there is no cross-coupling between elements with opposite parity. Thus, Onsager's symmetry (3.39) applies for the unhatted form of the transport matrix in this case.

In the more interesting case in which one projects onto a particular species, the transport matrix is constructed from the specific $A^{\mu}$ and $A^{\nu}$, whose species indices $s_{\mu}$ and $s_{v}$ are not to be summed. Because the outer summations are inhibited, one cannot use the self-adjoint property of the $\widehat{\mathrm{C}}$ that appears in the $\mathrm{L}$ operators, nor can one remove $\widehat{\mathrm{C}}$ from L by means of the species-summed conservation property; this implies the existence of non-trivial cross-terms in the transport matrix. I shall illustrate for the important special case of two species. One has

$$
\begin{aligned}
& \eta^{\boldsymbol{p}_{e} T_{e}}=-\left\langle\boldsymbol{P}_{e}^{\prime}\left|\widehat{\mathrm{C}}_{e i} \mathrm{Q}\left(\widehat{\mathrm{D}}^{-1}\right)_{i e} \mathrm{Q}\right| K_{e}^{\prime} \boldsymbol{v}\right\rangle \cdot \nabla, \\
& \eta_{e}^{T_{e} \boldsymbol{p}_{e}}=-\nabla \cdot\left\langle K_{e}^{\prime} \boldsymbol{v}\left|\mathrm{Q}\left(\widehat{\mathrm{D}}^{-1}\right)_{e i} \mathrm{Q} \widehat{\mathrm{C}}_{i e}\right| \boldsymbol{P}_{e}^{\prime}\right\rangle,
\end{aligned}
$$

where $\widehat{\mathrm{D}} \doteq[\mathrm{Q}(-\mathrm{i} \widehat{\mathrm{M}}+\widehat{\mathrm{C}}) \mathrm{Q}]^{-1}$. The inverse of the matrix

$$
\boldsymbol{M} \doteq\left(\begin{array}{ll}
\widehat{\mathrm{A}} & \widehat{\mathrm{B}} \\
\widehat{\mathrm{C}} & \widehat{\mathrm{D}}
\end{array}\right)
$$

where $\widehat{\mathrm{A}}, \widehat{\mathrm{B}}, \widehat{\mathrm{C}}$ and $\widehat{\mathrm{D}}$ are non-commuting operators, is ${ }^{27}$

$$
\boldsymbol{M}^{-1}=\left(\begin{array}{cc}
\left(\widehat{\mathrm{A}}-\widehat{\mathrm{B}} \widehat{\mathrm{D}}^{-1} \widehat{\mathrm{C}}\right)^{-1} & -\left(\widehat{\mathrm{D}} \widehat{\mathrm{B}}^{-1} \widehat{\mathrm{A}}-\widehat{\mathrm{C}}\right)^{-1} \\
-\left(\widehat{\mathrm{A}} \widehat{\mathrm{C}}^{-1} \widehat{\mathrm{D}}-\widehat{\mathrm{B}}\right)^{-1} & \left(\widehat{\mathrm{D}}-\widehat{\mathrm{C}} \widehat{\mathrm{A}}^{-1} \widehat{\mathrm{B}}\right)^{-1}
\end{array}\right)
$$

Because of the complicated form of (3.46), it is not yet obvious that (3.44a) and $(3.44 b)$ are equal to within a sign. To demonstrate that, use the momentum conservation property

$$
\left\langle\bar{n}_{e} \boldsymbol{P}_{e}^{\prime}\right| \widehat{\mathrm{C}}_{e i}+\left\langle\bar{n}_{i} \boldsymbol{P}_{i}^{\prime}\right| \widehat{\mathrm{C}}_{i e}=0
$$

and the result $\eta^{\mathrm{T}_{e} \boldsymbol{P}_{e}}+\eta^{T_{e} \boldsymbol{P}_{i}}=0$, which follows from $\widehat{\mathrm{C}}\left|\boldsymbol{P}^{\prime}\right\rangle=0$, to find

$$
\begin{gathered}
\eta^{\boldsymbol{p}_{e} T_{e}} / T=\bar{n}_{e}^{-1}\left\langle\bar{n}_{i} \boldsymbol{P}_{i}^{\prime}\left|\widehat{\mathrm{C}}_{i e} \mathrm{Q}\left(\widehat{\mathrm{D}}^{-1}\right)_{e e}\right| \gamma_{e} \boldsymbol{v}\right\rangle \cdot \nabla, \\
\eta^{T_{e} \boldsymbol{p}_{e}} / T=\nabla \cdot\left\langle\gamma_{e} \boldsymbol{v}\left|\left(\widehat{\mathrm{D}}^{-1}\right)_{e e} \mathrm{Q} \widehat{\mathrm{C}}_{e i}\right| \boldsymbol{P}_{i}^{\prime}\right\rangle,
\end{gathered}
$$

where $\gamma(\boldsymbol{v}) \doteq(1 / 2) m v^{2} / T-5 / 2$ arises from the calculation of $\mathrm{Q} K^{\prime} \boldsymbol{v}$. Since both expressions now involve the common matrix element ${ }^{28}\left(\widehat{\mathrm{D}}^{-1}\right)_{e e}=\left[\widehat{\mathrm{C}}_{e e}^{\prime}-\right.$ $\left.\widehat{\mathrm{C}}_{e i}^{\prime}\left(\widehat{\mathrm{C}}_{i i}^{\prime}\right)^{-1} \widehat{\mathrm{C}}_{i e}^{\prime}\right]^{-1}$, where $\widehat{\mathrm{C}}^{\prime} \doteq \mathrm{i} \widehat{\mathrm{M}}+\widehat{\mathrm{C}}$ (cf. (3.46)), they can be easily compared. Upon referring to the form (B6) of the linearized Landau operator, one sees that an integration by parts of $\left\langle\bar{n}_{i} \boldsymbol{P}_{i}^{\prime}\right| \widehat{\mathrm{C}}_{i e}$ in the expression (3.48a) introduces a minus sign

${ }^{27}$ When the operators commute, (3.46) correctly reduces to the familiar result

$$
\left(\begin{array}{ll}
A & B \\
C & D
\end{array}\right)^{-1}=\frac{1}{\Delta}\left(\begin{array}{cc}
D & -B \\
-C & A
\end{array}\right)
$$

where $\Delta \doteq A D-B C$.

${ }^{28}$ For small mass ratio, the second term of this element is $O\left(\left(m_{e} / m_{i}\right)^{1 / 2}\right) \ll 1$. 
and that the $(3.48 a, b)$ are otherwise equal with $\boldsymbol{B} \rightarrow-\boldsymbol{B}$. Thus, we have recovered (3.39). It is interesting to contemplate that the microscopic time reversibility used in Onsager's original (and more general) derivation shows up in the above proof as the constraint of macroscopic momentum conservation.

Braginskii remarked upon the Onsager symmetry between the electron temperaturegradient contribution to the friction force and the flow-driven contribution to the electron heat flux. (Those effects are absent for the ions to lowest order in the mass ratio.) He failed to mention that the stress tensor $\pi$ also affords an example of the symmetry. As discussed for the case of the OCP, $\pi$ can be written for infinitesimal perturbations as a fourth-order tensor $\boldsymbol{m}$ applied to $\nabla \Delta \boldsymbol{u}$. The ultimate effect in the momentum equation is $-\nabla \cdot \pi=-\nabla \cdot \boldsymbol{m}:(\nabla \Delta \boldsymbol{u})$, which in Fourier space can be written as $(\boldsymbol{k} \cdot \boldsymbol{m} \cdot \boldsymbol{k}) \cdot \Delta \boldsymbol{u} \rightarrow \eta_{j}^{i} \Delta u^{j}=\eta^{i j} \Delta u_{j}$; here the lowering of the index just involves an index-independent normalization factor. As discussed in appendix E, $\boldsymbol{m}$ is constructed from symmetrized tensor products of the matrices $\boldsymbol{B} \doteq \widehat{\boldsymbol{b}} \widehat{\boldsymbol{b}}, \boldsymbol{\delta}^{\perp} \doteq \boldsymbol{I}-\widehat{\boldsymbol{b}} \widehat{\boldsymbol{b}}$, and $\boldsymbol{\beta} \doteq \widehat{\boldsymbol{b}} \times$. The contributions to $\boldsymbol{\eta}$ that do not involve $\boldsymbol{\beta}$ are easily seen to be symmetric and invariant under a change of sign of $\boldsymbol{B}$. The remaining terms (i.e. the gyroviscous stresses), involve either $\left\{\boldsymbol{\delta}^{\perp} \boldsymbol{\beta}\right\}$ or $\{\boldsymbol{B} \boldsymbol{\beta}\}$, where the symmetrization is denoted by the braces. Thus, the gyroviscous contributions to $\boldsymbol{\eta}$ involve $\boldsymbol{k} \cdot\left\{\boldsymbol{\delta}^{\perp} \boldsymbol{\beta}\right\} \cdot \boldsymbol{k}$ or $\boldsymbol{k} \cdot\{\boldsymbol{B} \boldsymbol{\beta}\} \cdot \boldsymbol{k}$. These tensors are antisymmetric because of the factor of $\boldsymbol{\beta}$, but since the gyroviscous terms are proportional to one power of the signed gyrofrequency, symmetry is restored under the replacement $\boldsymbol{B} \rightarrow-\boldsymbol{B}$. Therefore, all contributions to $\eta^{i j}$ obey the Onsager symmetry (3.39).

\section{Generalized Langevin equation for the hydrodynamics of magnetised plasmas}

The hydrodynamic equations developed in the previous sections describe the ensemble-averaged behaviour of the macroscopic observables. Underlying those equations is a system of generalized Langevin equations for fluctuating quantities whose means are the hydrodynamic variables. Langevin descriptions (Langevin 1908) are fundamental to the theory of both classical and turbulent transport, as they neatly capture and generalize the basic intuition associated with Brownian motion (Wang \& Uhlenbeck 1945), namely, the competition between random excitation and coherent damping. In this section I shall develop a generalized Langevin equation for classical transport. ${ }^{29}$ It will be seen that the transport coefficients are the time integrals of the two-time correlations of the fluctuating forces that appear on the right-hand side of the Langevin equation. Such a representation is fundamental and a core topic in transport theory.

In the previous sections I used the Schrödinger representation, in which the state vector $|\Delta \chi(t)\rangle$ changes with time while the hydrodynamic observables $\langle\boldsymbol{A}|$ are time independent. In this section I shall instead use the Heisenberg representation, in which averages are taken with the initial state $|\Delta \chi(0)\rangle$ while the observables become time dependent, to develop an evolution equation for the random hydrodynamic observables $\langle\boldsymbol{A}(t)|$. The mean of that equation (its contraction with $|\Delta \chi(0)\rangle$ ) reduces to the usual fluid equations, but the random Langevin equation also contains fluctuating forces, analogous to the Langevin theory for classical Brownian motion.

\footnotetext{
${ }^{29}$ The use of generalized Langevin equations in the context of statistical turbulence theory is reviewed by Krommes (2002).
} 


\subsection{Heisenberg versus Schrödinger representations}

While the Heisenberg representation is familiar from quantum mechanics, there are some technical differences in the present application that need to be appreciated; therefore, I digress for a brief review. In quantum mechanics, the Schrödinger equation

$$
\mathrm{i} \hbar \partial_{t} \psi=\mathrm{H} \psi
$$

can be written as

$$
\partial_{t} \psi=-\mathrm{i} \mathcal{L} \psi,
$$

where $\mathcal{L} \doteq \mathrm{H} / \hbar$. For time independent $\mathrm{H}$, the solution is given by

$$
\psi(t)=\mathrm{G}(t) \psi(0),
$$

where $\mathrm{G}(t) \doteq \mathrm{e}^{-\mathrm{i} \mathcal{L} t}$. Because $\mathrm{H}$ is self-adjoint with respect to the usual complex-valued scalar product, $\mathrm{G}$ is a unitary operator: $\mathrm{GG}^{\dagger}=1$.

In statistical mechanics, the $N$-particle $\operatorname{PDF} P_{N}(\Gamma, t)$, where $\Gamma$ is the set of all phase-space coordinates, obeys the Liouville equation

$$
\partial_{t} P_{N}(\Gamma, t)=-\mathrm{i} \mathcal{L} P_{N},
$$

where $\mathcal{L}$ is the Liouville operator. Thus, the state evolves as

$$
P_{N}(\Gamma, t)=\mathrm{e}^{-\mathrm{i} \mathcal{L} t} P_{N}(\Gamma, 0) .
$$

Since $\mathcal{L}$ is anti-self-adjoint with respect to a real-valued scalar product, time dependence can be transferred to observables (functions of $\Gamma$ that are to be averaged) according to

$$
\begin{aligned}
\langle A(\Gamma)\rangle & \doteq \int \mathrm{d} \Gamma A(\Gamma) P_{N}(\Gamma, t) \\
& =\int \mathrm{d} \Gamma A(\Gamma) \mathrm{e}^{-\mathrm{i} \mathcal{L} t} P_{N}(\Gamma, 0) \\
& =\int \mathrm{d} \Gamma\left[\mathrm{e}^{\mathrm{i} \mathcal{L} t} A(\Gamma)\right] P_{N}(\Gamma, 0) \\
& =\langle A(t ; \Gamma)\rangle_{0},
\end{aligned}
$$

where $A(t ; \Gamma) \doteq \mathrm{e}^{\mathrm{i} \mathcal{L} t} A(\Gamma)$ and the average is now with respect to the initial PDF. Thus, if the states are evolved with $\mathrm{G}(t) \doteq \mathrm{e}^{-\mathrm{i} \mathcal{L} t}$, the trajectories evolve with $\mathrm{G}(-t)$. This well-known result is a consequence of the fact that the microscopic dynamics is time reversible.

In the present situation governed by the linearized Landau kinetic equation, the state $\Delta \chi(\boldsymbol{v}, t)$ again evolves according to an equation of the form (4.2), where $\mathcal{L} \doteq \mathrm{L}_{v}+\mathrm{L}_{\mathrm{C}}+\mathrm{L}_{E}+\mathrm{L}_{\mathrm{M}}$ is given by (3.27). But $\mathcal{L}$ has no definite symmetry. The operators $\mathrm{L}_{v} \doteq \boldsymbol{k} \cdot \boldsymbol{v}$ and $\mathrm{L}_{\mathrm{C}} \doteq-\mathrm{i} \widehat{\mathrm{C}}$ are self-adjoint with respect to the natural (real-valued) scalar product, $\mathrm{L}_{\mathrm{M}}$ is readily shown to be anti-self-adjoint, and the electric-field operator $\mathrm{L}_{E} \propto|\boldsymbol{v}\rangle\langle 1|$ has no symmetry. Thus, the best one can do is to transfer the time dependence from the state to the observables according to

$$
\langle\boldsymbol{A} \mid \Delta \chi(t)\rangle=\langle\boldsymbol{A}(t) \mid \Delta \chi(0)\rangle,
$$


where

$$
\boldsymbol{A}(t) \doteq \mathrm{G}^{\dagger}(t) \boldsymbol{A}(0)
$$

with

$$
\mathrm{G}(t) \doteq \mathrm{e}^{-\mathrm{i} \mathcal{L} t}, \quad \mathrm{G}^{\dagger}(t) \doteq \mathrm{e}^{-\mathrm{i} \mathcal{L}^{\dagger} t}
$$

As a consistency check, note that the magnetic-field operator is a special case of the Liouville operator and possesses the same (anti)symmetry as is demonstrated by (4.6).

\subsection{Derivation of the generalized Langevin equation}

To derive the generalized Langevin equation, consider the time evolution of the hydrodynamic variables:

$$
\partial_{t}\langle\boldsymbol{A}(t)|=-\mathrm{i}\left\langle\mathcal{L}^{\dagger} \boldsymbol{A}(t)\right|=-\mathrm{i}\left\langle\mathcal{L}^{\dagger} \mathrm{G}^{\dagger} \boldsymbol{A}(0)\right|=-\mathrm{i}\left\langle\mathrm{G}^{\dagger} \mathcal{L}^{\dagger} \boldsymbol{A}(0)\right|,
$$

the last result following since $\mathcal{L}$ commutes with $\mathrm{G}$ (the latter being constructed from powers of $\mathcal{L}$ ). As in previous manipulations, this result will be manipulated by a judicious insertion of the identity $\mathrm{P}+\mathrm{Q}=1$. If that were done directly in the last form, virtually all of the symbols in the resulting expressions would be adorned with daggers. That could be avoided by working with the adjoint of (4.10). Alternatively, one can write formally

$$
-\mathrm{i}\left\langle\mathrm{G}(t)^{\dagger} \mathcal{L}^{\dagger} \boldsymbol{A}(0)\right|=-\mathrm{i}\langle\boldsymbol{A}(0)| \mathcal{L} \mathrm{G}(t),
$$

anticipating that this bra will ultimately be combined with the Heisenberg state $|\Delta \chi(0)\rangle$. Proceeding similarly to the manipulations in the Schrödinger-picture projection, I rewrite this as

$$
-\mathrm{i}\langle\boldsymbol{A}(0)| \mathcal{L} \mathrm{G}=-\mathrm{i}\langle\boldsymbol{A}(0)| \mathcal{L}(\mathrm{P}+\mathrm{Q}) \mathrm{G} .
$$

The P part of this becomes

$$
-\mathrm{i}\langle\boldsymbol{A}(0)|\mathcal{L}| \boldsymbol{A}(0)\rangle \cdot \boldsymbol{M}^{-1} \cdot\langle\boldsymbol{A}(0)| \mathrm{G}=-\mathrm{i} \boldsymbol{\Omega} \cdot\langle\boldsymbol{A}(t)| .
$$

For the Q part, Mori, Zwanzig, and others have shown that it is useful to express the final $\mathrm{G}$ in (4.12) in terms of the modified propagator $\mathrm{G}_{\mathrm{Q}}$ defined by (2.47). To do so, consider the Fourier transform of $\mathrm{G}(\tau)$,

$$
\widehat{G}(\omega)=\int_{0}^{\infty} \mathrm{d} \tau \mathrm{e}^{\mathrm{i} \omega \tau} \mathrm{e}^{-\mathrm{i} \mathcal{L} \tau}=[-\mathrm{i}(\omega-\mathcal{L}+\mathrm{i} \epsilon)]^{-1},
$$

and use the identity, valid for arbitrary non-commuting operators A and B (assuming that $\mathrm{A}^{-1}$ is defined),

$$
(\mathrm{A}+\mathrm{B})^{-1}=\mathrm{A}^{-1}-\mathrm{A}^{-1} \mathrm{~B}(\mathrm{~A}+\mathrm{B})^{-1},
$$

with

$$
\mathrm{A} \doteq-\mathrm{i}(\omega-\mathrm{Q} \mathcal{L} \mathrm{Q}+\mathrm{i} \epsilon), \quad \mathrm{B} \doteq \mathrm{i}(\mathcal{L}-\mathrm{Q} \mathcal{L} \mathrm{Q})
$$


Thus, ${ }^{30}$

$$
\widehat{G}(\omega)=\widehat{G}_{\mathrm{Q}}(\omega)-\widehat{G}_{\mathrm{Q}}(\omega)[\mathrm{i}(\mathcal{L}-\mathrm{Q} \mathcal{L} \mathrm{Q})] \widehat{G}(\omega)
$$

Now

$$
\mathcal{L}-\mathrm{Q} \mathcal{L Q}=\mathcal{L}-[(1-\mathrm{P}) \mathcal{L}(1-\mathrm{P})]=\mathrm{P} \mathcal{L}+\mathcal{L} \mathrm{P}-\mathrm{P} \mathcal{L} \mathrm{P}
$$

One requires $\mathrm{QG}$ for use in (4.12). Because $\mathrm{QG}_{\mathrm{Q}}=\mathrm{G}_{\mathrm{Q}} \mathrm{Q}$ and $\mathrm{QP}=0$, the first and last terms of (4.18) do not contribute to (4.17). Therefore, upon noting that $\mathrm{L}_{E}$ and $\mathrm{L}_{\mathrm{M}}$ do not contribute to $\mathrm{Q} \mathcal{L}$, one finds

$$
\mathrm{QG}=\mathrm{QG}_{\mathrm{Q}}-\mathrm{QG}_{\mathrm{Q}} \mathrm{iLPG} .
$$

Upon inserting the explicit form of $\mathrm{P}$ into (4.19), one can rewrite the last term of (4.12) as

$$
-\mathrm{i}\langle\boldsymbol{A}(0)| \mathrm{LQG}_{\mathrm{Q}}(t)-\left\langle\boldsymbol{A}(0)\left|\mathrm{LQG}_{\mathrm{Q}}(t) \mathrm{L}\right| \boldsymbol{A}^{\mathrm{T}}(0)\right\rangle \cdot \boldsymbol{M}^{-1} *\langle\boldsymbol{A}(t)|,
$$

where * denotes time convolution as well as dot product. The first term of (4.20) can be written as a random force $\langle f(t)|$, where

$$
\left|\boldsymbol{f}^{\mathrm{T}}(t)\right\rangle \doteq-\mathrm{iG}_{\mathrm{Q}}^{\dagger}(t) \mathrm{Q} \mathcal{L}^{\dagger}\left|\boldsymbol{A}^{\mathrm{T}}(0)\right\rangle=\mathrm{QG}_{\mathrm{Q}}^{\dagger}(t)\left|\dot{\boldsymbol{A}}^{\mathrm{T}}(0)\right\rangle .
$$

The last term of (4.20) can be written as

$$
-\left\langle\boldsymbol{A}(0)\left|\mathrm{LQG}_{\mathrm{Q}}(t) \mathrm{L}\right| \boldsymbol{A}^{\mathrm{T}}(0)\right\rangle \cdot \boldsymbol{M}^{-1} *\langle\boldsymbol{A}(t)|=-\boldsymbol{\Sigma} *\langle\boldsymbol{A}(t)|,
$$

where, upon recalling (4.21),

$$
\boldsymbol{\Sigma}(t) \doteq\left\langle\boldsymbol{f}(t) \boldsymbol{f}^{\mathrm{T}}(0)\right\rangle \cdot \boldsymbol{M}^{-1} .
$$

In summary, we have found the exact generalized Langevin equation ${ }^{31}$

$$
\partial_{t}\langle\boldsymbol{A}(t)|+\mathrm{i} \boldsymbol{\Omega} \cdot\langle\boldsymbol{A}(t)|+\int_{0}^{t} \mathrm{~d} \bar{\tau} \boldsymbol{\Sigma}(\bar{\tau}) \cdot\langle\boldsymbol{A}(t-\bar{\tau})|=\langle\boldsymbol{f}(t)| .
$$

${ }^{30}$ The inverse Fourier transform of (4.17) leads to

$$
G(\tau)=\mathrm{G}_{\mathrm{Q}}(\tau)-\int_{0}^{\tau} \mathrm{d} \bar{\tau} \mathrm{G}_{\mathrm{Q}}(\bar{\tau}) \mathrm{i}(\mathcal{L}-\mathrm{Q} \mathcal{L} \mathrm{Q}) G(\tau-\bar{\tau})
$$

This, or (4.15), is part of a family of similar identities. For example, one also has

$$
(\mathrm{A}+\mathrm{B})^{-1}=\mathrm{A}^{-1}-(\mathrm{A}+\mathrm{B})^{-1} \mathrm{BA}^{-1} .
$$

And instead of using $\mathrm{Q} \mathcal{L} \mathrm{Q}$ in the choices (4.16), one could choose $\mathrm{Q} \mathcal{L}$ instead. That leads to the identity

$$
\mathrm{e}^{-i \mathcal{L} \tau}=\mathrm{e}^{-\mathrm{iQ} \mathcal{L} \tau}-\int_{0}^{\tau} \mathrm{d} \bar{\tau} \mathrm{e}^{-\mathrm{i} \mathcal{L} \bar{\tau}} \mathrm{iP} \mathcal{L} \mathrm{e}^{-\mathrm{iQ} \mathcal{L}(\tau-\bar{\tau})} .
$$

Fox (1978) calls such identities disentanglement theorems and cites Feynman (1951). In the uses made of the modified propagator in practice, the final $\mathrm{Q}$ in $\mathrm{Q} \mathcal{L Q}$ is never necessary. (Equation (2.45) could have been written without the final $\mathrm{Q}$ before the second ket.) However, I prefer to work with the symmetrical construction $\mathrm{Q} \mathcal{L} \mathrm{Q}$.

${ }^{31}$ More commonly, this is written without the explicit bra notation. Given my definition of a bra, the content is identical; however, use of the bra emphasizes that the hydrodynamic observables are covectors, not vectors. 
To demonstrate compatibility with the previous results, one may apply $|\Delta \chi(0)\rangle$ to (4.24), thus performing the statistical average. One needs

$$
\langle\boldsymbol{f}(t) \mid \Delta \chi(0)\rangle=-\mathrm{i}\left\langle\boldsymbol{A}(0)\left|\mathrm{LG}_{\mathrm{Q}}(t) \mathrm{Q}\right| \Delta \chi(0)\right\rangle .
$$

This generates the same contribution to the $\mathrm{P}$ equation from the initial condition that one would have found by retaining the first term of (2.46). When the system is prepared in the hydrodynamic subspace, one has $|\mathrm{Q} \Delta \chi(0)\rangle=0$ and the contribution from the random force vanishes. The resulting equation,

$$
\left(\partial_{t}+\mathrm{i} \boldsymbol{\Omega}+\boldsymbol{\Sigma} *\right)\langle\boldsymbol{A}(t) \mid \Delta \chi(0)\rangle=0,
$$

is identical to that previously derived from the Schrödinger representation (see (2.48)).

\subsection{Fluctuating hydrodynamics and transport coefficients}

While (4.24) has the form of a generalized Langevin equation, it must not be assumed that it is always justifiable to treat $\langle\boldsymbol{f}(t)|$ as being white noise, as is often done in simple models (for example, see the discussion of the Brownian test particle in $\S$ G.3). The random noise involves the modified propagator $G_{Q}$, which encapsulates complicated details of the dynamics. Generalized Langevin equations can be derived for projections into essentially any subspace whatsoever, and the properties of $\langle\boldsymbol{f}|$ depend on the dimensionality of the subspace and the choice of variables $\boldsymbol{A}$ that is made. (For some important caveats relating to the choice of projection operators, see appendix G.) The issue is particularly clear when one follows Mori (1965) and projects the Liouville equation. Then (4.24) merely describes an exact rearrangement of the $N$-particle dynamics, with both (some) linear and nonlinear physics being buried in $\boldsymbol{f}$. Note that the precise way in which physics content is apportioned between $\boldsymbol{\Omega}$, $\boldsymbol{\Sigma}$ and $\boldsymbol{f}$ depends on the choice of the projection operator. In particular, for arbitrary $\mathrm{P}$ there is a part of $\boldsymbol{f}$ that lives partly in the hydrodynamic subspace and whose mean does not vanish. However, Zwanzig (2001, p. 156) shows that provided that one chooses $\mathrm{P}$ as I have done (using the standard scalar product), that mean vanishes to linear order. Furthermore, the specific choice of the hydrodynamic variables $\boldsymbol{A}$ that I have used to build P ensures that the long-wavelength limit of $\boldsymbol{\Sigma}$ is well behaved for the evolution of the conserved quantities. Thus, the exact generalized Langevin equation (4.24) is useful for the treatment of linear perturbations from thermal equilibrium, to which this paper is restricted. (In Part 2, I show how to generalize the procedure to include nonlinear effects.)

Indeed, for the standard hydrodynamic projection, several classical results for neutral fluids, as well as their extensions to magnetised plasmas, readily follow from the previous results. The topic of hydrodynamic fluctuations and their relation to transport coefficients has a long history that I shall not attempt to fully review here. In brief: Landau \& Lifshitz (1957, 1987) argued that the transport coefficients of a classical fluid are intimately related to the two-time correlation functions of certain fluctuating forces; for example, the thermal conductivity is related to the autocorrelation of a random heat flow. Kadanoff \& Martin (1963) stressed the importance of the double (ordered) limit $\lim _{\omega \rightarrow 0} \lim _{k \rightarrow 0}$ in extracting transport coefficients from certain response formulas. (See the discussion of the plateau phenomenon in §G.3.) The Landau-Lifshitz formulas were derived more systematically from kinetic theory by Bixon \& Zwanzig (1969), whose work was slightly generalized by Hinton (1970). A review article that provides useful background is by Fox (1978). 


\subsubsection{Transport coefficients as current-current correlations}

To tie those discussions of hydrodynamic fluctuations to the present formalism, compare (2.49) with formula (4.21), which defines the fluctuating force. One readily sees that

$$
\Sigma_{v}^{\mu}(\tau)=\left\langle f^{\mu}(\tau) f_{v}(0)\right\rangle .
$$

To obtain the Markovian transport matrix $\boldsymbol{\eta}_{v}^{\mu}$, (3.30), one takes $k, \omega \rightarrow 0$ in $\mathrm{G}_{\mathrm{Q}}$ (the order of the limits is immaterial). To illustrate, first consider the unmagnetised OCP. Then only $\mathrm{L}_{v}$ contributes to formulas (3.30) and (4.21). With $\nabla \rightarrow \mathrm{i} k$, one finds ${ }^{32}$

$$
\boldsymbol{\eta}_{v}^{\mu} \rightarrow \boldsymbol{k} \boldsymbol{k}:\left\langle A^{\mu}\left|\boldsymbol{v} \mathrm{Q} \widehat{\mathrm{C}}^{-1} \mathrm{Q} \boldsymbol{v}\right| A_{v}\right\rangle .
$$

If one writes $f^{\mu}=\nabla \cdot \widehat{\boldsymbol{J}}^{\mu}$ for generalized (subtracted) currents $\widehat{\boldsymbol{J}}^{\mu}$, then one has

$$
\boldsymbol{\eta}_{v}^{\mu}=\boldsymbol{k} \boldsymbol{k}:\left\langle\widehat{\boldsymbol{J}}^{\mu}\left|\widehat{\mathrm{C}}^{-1}\right| \widehat{\boldsymbol{J}}_{\nu}\right\rangle .
$$

By the symmetry of the unmagnetised system, the expectation must be proportional to the unit tensor,

$$
\left\langle\widehat{\boldsymbol{J}}^{\mu}\left|\widehat{\mathrm{C}}^{-1}\right| \widehat{\boldsymbol{J}}_{v}\right\rangle=D_{v}^{\mu} \boldsymbol{I},
$$

so one can obtain the generalized transport coefficients $D_{v}^{\mu}$ by

$$
D_{v}^{\mu}=k^{-2} \boldsymbol{\eta}_{v}^{\mu} .
$$

As an example, the thermal conductivity follows as

$$
\kappa=\left\langle\widehat{J}^{\mathrm{T}}\left|\widehat{\mathrm{C}}^{-1}\right| \widehat{J}_{T}\right\rangle,
$$

where

$$
\widehat{J}^{\mathrm{T}} \doteq\left(\frac{1}{2} \frac{v^{2}}{v_{\mathrm{t}}^{2}}-\frac{5}{2}\right) v_{z}
$$

Note that $(5 / 2) n T$ is the ideal-gas value of the enthalpy. The role of the enthalpy subtraction and the thermodynamic interpretation of $\widehat{J}^{\mathrm{T}}$ as a heat current is discussed by Kadanoff \& Martin (1963, p. 441).

\subsubsection{Fluctuating forces and collision-driven fluxes}

In the multispecies case, $\widehat{\mathrm{C}}$ also contributes to the $\mathrm{L}$ in $f^{\mu}$. That gives rise to a fluctuating friction force $\delta \boldsymbol{R}$ and a fluctuating thermal force $\delta \boldsymbol{q}$. The autocorrelation of $\delta \boldsymbol{R}$ leads to the non-hydrodynamic part of the friction force. The cross-correlation of $\delta \boldsymbol{R}$ and $\delta \boldsymbol{q}$ leads to the temperature-gradient-driven part of the momentum transfer and, by Onsager symmetry, to the flow-driven part of the heat flow.

The existence of all of these effects was well known to Braginskii, who interpreted the systematic Chapman-Enskog mathematics with simple physical pictures. Those arguments are entirely correct, and I have nothing to add to the physics. However, since Braginskii does not explicitly mention fluctuating forces in the sense of the

${ }^{32}$ For the $\mathrm{OCP}, \mathrm{Q} \widehat{\mathrm{C} Q}=\widehat{\mathrm{C}}$. 
present formalism, it is useful to understand the connection between the various approaches. As an example, consider the temperature-gradient contribution to the electron momentum transfer. This arises from

$$
\eta_{T}^{p}=\int_{0}^{\infty} \mathrm{d} \tau\left\langle f^{p}(\tau) f_{T}(0)\right\rangle=\int_{0}^{\infty} \mathrm{d} \tau\left\langle f^{p}(0)\left|\mathrm{G}_{\mathrm{Q}}(\tau)\right| f_{T}(0)\right\rangle
$$

when $f^{p}$ is evaluated with $\mathrm{L}_{\mathrm{C}}$ and $f_{T}$ is evaluated with $\mathrm{L}_{v}$.

The streaming contribution to the fluctuating heat flow is

$$
\begin{aligned}
\left|f_{T}(0)\right\rangle & =-\mathrm{iQL}_{v}\left|A_{T}(0)\right\rangle \\
& =-\mathrm{Q} v \cdot \nabla\left(\frac{3}{2} T\right)^{-1}\left|\frac{1}{2} \frac{v^{2}}{v_{\mathrm{t}}^{2}}-\frac{3}{2}\right\rangle \\
& =-\left(\frac{3}{2} T\right)^{-1}\left|v\left(\frac{1}{2} \frac{v^{2}}{v_{\mathrm{t}}^{2}}-\frac{5}{2}\right)\right\rangle \cdot \nabla .
\end{aligned}
$$

This describes the fact that a microscopic velocity stream carries with it the ideal-gas value of the enthalpy, which must be subtracted from the kinetic-energy flux to give the gradient-driven heat flow.

Next, one has

$$
\int_{0}^{\infty} \mathrm{d} \tau \lim _{k \rightarrow 0} \mathrm{G}_{\mathrm{Q}}(\tau)\left|f_{T}(0)\right\rangle=[\mathrm{Q}(\mathrm{i} \widehat{\mathrm{M}}+\widehat{\mathrm{C}}) \mathrm{Q}]^{-1}\left|f_{T}(0)\right\rangle .
$$

For the parallel physics, this states that the characteristic autocorrelation time of the fluctuations is the collision time, and it introduces the collisional mean free path $\lambda_{\mathrm{mfp}}$ as the characteristic characteristic length. In Braginskii's discussion, the macroscopic temperature profile is expanded in the small ratio $\lambda_{\mathrm{mfp}} / L_{\|}, L_{\|}$being a macroscopic parallel scale length. For perpendicular motions, the characteristic time scale is the gyroperiod, the characteristic extent of the interactions is the gyroradius $\rho \doteq v_{\mathrm{t}} /\left|\omega_{\mathrm{c}}\right|$, and in the limit of $v /\left|\omega_{\mathrm{c}}\right| \ll 1$ the net autocorrelation time is the gyroperiod reduced by the small ratio ${ }^{33} v /\left|\omega_{\mathrm{c}}\right|$, namely $\tau_{\mathrm{ac}}=\left(v /\left|\omega_{\mathrm{c}}\right|\right)\left|\omega_{\mathrm{c}}\right|^{-1}$.

The microscopic velocity stream mentioned above suffers the fluctuating friction force

$$
\left|f_{p}(0)\right\rangle=-\mathrm{Q} \widehat{\mathrm{C}}\left|A_{p}\right\rangle
$$

For the electrons, one has

$$
\widehat{\mathrm{C}}\left|A_{p}\right\rangle=\widehat{\mathrm{C}}_{e i}\left|T^{-1} \boldsymbol{v}\right\rangle .
$$

The distinction here between the contravariant component $A^{p}=m \boldsymbol{v}$ and covariant component $A_{p}=A^{p} / N_{p}=\boldsymbol{v} / T$ (see (2.28) and (2.29)) is important: the contravariant component contains a mass, whereas the covariant one does not. For the latter, this means that the conventional orderings in the mass ratio may be used, so $\widehat{\mathrm{C}}_{e i} \approx \widehat{\mathrm{C}}^{\text {Lor }}$ and

$$
\widehat{\mathrm{C}}_{e i}\left|T^{-1} \boldsymbol{v}\right\rangle \approx 2 T^{-1} v(v)|\boldsymbol{v}\rangle .
$$

${ }^{33}$ Gyration is non-dissipative. The ratio $v /\left|\omega_{\mathrm{c}}\right|$ is the fractional amount of dissipation per cycle. 
Sans the temperature factor, this can be interpreted as the velocity-dependent friction on a microscopic velocity stream, which is one of the principal ingredients in Braginskii's heuristic pictures (cf. Braginskii's discussion of his figure 1). Note that upon applying the $\mathrm{Q}$ that is required in (4.37), one obtains a ket that is orthogonal to $\langle\boldsymbol{v}|$ :

$$
\frac{1}{v_{\mathrm{te}}^{2} \tau_{e}}\left|\left[3 \sqrt{\frac{\pi}{2}}\left(\frac{v_{\mathrm{te}}}{v}\right)^{3}-1\right] \boldsymbol{v}\right\rangle
$$

This is, in fact, exactly the ket that multiplies $m \Delta \boldsymbol{u}$ in the second line of (3.35); it describes the non-hydrodynamic part of the flow-driven tail on the perturbed distribution function.

The net frictional effect on the microscopic heat flow is given by the crosscorrelation between the fluctuating friction force (4.40) and the fluctuating heat flow (4.36). It is easy to see that that correlation gives rise to the same matrix element calculated by Braginskii for the off-diagonal contribution to the heat flow.

\section{Discussion}

The purpose of this paper has been to describe the application of projection-operator methods to classical plasma transport for the special case of linear response and the Braginskii (or Navier-Stokes) transport coefficients.

In general, there are two routes to the derivation of irreversible transport coefficients: (i) first derive an irreversible kinetic equation from the reversible Liouville equation, then project into the hydrodynamic subspace; (ii) project the Liouville equation, then perform a $\Gamma$-space ensemble average in order to obtain the irreversible decay of correlation functions whose time integrals are the transport coefficients. In the present paper, route (i) was followed: projection was done on the (linearized) irreversible kinetic equation. (In Part 2, I shall instead follow route (ii).) It is useful to compare method (i) with the traditional Chapman-Enskog approach, which is reviewed in appendix A for the special case of the one-component plasma. Obviously, both that method and the projection-operator approach capture the same physics and make compatible predictions when their regimes of validity overlap. The traditional approach allows for a background zeroth-order flow, so it contains nonlinear advective derivatives. Those are absent in the linear-response formalism (developed via either projection operators or in any other way), where perturbations are made to an absolute Maxwellian distribution. However, both methods predict the same hydrodynamic fluxes to first order in the gradients.

At the level of linear response, the principal difference in the formalisms is the way in which the solvability constraints are satisfied. In the projection-operator method, the frequency operator $\mathrm{P} \mathcal{L} \mathrm{P}$ leads to the Euler part of the hydrodynamic equations, and use of the orthogonal projector Q in the correction terms replaces the traditional Chapman-Enskog substitution of the partial time derivatives in the Euler equations by spatial gradients (see (A 18a)). The projection-operator method provides an optimally concise representation of that algebra, which leads to the subtracted fluxes.

It must be emphasized, however, that the methods are equivalent only when the proper hydrodynamic projector is used. For classical transport, the natural projection operator is clear; it is built from the null eigenvectors of the linearized collision operator. However, one can project into any subspace whatsoever. Since the physics is invariant to the mathematical representation, the same results must ensue in the 
long-wavelength, low-frequency limit regardless of the choice of P. However, one must be extremely cautious because if the projection is chosen inaptly the Markovian approximation will not be satisfied. This issue is explored in $\S \S G .1-G .3$. However, a simple example given in $\S$ G.4 shows that provided that one projects at least into all of the null subspace of the collision operator, a higher-dimensional Markovian projection can also be used if one desires information additional to that contained in the natural transport equations.

For the purposes of introduction and simplicity, the calculations in this paper were restricted to linear perturbations and first-order dissipative processes, and they assumed the validity of the Landau collision operator. That is not always adequate; for example, it precludes consideration of convective amplification of locally unstable fluctuations (Kent \& Taylor 1969), even when the system is globally stable. Non-local wave-induced transport (Rosenbluth \& Liu 1976) is also omitted from the present calculations. A few further words on those topics are given in Part 2.

Furthermore, the various approximations made in this paper may obscure some deep conceptual issues and certain general properties of hydrodynamic transport theory. For example, the transport matrix $\eta$ was shown to be the infinite-time integral of the two-time correlation function of certain fluctuating forces - but as defined in this paper, those forces were not exact, being built from the Landau operator. In fact, a generalization of these techniques defines the true $\boldsymbol{\eta}$ in terms of a certain exact two-time correlation function. This is done in Part 2, where it is also shown how to recover the present results in the limit of weak coupling.

A related point is the possible existence and construction of an exact hydrodynamic invariant manifold embedded in the kinetic many-body system, as discussed by Gorban \& Karlin (2014). If that exists, it would be an attractor from which an appropriate projection leads to an certain set of exact hydrodynamic equations. For neutral fluids, this is apparently the case in the limit of vanishing Mach number and Knudsen number ${ }^{34}$ (Gorban \& Karlin 2014, and references therein), and this implies that the plasma equations that were developed in the present paper are likely to be good descriptions of physical reality. Calculation of Burnett effects (see Part 2) is the next step in the asymptotic construction of such a manifold. However, an invariant hydrodynamic manifold may not exist at all due to entanglement between the hydrodynamic and non-hydrodynamic modes. The pursuit of such issues goes well beyond the scope of this paper, but it is an interesting area for future research.

In conclusion, the projection-operator approach to the derivation of linearized fluid equations is intuitive and technically efficient. It embeds the classical plasma derivations of transport equations into more general and modern formulations of statistical dynamics. Although projection is a linear operation, the methodology is useful even for nonlinear response, as Brey et al. (1981) have shown. That topic is addressed in Part 2, where it is shown how to obtain nonlinear fluid equations and the second-order (Burnett) corrections to the classical transport coefficients. It should also be clear that the formalism is not restricted to classical transport; one can contemplate applications to neoclassical theory and to gyrokinetics (Krommes 2012), for example. Projection-operator methods should be in the toolbox of every serious plasma theorist.

\footnotetext{
${ }^{34}$ The Knudsen number, a measure of the strength of the gradients, is defined in appendix A.
} 


\section{Acknowledgements}

This paper is dedicated to Professor A. Kaufman, one of the pioneers of classical plasma transport theory, whose concise and beautiful technical approaches to the calculation of various linear and nonlinear plasma processes provided some of the inspirations for this work. I am grateful to G. Hammett for useful suggestions on the manuscript. This work was supported by the US Department of Energy Contract DE-AC02-09CH11466.

\section{Appendix A. The traditional Chapman-Enskog calculation for the one-component plasma}

It is instructive to compare the traditional Chapman-Enskog approach to hydrodynamic equations and transport coefficients with the projection-operator formalism described in the main text. In the present appendix, I present my own version of the traditional calculation for the simplest case of the unmagnetised one-component plasma (OCP). The procedure was described by Robinson \& Bernstein (1962). In essence, my discussion is little more than a transcription of their outline to the notation of the present paper, but I have also included some additional pedagogical content.

I assume that the plasma consists of discrete ions with a smooth neutralizing electron background. Then the relevant collision operator is the ion-ion Landau operator $\mathrm{C}_{i i}$; I shall subsequently drop the subscripts. That velocity-space operator conserves the densities of number, momentum and kinetic energy without the necessity for summation over species. The governing kinetic equation can be written as

$$
\frac{\mathrm{D} f}{\mathrm{D} t}=-\mathrm{C}[f],
$$

where $\mathrm{D} / \mathrm{D} t$ denotes the Vlasov operator defined in (2.9) and the brackets denote functional dependence.

I shall use the method of multiple time and space scales (Chow 2007, and references therein) and consider time variations slow with respect to the collision time and spatial variations much longer than the collision mean free path $\lambda_{\mathrm{mfp}}$, which is taken to be much smaller than the box size or characteristic gradient scale length $L$. I thus use an ordering parameter $\epsilon \doteq \lambda_{\text {mfp }} / L \ll 1$ ( $\epsilon$ is called the Knudsen number Kn) and assume that

$$
\nu^{-1} \partial_{t}=O(\epsilon), \quad \lambda_{\mathrm{mfp}} \nabla=O(\epsilon) .
$$

I also assume that the electric field is small enough that the entire left-hand side of (A 1) is small. The method then proceeds by asymptotically expanding (A 1) order by order in $\epsilon$, using the multiple-scale definitions $t_{n} \doteq \epsilon^{n} t$ and $\boldsymbol{x}_{n} \doteq \epsilon^{n} \boldsymbol{x}$. Although in principle one could carry this process through all orders, I shall proceed only far enough to calculate dissipative transport to first order in the gradients (i.e. to obtain the Navier-Stokes transport coefficients). Then three physically distinct orders of expansion are relevant:

(i) $O\left(\epsilon^{0}\right)$ : kinetic scales - irreversible phenomena related to $90^{\circ}$ collisions: $\omega / v=$ $O(1), k \lambda_{\mathrm{mfp}}=O(1)$. On the kinetic time scale, the distribution function relaxes to a local Maxwellian distribution. I shall assume that that process has gone to completion (i.e. that $\partial_{t_{0}}$ and $\nabla_{0}$ vanish or, equivalently, that (A 2) holds). 
(ii) $O\left(\epsilon^{1}\right)$ : transit scales - reversible phenomena related to particles free streaming across the distance $L$. The fundamental assumption is that $L / \lambda_{\mathrm{mfp}}=\epsilon^{-1} \gg 1$. The associated transit time scale is $t_{\text {transit }}=L / v_{\mathrm{t}}=\left(L / \lambda_{\mathrm{mfp}}\right)\left(\lambda_{\mathrm{mfp}} / v_{\mathrm{t}}\right)=\epsilon^{-1} v^{-1}$, one order longer than the kinetic time scale. ${ }^{35}$

(iii) $O\left(\epsilon^{2}\right)$ : transport scales - irreversible phenomena related to classical diffusion and dissipation. If $\mu$ is a spatial transport coefficient with the classical random-walk scaling $\mu \sim \lambda_{\mathrm{mfp}}^{2} \nu$, then the characteristic diffusion rate $\mu \nabla^{2} \sim \mu / L^{2}$ satisfies $\left(\mu / L^{2}\right) / \nu=\left(\lambda_{\mathrm{mfp}} / L\right)^{2}=\epsilon^{2}$. It is not really necessary to introduce an $x_{2}$ scale, but it is convenient to do so for purposes of symmetry in the asymptotic expansion.

In the multiple-scale formalism, it is assumed that time and space variations can be extended to independent variations on the multiple scales: $f(\boldsymbol{x}, t)=f\left(\boldsymbol{x}_{0}, \boldsymbol{x}_{1}, \boldsymbol{x}_{2}, \ldots\right.$, $\left.t_{0}, t_{1}, t_{2}, \ldots\right)$. Thus,

$$
\frac{\partial}{\partial t}=\frac{\partial}{\partial t_{0}}+\epsilon \frac{\partial}{\partial t_{1}}+\epsilon^{2} \frac{\partial}{\partial t_{2}}+\cdots,
$$

and similarly for $\partial / \partial \boldsymbol{x}$. The distribution function is also expanded according to $f=$ $\sum_{n=0}^{\infty} f_{n} \epsilon^{n}$. Because it is assumed that relaxation on the kinetic scale has already gone to completion, one drops $\mathrm{D} / \mathrm{D} t_{0}$. Then, through $O\left(\epsilon^{2}\right)$, one obtains

$$
\begin{aligned}
0 & =-\mathrm{C}\left[f_{0}\right], \\
\frac{\mathrm{D} f_{0}}{\mathrm{D} t_{1}} & =-\widehat{\mathrm{C}} f_{1}, \\
\frac{\mathrm{D} f_{0}}{\mathrm{D} t_{2}}+\frac{\mathrm{D} f_{1}}{\mathrm{D} t_{1}} & =-\left(\widehat{\mathrm{C}} f_{2}+\mathrm{C}\left[f_{1}, f_{1}\right]\right),
\end{aligned}
$$

where $\widehat{\mathrm{C}}$ is the linearized collision operator (see appendix $\mathrm{B}$ ) and the notation $\mathrm{C}\left[f_{1}, f_{1}\right]$ reflects the fact that the nonlinear Landau operator is actually a bilinear form. ${ }^{36}$

\section{A.1. The kinetic time scale}

The unique solution to (A $4 a$ ) is the local Maxwellian distribution

$$
f_{0}(\boldsymbol{x}, \boldsymbol{v}, t)=f_{\mathrm{IM}}\left(\boldsymbol{v} \mid \boldsymbol{x}_{1}, t_{1}, \boldsymbol{x}_{2}, t_{2}, \ldots\right),
$$

where

$$
f_{\mathrm{IM}}(\boldsymbol{v} \mid \boldsymbol{x}, t)=\left(\frac{n^{\prime}(\boldsymbol{x}, t)}{\bar{n}}\right)\left[\left[2 \pi v_{\mathrm{t}}^{\prime 2}(\boldsymbol{x}, t)\right]^{-3 / 2} \exp \left(-\frac{\left[\boldsymbol{v}-\boldsymbol{u}^{\prime}(\boldsymbol{x}, t)\right]^{2}}{2 v_{\mathrm{t}}^{\prime 2}(\boldsymbol{x}, t)}\right)\right],
$$

with $v_{\mathrm{t}}^{\prime 2}(\boldsymbol{x}, t) \doteq T^{\prime}(\boldsymbol{x}, t) / m$. Here the primed parameters specify the portions of the density, flow velocity and temperature that are carried by the local Maxwellian. Below I shall argue that they can be identified with the same quantities that are carried by the full distribution $f$, so I shall subsequently drop the primes. It is physically most instructive to isolate the density factor from the local Maxwellian. Thus, define $F_{0}$ as the factor in large brackets in (A 6), so

$$
f_{\mathrm{lM}}(\boldsymbol{v} \mid \boldsymbol{x}, t)=\left(\frac{n(\boldsymbol{x}, t)}{\bar{n}}\right) F_{0}(\boldsymbol{v} \mid \boldsymbol{x}, t) .
$$

Note that for the local Maxwellian the viscous stress $\boldsymbol{\pi}$ and the heat flow $\boldsymbol{q}$ vanish.

\footnotetext{
${ }^{35}$ With the definition $t_{1} \doteq \epsilon t$, it takes a time of $O\left(\epsilon^{-1}\right)$ to achieve an order-unity change in $t_{1}$; thus, the transit time scale is one order longer than the kinetic time scale.

${ }^{36}$ More generally, the Balescu-Lenard operator should be used; that is a more complicated nonlinear functional. The Balescu-Lenard operator is discussed in appendix 2:G.
} 


\section{A.2. The Transit time scale}

The viscous stress and heat flow are determined by the first-order correction to the local Maxwellian. Upon writing

$$
f_{1}=\left(\begin{array}{c}
n \\
\bar{n}
\end{array}\right) \chi F_{0}
$$

and using $F_{0}$ as the weight function in the natural scalar product defined by $(2.13 c)$, one can write the first-order correction equation (A $4 b$ ) as

$$
\left(\frac{n}{\bar{n}}\right) \widehat{\mathrm{C}}|\chi\rangle=-\frac{\mathrm{D}}{\mathrm{D} t_{1}}\left(\frac{n}{\frac{n}{n}}|1\rangle\right) .
$$

If $\widehat{\mathrm{C}}$ were positive-definite, a unique solution to this equation would be guaranteed. In fact, however, $\widehat{\mathrm{C}}$ is only positive-semidefinite since it has a five-dimensional null space associated with the conservation laws. Thus, either the solution does not exist or, if a certain solvability condition is satisfied, the solution exists but is not unique; this is the Fredholm alternative. Solvability is ensured by asserting that the right-hand side of (A 9) is orthogonal to the left null eigenvectors $\left\langle A^{\mu}\right|$, where the $A^{\mu}$ are defined in (2.25). Because $F_{0}$ is a Gaussian function of $\boldsymbol{w} \doteq \boldsymbol{v}-\boldsymbol{u}$, it is technically more convenient to use $\delta A^{\mu}$ instead of $A^{\mu}$, where $\delta A^{\mu}(\boldsymbol{v}) \doteq A^{\mu}(\boldsymbol{w})$. Thus, the solvability constraints are

$$
n\left\langle\delta A^{\mu}|\widehat{\mathrm{C}}| \chi\right\rangle=0=-\left\langle\delta A^{\mu}\left|\frac{\mathrm{D}}{\mathrm{D} t_{1}}\right| n\right\rangle,
$$

where the $\mathrm{D} / \mathrm{D} t_{1}$ acts on both the explicit $n$ as well as the space and velocity dependence of $F_{0}$ (which is hidden in the ket notation).

Those constraints determine the first-order evolution of the hydrodynamic variables $a^{\prime \mu}$. It is easy to see that the required averages are nothing but the hydrodynamic moments of the kinetic equation evaluated with first-order derivatives and with $\pi$ and $\boldsymbol{q}$ set to $\mathbf{0}$. These non-dissipative constraints are called the Euler equations. Thus, for example, the first of the five Euler equations is the continuity equation

$$
\frac{\partial n}{\partial t_{1}}+\nabla_{1} \cdot(n \boldsymbol{u})=0
$$

When the first-order Euler equations are satisfied, a solution to (A 9) is guaranteed. That solution is not unique, however, because an arbitrary linear superposition of the null eigenvectors can be added. Thus,

$$
|\chi\rangle=\sum_{\mu=1}^{5} \alpha^{\mu}\left|A_{\mu}\right\rangle+\left|\chi_{\perp}\right\rangle,
$$

where $\left\langle A^{\mu} \mid \chi_{\perp}\right\rangle=0$. To the extent that the $\alpha$ coefficients are non-zero, they specify the amounts of the hydrodynamic variables carried by the first-order distribution. However, there are no further constraints on the $\alpha$ values at this order, nor will any emerge at higher order. Thus, one is free to choose the $\alpha^{\mu}$ to vanish, and this freedom will persist through all orders. This means that one can arrange things such that all of the hydrodynamic variables are carried by the local Maxwellian; in other words, one may 
set $n^{\prime}=n, \boldsymbol{u}^{\prime}=\boldsymbol{u}$, and $T^{\prime}=T$. While this choice is not necessary, it is by far the most convenient.

With the constraints satisfied, one must now solve (A 9). The right-hand side of that equation can be simplified by carrying out the required partial time, space and velocity derivatives, then using the first-order Euler equations to replace the terms in $\partial / \partial_{t_{1}}$ by terms in $\nabla_{1}$. The algebra is straightforward. However, it is instructive to sketch it because there is an important lesson to be learned about the relation of this approach to the projection-operator method. Equation (A9) can be written as

$$
\widehat{\mathrm{C}}|\chi\rangle=-\left|\frac{\mathrm{D} \ln n}{\mathrm{D} t_{1}}\right\rangle-\left|\frac{\mathrm{D} \ln F_{0}}{\mathrm{D} t_{1}}\right\rangle \text {. }
$$

One has

$$
\frac{\mathrm{D} \ln n}{\mathrm{D} t_{1}}=\left(\partial_{t_{1}}+\boldsymbol{v} \cdot \nabla_{1}\right) \ln n
$$

Also, since

$$
\ln F_{0}=-\frac{w^{2}}{2 v_{\mathrm{t}}^{2}}-\frac{3}{2} \ln T+\text { const. }
$$

one has (upon temporarily dropping the 1 subscripts)

$$
\frac{\partial \ln F_{0}}{\partial t}=\frac{1}{v_{\mathrm{t}}^{2}} \boldsymbol{w} \cdot \frac{\partial \boldsymbol{u}}{\partial t}+\left(\frac{1}{2} \frac{w^{2}}{v_{\mathrm{t}}^{2}}-\frac{3}{2}\right) \frac{\partial \ln T}{\partial t}
$$

and similarly for $\boldsymbol{v} \cdot \nabla \ln F_{0}$. Upon using the Euler equations to replace the partial time derivatives, one finds

$$
\begin{aligned}
\partial_{t} \ln n= & -\boldsymbol{\nabla} \cdot \boldsymbol{u}+\boldsymbol{w} \cdot \nabla \ln n \\
\partial_{t} \ln F_{0}= & v_{t}^{-2} \boldsymbol{w} \cdot\left[-\boldsymbol{u} \cdot \nabla \boldsymbol{u}+(q / m) \boldsymbol{E}-(n m)^{-1}(T \nabla n+n \nabla T)\right] \\
& +\left(\frac{1}{2} \frac{w^{2}}{v_{\mathrm{t}}^{2}}-\frac{3}{2}\right) \frac{2}{3}(\boldsymbol{u} \cdot \nabla \ln T-\nabla \cdot \boldsymbol{u}) .
\end{aligned}
$$

In (A 13), some terms cancel and others combine as follows. One finds

$$
\begin{aligned}
\widehat{\mathrm{C}}|\chi\rangle= & -(\underbrace{-1}_{\partial_{t} \ln n}+\underbrace{1}_{\partial_{t} \ln T}) \boldsymbol{\nabla} \cdot \boldsymbol{u}|1\rangle-(\underbrace{1}_{\mathrm{L}_{v} \ln n}-\underbrace{1}_{\partial_{t} u})|\boldsymbol{w}\rangle \cdot \nabla \ln n \\
& -(\underbrace{-1}_{\mathrm{L}_{E} \ln F_{0}}+\underbrace{1}_{\partial_{t} u}) \frac{q}{T}|\boldsymbol{w}\rangle \cdot \boldsymbol{E} \\
& \left.-\frac{1}{v_{\mathrm{t}}^{2}}|\underbrace{\boldsymbol{w} \boldsymbol{w}}_{\mathrm{L}_{v} u}-\underbrace{\left.\frac{1}{3} w^{2} \boldsymbol{I}\right\rangle}_{\partial_{t} \ln T}: \boldsymbol{\nabla} \boldsymbol{u}-|(\underbrace{\frac{1}{2} \frac{w^{2}}{v_{\mathrm{t}}^{2}}-\frac{3}{2}}_{\mathrm{L}_{v} \ln F_{0}}-\underbrace{1}_{\partial_{t} u}) \boldsymbol{w}\right\rangle \cdot \nabla \ln T(\text { A } 18 a) \\
= & -\frac{1}{v_{\mathrm{t}}^{2}}\left|\boldsymbol{w} \boldsymbol{w}-\frac{1}{3} w^{2} \boldsymbol{I}\right\rangle: \boldsymbol{S}_{1}-\frac{1}{T}\left|\left(\frac{1}{\frac{1}{2}} \frac{w^{2}}{v_{\mathrm{t}}^{2}}-\frac{5}{2}\right) \boldsymbol{w}\right\rangle \cdot \nabla_{1} T,
\end{aligned}
$$

where $\boldsymbol{S}$ is the rate-of-strain tensor,

$$
\boldsymbol{S} \doteq \frac{1}{2}\left[(\nabla \boldsymbol{u})+(\nabla \boldsymbol{u})^{\mathrm{T}}\right]
$$


and the underbraces indicate the origins of the various terms. Note that in each of the pairwise combinations in (A 18a) the second term stems from a partial time derivative (i.e. from the enforcement of an Euler equation). This is the present algebra's way of ensuring that the right-hand side of (A 9) is orthogonal to the null eigenspace. In the projection-operator method, the same result is obtained by working with the orthogonal projector Q. In that approach, there is no explicit elimination of partial time derivatives; that is effectively done by the $\mathrm{P}$ term in $\mathrm{Q}=1-\mathrm{P}$ in the constructions QiLA. The physical reason for this subtraction is given in the paragraph following (2.69).

Because $\widehat{\mathrm{C}}$ is linear and the right-hand side is linear in the gradients, the solution to $(\mathrm{A} 18 b)$ can be determined by linear superposition to be $\chi=\chi_{u}+\chi_{T}$, where

$$
\chi_{u}=\boldsymbol{A}(\boldsymbol{w}): \boldsymbol{S}_{1}, \quad \chi_{T}=\boldsymbol{B}(\boldsymbol{w}) \cdot \nabla_{1} T,
$$

where

$$
\left.|\boldsymbol{A}\rangle=-\widehat{\mathrm{C}}^{-1} \frac{1}{v_{\mathrm{t}}^{2}}\left|\boldsymbol{w} \boldsymbol{w}-\frac{1}{3} w^{2} \boldsymbol{I}\right\rangle, \quad|\boldsymbol{B}\rangle=-\widehat{\mathrm{C}}^{-1} \frac{1}{T}\left|\left(\frac{1}{2} \frac{w^{2}}{v_{\mathrm{t}}^{2}}-\frac{5}{2}\right) \boldsymbol{w}\right\rangle . \quad \text { (A } 21 a, b\right)
$$

To evaluate these expressions, numerical work or approximate analytical methods (such as variational techniques (Robinson \& Bernstein 1962) or truncations of expansions in orthogonal polynomials (Braginskii 1965)) are required.

\section{A.3. The hydrodynamic regime}

One can now proceed to $O\left(\epsilon^{2}\right)$, where the effects of dissipation become apparent. Upon rearranging (A $4 c)$, one must solve

$$
\left.\left.\left(\frac{n}{\bar{n}}\right) \widehat{\mathrm{C}}\left|\chi_{2}\right\rangle=-\mid F_{0}^{-1} \mathrm{C}\left[f_{1}, f_{1}\right]\right)\right\rangle-\frac{\mathrm{D}}{\mathrm{D} t_{2}}\left|\left(\frac{n}{\bar{n}}\right) 1\right\rangle-\frac{\mathrm{D}}{\mathrm{D} t_{1}}\left|\left(\frac{n}{\bar{n}}\right) \chi_{1}\right\rangle .
$$

The solvability conditions are

$$
\begin{aligned}
\left\langle\bar{n} \delta A^{\mu}|\widehat{\mathrm{C}}| \chi_{2}\right\rangle=0= & -\left(\frac{\bar{n}}{n}\right)\left(\left\langle\bar{n} \delta A^{\mu} \mid F_{0}^{-1} \mathrm{C}\left[f_{1}, f_{1}\right]\right\rangle+\left\langle\delta A^{\mu}\left|\frac{\mathrm{D}}{\mathrm{D} t_{2}}\right| n\right\rangle\right. \\
& \left.+\left\langle\delta A^{\mu}\left|\frac{\mathrm{D}}{\mathrm{D} t_{1}}\right| n \chi_{1}\right\rangle\right) .
\end{aligned}
$$

The first term on the right-hand side vanishes because of the conservation properties of $\mathrm{C}^{37}$ The second term involves the same algebra that was done at first order and produces (sans a minus sign) the Euler moments in the $\boldsymbol{x}_{2}$ and $t_{2}$ variables. Finally, consider

$$
\begin{aligned}
\left\langle\delta A^{\mu}\left|\frac{\mathrm{D}}{\mathrm{D} t_{1}}\right| n \chi_{1}\right\rangle= & \left\langle\delta A^{\mu}\left|\partial_{t_{1}}\right| n \chi_{1}\right\rangle+\left\langle\delta A^{\mu}\left|\boldsymbol{v} \cdot \nabla_{1}\right| n \chi_{1}\right\rangle \\
& +\left\langle\delta A^{\mu}|\boldsymbol{E} \cdot \boldsymbol{\partial}| n \chi_{1}\right\rangle .
\end{aligned}
$$

${ }^{37}$ The $F_{0}^{-1}$ cancels against the hidden $F_{0}$ in the ket. 
The first term on the right-hand side vanishes because $\left|\chi_{1}\right\rangle$ has been constructed to be orthogonal to the null eigenfunctions. ${ }^{38}$ The last term also vanishes by orthogonality upon integration by parts. The middle term can be written as $\nabla_{1} \cdot \Gamma_{1}^{\mu}$, where

$$
\boldsymbol{\Gamma}_{1}^{\mu} \doteq n\left\langle\delta A^{\mu} \boldsymbol{v} \mid \chi_{1}\right\rangle=n\left\langle\delta A^{\mu} \boldsymbol{w} \mid \chi_{1}\right\rangle=(0 \pi \boldsymbol{q})_{1}^{\mathrm{T}} .
$$

Finally, add $\epsilon$ times the first-order solvability constraints (the first-order Euler equations) and $\epsilon^{2}$ times the second-order solvability constraints, and use $\epsilon \partial_{t_{1}}+\epsilon^{2} \partial_{t_{2}}$ $\approx \partial_{t}$ (and similarly for $\partial_{x}$ ). One thus reproduces the moment equations correct through second order and with explicit expressions for the fluxes:

$$
\boldsymbol{\pi}=-n m \boldsymbol{m}: \boldsymbol{S}, \quad \boldsymbol{q}=-n \boldsymbol{\kappa} \cdot \nabla T,
$$

where

$$
\begin{gathered}
\boldsymbol{m} \doteq \frac{1}{v_{\mathrm{t}}^{2}}\left\langle\boldsymbol{w} w-\frac{1}{3} w^{2} \boldsymbol{\imath}\left|\widehat{\mathrm{C}}^{-1}\right| \boldsymbol{w} \boldsymbol{w}-\frac{1}{3} w^{2} \boldsymbol{I}\right\rangle, \\
\boldsymbol{\kappa} \doteq\left\langle\left(\frac{1}{2} \frac{w^{2}}{v_{\mathrm{t}}^{2}}-\frac{5}{2}\right) \boldsymbol{w}\left|\widehat{\mathrm{C}}^{-1}\right|\left(\frac{1}{2} \frac{w^{2}}{v_{\mathrm{t}}^{2}}-\frac{5}{2}\right) \boldsymbol{w}\right\rangle .
\end{gathered}
$$

These tensors can be simplified by using symmetry considerations. The tensor $\boldsymbol{m}$ is symmetric and traceless in both the first and the last pair of its indices, and it depends on no preferred direction. The most general form of such a tensor is

$$
m_{i j k l}=a \delta_{i j} \delta_{k l}+\mu\left(\delta_{i l} \delta_{j k}+\delta_{i k} \delta_{j l}\right),
$$

where $a$ and $\mu$ are constants. Asserting the traceless condition leads to $a=-2 \mu / 3$. An expression for the scalar $\mu$ (kinematic viscosity) follows, for example, from $m_{1221}$ :

$$
\mu=v_{\mathrm{t}}^{-2}\left\langle w_{x} w_{y}\left|\widehat{\mathrm{C}}^{-1}\right| w_{x} w_{y}\right\rangle .
$$

Note that $\mu \sim v_{\mathrm{t}}^{2} / \nu$, which is the correct random-walk scaling for an unmagnetised transport coefficient. Contraction of $m_{i j k l}$ with $\boldsymbol{S}$ leads to

$$
\boldsymbol{\pi} \doteq-n m \mu \boldsymbol{W},
$$

where

$$
\boldsymbol{W} \doteq(\nabla \boldsymbol{u})+(\nabla \boldsymbol{u})^{\mathrm{T}}-\frac{2}{3}(\boldsymbol{\nabla} \cdot \boldsymbol{u}) \boldsymbol{l} .
$$

Similar considerations lead to $\kappa=\kappa \boldsymbol{l}$, where the thermal conductivity is

$$
\kappa=\left\langle\left(\frac{1}{2} \frac{w^{2}}{v_{t}^{2}}-\frac{5}{2}\right) w_{z}\left|\widehat{\mathrm{C}}^{-1}\right|\left(\frac{1}{2} \frac{w^{2}}{v_{t}^{2}}-\frac{5}{2}\right) w_{z}\right\rangle .
$$

This completes the review of the traditional Chapman-Enskog theory of the OCP, where dissipation is calculated just to first order in the gradients. In principle, one could proceed to higher order; at next order, the Burnett equations would emerge, but I shall not do so here. That calculation is treated in Part 2 using a generalization of the projection techniques described in the present paper.

The order-by-order Chapman-Enskog expansion constructs a one-particle distribution function that is slaved to the five hydrodynamic variables. Clearly, that cannot be the true distribution function of the kinetic system. Rather, the asymptotic procedure (attempts to) construct an invariant hydrodynamic manifold to which the system should quickly relax. For further discussion of this process, including the possibility that it may fail, please consult Gorban \& Karlin (2014, and references therein).

\footnotetext{
${ }^{38}$ One has $\left\langle\delta A^{\mu}\left|\partial_{t_{1}}\right| n \chi_{1}\right\rangle=\partial_{t_{1}}\left\langle\delta A^{\mu} \mid n \chi_{1}\right\rangle-\left\langle\left(\partial_{t_{1}} \delta A^{\mu}\right) \mid n \chi_{1}\right\rangle$. The first average vanishes directly by orthogonality. One has $\partial_{t_{1}} \delta A^{\mu}=\left(0,-m \partial_{t_{1}} \boldsymbol{u}^{\mathrm{T}},-m \boldsymbol{w} \cdot \partial_{t_{1}} \boldsymbol{u}-(3 / 2) \partial_{t_{1}} T\right)^{\mathrm{T}}$. The velocity dependence of the last result involves only 1 and $\boldsymbol{w}$, both of which are elements of the null space. Therefore, the second average vanishes as well.
} 


\section{Appendix B. The linearized Landau operator}

The Landau collision operator ${ }^{39}$ is

$$
C_{s \bar{s}}^{\mathrm{L}}[f]=-2 \pi(\bar{n} m)_{s}^{-1} \bar{S}_{s \bar{s}} \frac{\partial}{\partial \boldsymbol{v}} \cdot \int \mathrm{d} \overline{\boldsymbol{v}} \boldsymbol{U}(\boldsymbol{v}-\overline{\boldsymbol{v}}) \cdot\left(\frac{1}{m_{s}} \frac{\partial}{\partial \boldsymbol{v}}-\frac{1}{m_{\bar{s}}} \frac{\partial}{\partial \overline{\boldsymbol{v}}}\right) f_{s}(\boldsymbol{v}) f_{\bar{s}}(\overline{\boldsymbol{v}}),
$$

where

$$
\boldsymbol{U}(\boldsymbol{v}) \doteq v^{-1}(\boldsymbol{I}-\widehat{\boldsymbol{v}} \widehat{\boldsymbol{v}})
$$

and

$$
\bar{S}_{s \bar{s}} \doteq\left(\bar{n} q^{2}\right)_{s}\left(\bar{n} q^{2}\right)_{\bar{s}} \ln \Lambda_{s \bar{s}}
$$

(obviously symmetric in $s$ and $\bar{s}$ ). ${ }^{40}$ The proper definition of the Coulomb logarithm $\ln \Lambda$ is discussed by Krommes (2018a), who cites original references. Useful properties of $\boldsymbol{U}$, which is proportional to a projector into the direction perpendicular to its argument, are

$$
\boldsymbol{U}(\boldsymbol{v})=\frac{\partial^{2} v}{\partial \boldsymbol{v} \partial \boldsymbol{v}}, \quad \frac{\partial}{\partial \boldsymbol{v}} \cdot \boldsymbol{U}=-\frac{2 \boldsymbol{v}}{v^{3}} .
$$

$\mathrm{C}^{\mathrm{L}}$ is a bilinear operator on $f$, so it can be written as $\mathrm{C}[f, f]$ (I henceforth drop the $\mathrm{L}$ superscript for brevity). If one writes $f=(1+\Delta \chi) f_{\mathrm{M}}$, then the operator linearized around a Maxwellian involves

$$
\begin{aligned}
& \left(\frac{1}{m} \frac{\partial}{\partial \boldsymbol{v}}-\frac{1}{\bar{m}} \frac{\partial}{\partial \overline{\boldsymbol{v}}}\right)\left[(\Delta \chi+\Delta \bar{\chi}) f_{\mathrm{M}} \bar{f}_{\mathrm{M}}\right] \\
& \quad=\left(\frac{1}{m} \frac{\partial \Delta \chi}{\partial \boldsymbol{v}}-\frac{\boldsymbol{v}}{T}(\Delta \chi+\Delta \bar{\chi})\right) f_{\mathrm{M}} \bar{f}_{\mathrm{M}}-[(\boldsymbol{v}, s) \Leftrightarrow(\overline{\boldsymbol{v}}, \bar{s})] .
\end{aligned}
$$

The antisymmetrization introduces the relative velocity $\boldsymbol{v}-\overline{\boldsymbol{v}}$, which is annihilated by $\boldsymbol{U}(\boldsymbol{v}-\overline{\boldsymbol{v}})$. Thus, the linearized Landau operator is

$$
\begin{aligned}
\widehat{\mathrm{C}}|\Delta \chi\rangle= & -2 \pi(\bar{n} m)_{s}^{-1} \frac{\partial}{\partial \boldsymbol{v}} \cdot \sum_{\bar{s}} \bar{S}_{s \bar{s}} \int \mathrm{d} \overline{\boldsymbol{v}} \boldsymbol{U}(\boldsymbol{v}-\overline{\boldsymbol{v}}) \\
& \cdot\left(\left|\frac{1}{m_{s}} \frac{\partial \Delta \chi_{s}}{\partial \boldsymbol{v}}\right\rangle \bar{f}_{\mathrm{M}}-[(\boldsymbol{v}, s) \Leftrightarrow(\overline{\boldsymbol{v}}, \bar{s})]\right) .
\end{aligned}
$$

In practice, mass-ratio expansions of $\widehat{\mathrm{C}}$ are often useful. For electron-ion collisions, the ion integration velocity $\bar{v}$ is limited by the ion Maxwellian to be $O\left(v_{\mathrm{t} i}\right)$; thus, for typical electron velocities $\boldsymbol{v}$ one has $\boldsymbol{U}(\boldsymbol{v}-\overline{\boldsymbol{v}}) \approx \boldsymbol{U}(\boldsymbol{v})$ and

$$
\widehat{\mathrm{C}}_{e i}|\Delta \chi\rangle \approx-2 \pi(\bar{n} m)_{e}^{-1} \bar{S}_{e i} \frac{\partial}{\partial \boldsymbol{v}} \cdot \boldsymbol{U}\left(\boldsymbol{v}_{e}\right) \cdot\left(\frac{1}{m_{e}}\left|\frac{\partial \Delta \chi_{e}}{\partial \boldsymbol{v}_{e}}\right\rangle_{e}-|1\rangle_{e} \frac{1}{m_{i}}\left\langle 1 \mid \frac{\partial \Delta \chi_{i}}{\partial \boldsymbol{v}_{i}}\right\rangle_{i}\right) .
$$

Terms of $O\left(m_{e} / m_{i}\right)$ have been neglected in the electron term. The explicit $O\left(m_{i}^{-1}\right)$ ion term is not necessarily negligible because one does not yet know the size of

\footnotetext{
${ }^{39}$ A clear introduction to the Landau operator is given by Helander \& Sigmar (2002, chap. 3). A pedagogical compendium of useful properties of that operator is by Hazeltine (2006).

${ }^{40}$ The overline on $\bar{S}$ denotes evaluation with the mean density $\bar{n}$. An $S$ sans overline denotes evaluation with the full density $n$.
} 
the $\Delta \chi_{i}$ on which the operator will act. (Indeed, in some later projection operations one will need to insert $\Delta \chi$ values that are explicitly proportional to mass, so the mass dependence will cancel out in those cases.) When the ion term is in fact negligible, one obtains the Lorentz operator as usually defined:

$$
\widehat{\mathrm{C}}_{e i} \approx \widehat{\mathrm{C}}^{\mathrm{Lor}} \doteq v\left(\frac{v_{\mathrm{te}}^{3}}{v^{3}}\right) \boldsymbol{L}^{2}
$$

where $\boldsymbol{L}^{2}$ is the square of the angular momentum operator. ${ }^{41}$ The eigenfunctions of $\boldsymbol{L}^{2}$ are the spherical harmonics:

$$
\boldsymbol{L}^{2} Y_{l}^{m}(\theta, \phi)=l(l+1) Y_{l}^{m}
$$

with $Y_{l}^{m}(\theta, \phi) \doteq P_{l}^{m}(\cos \theta) \mathrm{e}^{\mathrm{i} m \phi}, P_{l}^{m}(x)$ being the associated Legendre functions of the first kind. The collision frequency $v$ is related to Braginskii's collision rate $\tau_{e}^{-1}$, defined in (B 13) below, by $v=(3 \sqrt{2 \pi} / 4) \tau_{e}^{-1}$.

From the definition (3.5b) and with the aid of integration by parts, (B 7) generates the electron momentum transfer $\boldsymbol{R} \approx \sum_{i} \boldsymbol{R}_{e i}$, where

$$
\boldsymbol{R}_{e i} \doteq-2 \pi \bar{S}_{e i}\left(\left\langle\boldsymbol{U} \cdot \frac{1}{m_{e}} \mid \frac{\partial \Delta \chi_{e}}{\partial \boldsymbol{v}_{e}}\right\rangle_{\mathrm{M} e}-\langle\boldsymbol{U}\rangle_{\mathrm{M} e} \cdot \frac{1}{m_{i}}\left\langle\frac{\partial \Delta \chi_{i}}{\partial \boldsymbol{v}_{i}}\right\rangle_{\mathrm{M} i}\right)
$$

A standard reference calculation assumes (illegitimately) that the distribution function is a local (shifted) Maxwellian: $f_{\mathrm{IM}} \doteq(n / \bar{n})\left(2 \pi v_{\mathrm{t}}^{2}\right)^{-3 / 2} \exp \left[-|\boldsymbol{v}-\boldsymbol{u}|^{2} / 2 v_{\mathrm{t}}^{2}\right] \approx f_{\mathrm{M}}(1+$ $\boldsymbol{v} \cdot \boldsymbol{u} / v_{\mathrm{t}}^{2}$ ) for $|u| / v_{\mathrm{t}} \ll 1$. (This is incorrect because of the formation of high-energy tails on $f$, as discussed and calculated later.) If $\Delta \chi_{s}=\boldsymbol{v} \cdot \boldsymbol{u}_{s} / v_{\mathrm{ts}}^{2}$ is inserted into (B 10), the explicit mass dependence cancels (a possibility that was noted above) and one finds with the aid of

$$
\langle\boldsymbol{U}\rangle_{\mathrm{Me}}=\frac{8 \pi}{3}(2 \pi)^{-3 / 2} v_{\mathrm{te}}^{-1} \boldsymbol{I}
$$

that

$$
\boldsymbol{R}_{e i} \approx-(m n)_{e} \tau_{e i}^{-1}\left(\boldsymbol{u}_{e}-\boldsymbol{u}_{i}\right)
$$

where

$$
\frac{1}{\tau_{e i}} \doteq \frac{4}{3} \sqrt{2 \pi} \frac{\left(q^{2}\right)_{e}\left(n q^{2}\right)_{i} \ln \Lambda_{e e}}{m_{e} T v_{\mathrm{t} e}}
$$

(cf. the definition (B 30) below of the generalized collision rate $v_{s s^{\prime}}$, which holds for arbitrary mass ratio). For the case of a single species of ions, Braginskii writes $\tau_{e i} \equiv \tau_{e}$.

For ion-electron collisions, one has

$$
\begin{aligned}
& \widehat{\mathrm{C}}_{i e}|\Delta \chi\rangle \approx-2 \pi(\bar{n} m)_{i}^{-1} \bar{S}_{i e} \frac{\partial}{\partial \boldsymbol{v}_{i}} \cdot \int \mathrm{d} \boldsymbol{v}_{e}\left(\boldsymbol{U}\left(\boldsymbol{v}_{e}\right)-\boldsymbol{v}_{i} \cdot \frac{\partial}{\partial \boldsymbol{v}_{e}} \boldsymbol{U}\left(\boldsymbol{v}_{e}\right)\right) \\
& \cdot\left(\frac{1}{m_{i}}\left|\frac{\partial \Delta \chi_{i}}{\partial \boldsymbol{v}_{i}}\right\rangle \bar{f}_{\mathrm{M}}\left(\boldsymbol{v}_{e}\right)-\frac{1}{m_{e}}\left|\frac{\partial \Delta \chi_{e}}{\partial \boldsymbol{v}_{e}}\right\rangle \bar{f}_{\mathrm{M}}\left(\boldsymbol{v}_{i}\right)\right)
\end{aligned}
$$

${ }^{41}$ Explicitly, in a spherical-polar $(v, \theta, \phi)$ coordinate system one has $\boldsymbol{L}^{2}=-\left[(\sin \theta)^{-1} \partial_{\theta} \sin \theta \partial_{\theta}+\right.$ $\left.\left(\sin ^{2} \theta\right)^{-1} \partial_{\phi}^{2}\right]$. See, for example, Gottfried (1966, p. 79). 


$$
\begin{aligned}
\approx & -2 \pi(\bar{n} m)_{i}^{-1} \bar{S}_{i e} \frac{\partial}{\partial \boldsymbol{v}_{i}} \cdot(\underbrace{\langle\boldsymbol{U}\rangle_{\mathrm{M} e} \cdot \frac{1}{m_{i}}\left|\frac{\partial \Delta \chi_{i}}{\partial \boldsymbol{v}_{i}}\right\rangle}_{\text {(a) }}+\underbrace{\left|\boldsymbol{v}_{i}\right\rangle \cdot \frac{1}{m_{e}}\left\langle\frac{\partial \boldsymbol{U}}{\partial \boldsymbol{v}_{e}} \cdot \mid \frac{\partial \Delta \chi_{e}}{\partial \boldsymbol{v}_{e}}\right\rangle}_{\text {(b) }} \\
& -\underbrace{\frac{1}{m_{e}}\left\langle\boldsymbol{U} \cdot \mid \frac{\partial \Delta \chi_{e}}{\partial \boldsymbol{v}_{e}}\right\rangle|1\rangle}_{\text {(c) }}{ }_{i}) .
\end{aligned}
$$

To understand the content of (B 14b), note that without linearization the ion-electron operator is approximately

$$
\mathrm{C}_{i e}[f] \approx-\frac{(m n)_{e}}{(m n)_{i}} \tau_{e}^{-1} \frac{\partial}{\partial \boldsymbol{v}_{i}} \cdot\left(\left(\boldsymbol{v}_{i}-\boldsymbol{u}_{i}\right) f_{i}+\frac{T_{e}}{m_{i}} \frac{\partial f_{i}}{\partial \boldsymbol{v}_{i}}\right)-\frac{1}{(m n)_{i}} \boldsymbol{R} \cdot \frac{\partial f_{i}}{\partial \boldsymbol{v}_{i}},
$$

where it was assumed that the electron distribution is a local Maxwellian. ${ }^{42}$ The linearization of this operator around absolute Maxwellians with equal electron and ion temperatures is

$$
\begin{aligned}
\Delta \mathrm{C}_{i e}[f]= & -\frac{(m n)_{e}}{(m n)_{i}} \tau_{e}^{-1} \frac{\partial}{\partial \boldsymbol{v}_{i}} \cdot[\underbrace{\boldsymbol{v}_{i} \Delta f_{i}}_{\left(\mathrm{a}^{\prime}\right)}+\underbrace{\frac{T}{m_{i}} \frac{\partial \Delta f_{i}}{\partial \boldsymbol{v}_{i}}}_{\left(\mathrm{b}^{\prime}\right)}-\underbrace{\Delta \boldsymbol{u}_{i} f_{i}}_{\left(\mathrm{c}^{\prime}\right)}-\underbrace{\boldsymbol{v}_{i}\left(\frac{\Delta T_{e}}{T}\right) f_{i}}_{\left(\mathrm{d}^{\prime}\right)}] \\
& -\underbrace{\frac{1}{(m n)_{i}} \frac{\partial}{\partial \boldsymbol{v}_{i}} \cdot\left(\Delta \boldsymbol{R} f_{i}\right)}_{\left(\mathrm{e}^{\prime}\right)} .
\end{aligned}
$$

For consistency, (B 14b) should reduce to this when $\Delta \chi_{e}$ is taken to be the perturbation of a local Maxwellian. That this is so is demonstrated in footnote $43 .{ }^{43}$

${ }^{42}$ If the approximation (B 12) is used for the momentum transfer, the operator assumes the appealing Fokker-Planck form

$$
\mathrm{C}_{i e}[f] \approx-\frac{(m n)_{e}}{(m n)_{i}} \tau_{e}^{-1} \frac{\partial}{\partial \boldsymbol{v}_{i}} \cdot\left(\left(\boldsymbol{v}_{i}-\boldsymbol{u}_{e}\right) f_{i}+\frac{T_{e}}{m_{i}} \frac{\partial f_{i}}{\partial \boldsymbol{v}_{i}}\right),
$$

appropriate for a test ion moving through a sea of electrons with mean flow $\boldsymbol{u}_{e}$.

43 Here I sketch how (B 14b) reduces to (B 16). Term (a) in (B 14b) can be rewritten by pulling the velocity derivative out of the ket according to

$$
\frac{1}{m_{i}}\left|\frac{\partial \Delta \chi_{i}}{\partial \boldsymbol{v}_{i}}\right\rangle=\frac{1}{m_{i}} \frac{\partial}{\partial \boldsymbol{v}_{i}}\left|\Delta \chi_{i}\right\rangle+\frac{\boldsymbol{v}_{i}}{T_{i}}\left|\Delta \chi_{i}\right\rangle \rightarrow \frac{1}{T}\left(\boldsymbol{v}_{i}\left|\Delta \chi_{i}\right\rangle+\frac{T}{m_{i}} \frac{\partial}{\partial \boldsymbol{v}_{i}}\left|\Delta \chi_{i}\right\rangle\right)
$$

for $T_{i}=T_{e}=T$, which when (B 11) is used reproduces terms $\left(\mathrm{a}^{\prime}\right)$ and $\left(\mathrm{b}^{\prime}\right)$. To evaluate term (b), note that for a local Maxwellian one has

$$
\begin{aligned}
\Delta \ln f_{e}=\Delta \chi_{e} & =\Delta\left[\ln \left(\frac{n_{e}}{\bar{n}}\right)-\frac{\left(\boldsymbol{v}-\boldsymbol{u}_{e}\right)^{2}}{2 v_{\mathrm{t} e}^{2}}-\frac{3}{2} \ln \left(2 \pi v_{\mathrm{t} e}^{2}\right)\right] \\
& =\frac{\Delta n_{e}}{\bar{n}}+\frac{\boldsymbol{v} \cdot \Delta \boldsymbol{u}_{e}}{v_{\mathrm{t} e}^{2}}+\left(\frac{1}{2} \frac{v^{2}}{v_{\mathrm{t} e}^{2}}-\frac{3}{2}\right) \frac{\Delta T_{e}}{T_{e}} .
\end{aligned}
$$

Then term (b) involves

$$
\left\langle\frac{\partial \boldsymbol{U}}{\partial \boldsymbol{v}_{e}} \cdot \mid \frac{\partial \Delta \chi_{e}}{\partial \boldsymbol{v}_{e}}\right\rangle=\left\langle\frac{\partial \boldsymbol{U}}{\partial \boldsymbol{v}_{e}} \cdot\left(\frac{\Delta \boldsymbol{u}_{e}}{v_{\mathrm{t} e}^{2}}+\frac{\boldsymbol{v}_{e}}{v_{\mathrm{t} e}^{2}} \frac{\Delta T_{e}}{T_{e}}\right)\right\rangle .
$$




\section{B.1. Properties of the linearized Landau operator}

The linearized Landau operator inherits the conservation laws of the full operator:

$$
\langle\boldsymbol{A}| \bar{n} \widehat{\mathrm{C}}=0,
$$

where the scalar product includes species summation (see (3.15)) and where

$$
\boldsymbol{A} \doteq\left(\begin{array}{lll}
1 & \boldsymbol{P}^{\mathrm{T}} & K^{\prime}
\end{array}\right)^{\mathrm{T}}
$$

This also follows directly from (B 6) upon integration by parts. This means that $\widehat{\mathrm{C}}$ has a five-dimensional null space, with $\langle\bar{n} \boldsymbol{A}|$ defining the left null eigenvectors. ${ }^{44}$ For further discussion of the spectrum of $\widehat{\mathrm{C}}$, see $\S \mathrm{B} .4$.

It can easily be shown from (B 6) that $\bar{n} \widehat{\mathrm{C}}$ is self-adjoint: ${ }^{45}$

$$
\langle\psi|\bar{n} \widehat{\mathrm{C}}| \chi\rangle=\langle\chi|\bar{n} \widehat{\mathrm{C}}| \psi\rangle
$$

Thus, upon taking the adjoint of (B 17), one finds that the right null eigenvectors are $|\boldsymbol{A}\rangle$ :

$$
\widehat{\mathrm{C}}|\boldsymbol{A}\rangle=0
$$

\section{B.2. Calculation of $\mathrm{PC} \mathrm{P}|\Delta \chi\rangle$}

To calculate $\mathrm{PC} P|\Delta \chi\rangle$, the dissipative part of the frequency matrix (see $(2.56 b)$ ), one first evaluates $\widehat{\mathrm{CP}}|\Delta \chi\rangle$, then applies $\mathrm{P}$ to that. Into the representation (B 6), one must replace $\Delta \chi$ by its projected value according to

$$
\Delta \chi_{s} \rightarrow 1 \frac{\Delta n_{s}}{\bar{n}_{s}}+\boldsymbol{v} \cdot \frac{1}{v_{\mathrm{ts}}^{2}} \Delta \boldsymbol{u}_{s}+K_{s}^{\prime} \frac{1}{T^{2}} \Delta T_{s},
$$

so

$$
\frac{1}{m_{s}} \frac{\partial \Delta \chi_{s}}{\partial \boldsymbol{v}}=\frac{1}{T} \Delta \boldsymbol{u}_{s}+\boldsymbol{v} \frac{1}{T^{2}} \Delta T_{s} .
$$

Upon integration by parts, the $\Delta \boldsymbol{u}_{e}$ term vanishes while the $\Delta T_{e}$ term reduces to $v_{\mathrm{t} e}^{-2}\langle\boldsymbol{U}\rangle\left(\Delta T_{e} / T_{e}\right)$ and leads to term $\left(\mathrm{d}^{\prime}\right)$. Finally, the momentum transfer is $\boldsymbol{R}=-\int \mathrm{d} \boldsymbol{v}_{e}(\bar{n} m \boldsymbol{v})_{e} \mathrm{C}_{e i}[f]$, where

$$
\mathrm{C}_{e i}[f] \approx-v v_{\mathrm{te}}^{3} \frac{\partial}{\partial \boldsymbol{v}_{e}} \cdot \boldsymbol{U}\left(\boldsymbol{v}_{e}-\boldsymbol{u}_{i}\right) \cdot \frac{\partial f_{e}}{\partial \boldsymbol{v}_{e}} .
$$

Then, after integration by parts,

$$
\Delta \boldsymbol{R}=-(\bar{n} m)_{e} v v_{\mathrm{te}}^{3} \int \mathrm{d} \boldsymbol{v}_{e}\left[\boldsymbol{u} \cdot \frac{\partial f_{e}}{\partial \boldsymbol{v}_{e}}-\boldsymbol{u}_{i} \cdot\left(\frac{\partial \boldsymbol{U}}{\partial \boldsymbol{v}_{e}}\right) \cdot \frac{\partial f_{e}}{\partial \boldsymbol{v}_{e}}\right] .
$$

The first term on the right-hand side is recognized as being proportional to term (c). It can thus be replaced by a term of $O(\Delta \boldsymbol{R})$ [term $\left.\left(\mathrm{e}^{\prime}\right)\right]$ and a term of $O\left(\Delta \boldsymbol{u}_{i}\right)$ [term $\left.\left(\mathrm{c}^{\prime}\right)\right]$. Thus, one has accounted for all of the terms in (B 16). The reader can check that all of the coefficients work out correctly.

${ }^{44}$ A proof that these are the only null eigenvectors is (essentially) given by Montgomery \& Tidman (1964, $\$ 7.2)$.

${ }^{45}$ The need for the density factor can be seen from the elementary estimate for the collision frequency $v_{s \bar{s}}$ of a test particle of species $s$ colliding with field particles of species $\bar{s}: v_{s \bar{s}} \sim \sigma_{s \bar{s}}\left|v_{s}-v_{\bar{s}}\right| n_{\bar{s}}$, where $\sigma$ is the scattering cross-section. This formula is not symmetric in the density; symmetry is restored by multiplying by $n_{s}$. 
For like-species collisions, this vanishes under the antisymmetrization; this is a manifestation of the conservation laws and the self-adjointness of $\bar{n} \widehat{\mathrm{C}}$. All of the remaining integrals can be performed for arbitrary mass ratio in terms of the error function and its derivative, but I shall not do so here; simplifications for small mass ratio are given in the next section. However, the general result for $\mathrm{PCP}|\Delta \chi\rangle$ is relatively simple. After integration by parts of $\mathrm{PC}=\mathrm{P} \partial_{v} \cdot \widehat{J}$, one must apply $|\boldsymbol{v}\rangle m T^{-1}\left\langle\boldsymbol{I}|+| K^{\prime}\right\rangle m N_{T}^{-1}\langle\boldsymbol{v}|$ to $\widehat{\boldsymbol{J}} \mathrm{P}|\Delta \chi\rangle$. It is clear from the formula (B 6) that one requires the integrals

$$
\int \mathrm{d} \boldsymbol{v} \mathrm{d} \overline{\boldsymbol{v}} f_{\mathrm{M}}(\boldsymbol{v}) f_{\mathrm{M}}(\overline{\boldsymbol{v}})\left(\begin{array}{c}
\boldsymbol{U}(\boldsymbol{v}-\overline{\boldsymbol{v}}) \\
\boldsymbol{v} \cdot \boldsymbol{U}(\boldsymbol{v}-\overline{\boldsymbol{v}}) \\
\boldsymbol{v} \cdot \boldsymbol{U}(\boldsymbol{v}-\overline{\boldsymbol{v}}) \cdot \boldsymbol{v}
\end{array}\right)
$$

These are best done by transforming to the relative and centre-of-mass coordinates

$$
\boldsymbol{w} \doteq \boldsymbol{v}-\overline{\boldsymbol{v}}, \quad \boldsymbol{W} \doteq(m \boldsymbol{v}+\bar{m} \overline{\boldsymbol{v}}) / M,
$$

where $M \doteq m_{s}+m_{\bar{s}}$, so

$$
\boldsymbol{v}=\boldsymbol{W}+\left(\frac{\bar{m}}{M}\right) \boldsymbol{w}, \quad \overline{\boldsymbol{v}}=\boldsymbol{W}-\left(\frac{m}{M}\right) \boldsymbol{w},
$$

and

$$
\int \mathrm{d} \boldsymbol{v} \mathrm{d} \overline{\boldsymbol{v}} f_{\mathrm{M}} \bar{f}_{\mathrm{M}} \ldots=\int \mathrm{d} \boldsymbol{W} \mathrm{d} \boldsymbol{w} \Phi_{M}(\boldsymbol{W}) \Phi_{\mu}(\boldsymbol{w}) \ldots,
$$

where $\Phi_{\widehat{\mu}}$ is a Maxwellian with variance defined by $\sigma^{2}=T / \widehat{\mu}$ for $\widehat{\mu}=\mu$ or $M$ with $\mu$ being the reduced mass, defined by

$$
\mu_{s \bar{s}}^{-1} \doteq m_{s}^{-1}+m_{\bar{s}}^{-1} .
$$

It is then easy to show that

$$
\int \mathrm{d} \boldsymbol{v} \mathrm{d} \overline{\boldsymbol{v}} f_{\mathrm{M}}(\boldsymbol{v}) f_{\mathrm{M}}(\overline{\boldsymbol{v}})\left(\begin{array}{c}
\boldsymbol{U}(\boldsymbol{v}-\overline{\boldsymbol{v}}) \\
\boldsymbol{v} \cdot \boldsymbol{U}(\boldsymbol{v}-\overline{\boldsymbol{v}}) \\
\boldsymbol{v} \cdot \boldsymbol{U}(\boldsymbol{v}-\overline{\boldsymbol{v}}) \cdot \boldsymbol{v}
\end{array}\right)=2\left(\frac{2}{\pi}\right)^{1 / 2} v_{\mathrm{t} \mu}^{-1}\left(\begin{array}{c}
(1 / 3) \boldsymbol{I} \\
\mathbf{0} \\
v_{\mathrm{t} M}^{2}
\end{array}\right) .
$$

The final result is

$$
\begin{aligned}
\mathrm{PC} P|\Delta \chi\rangle= & |\boldsymbol{v}\rangle N_{\boldsymbol{v}}^{-1} \cdot \sum_{s^{\prime}} v_{s s^{\prime}}\left(\Delta \boldsymbol{u}_{s^{\prime}}-\Delta \boldsymbol{u}_{s}\right) \\
& +\left|K^{\prime}\right\rangle N_{T}^{-1}\left[3 \sum_{s^{\prime}}\left(\frac{m_{s}}{M_{s s^{\prime}}}\right) v_{s s^{\prime}}\left(\Delta T_{s^{\prime}}-\Delta T_{s}\right)\right]
\end{aligned}
$$

where the generalized collision rate is defined by

$$
v_{s s^{\prime}} \doteq \frac{4}{3} \sqrt{2 \pi} \frac{q_{s}^{2}\left(n q^{2}\right)_{s^{\prime}}}{m_{s} T v_{\mathrm{t} \mu}} \ln \Lambda_{s s^{\prime}} .
$$




\section{B.3. Calculation of $\widehat{\mathrm{C}} \mathrm{P}|\Delta \chi\rangle$}

Ultimately, one requires $\widehat{\mathrm{C}} \mathrm{P}|\Delta \chi\rangle=(1-\mathrm{P}) \widehat{\mathrm{C}} \mathrm{P}|\Delta \chi\rangle$, so one needs the action of $\widehat{\mathrm{C}}$ on the hydrodynamic subspace. Major simplifications ensue for small mass ratio, which was assumed by Braginskii. For electron-ion collisions, one has to lowest order $\boldsymbol{U}(\boldsymbol{v}-$ $\overline{\boldsymbol{v}}) \approx \boldsymbol{U}(\boldsymbol{v})$, which projects into the direction perpendicular to $\boldsymbol{v}$. That removes the $\Delta T_{e}$ term of $\mathrm{P}|\Delta \chi\rangle$, and the integral over $\overline{\boldsymbol{v}}$ removes the $\Delta T_{i}$ term, which is odd in $\overline{\boldsymbol{v}}$. The second property of (B 4) can be used to simplify the divergence, and the result can be written in terms of the small- $m_{e}$ limit of the collision rate $v_{e i}$. Thus, for small mass ratio one finds

$$
\widehat{\mathrm{C}}_{e i} \mathrm{P}|\Delta \chi\rangle \approx 3 \sqrt{\frac{\pi}{2}} \frac{1}{v_{\mathrm{te}}^{2} \tau_{e i}}\left|\left(\frac{v_{\mathrm{te}}}{v}\right)^{3} \boldsymbol{v}\right\rangle \cdot \Delta \boldsymbol{u},
$$

where $\Delta \boldsymbol{u} \doteq \Delta \boldsymbol{u}_{e}-\Delta \boldsymbol{u}_{i}$, so the total flow-driven contribution to $\mathrm{Q} \widehat{\mathrm{C}}|\Delta \chi\rangle$ is

$$
\sum_{i} \frac{1}{v_{\mathrm{te}}^{2} \tau_{e i}}\left|\left[3 \sqrt{\frac{\pi}{2}}\left(\frac{v_{\mathrm{te}}}{v}\right)^{3}-1\right] \boldsymbol{v}\right\rangle \cdot \Delta \boldsymbol{u} .
$$

We shall see in $\S 3.3$ that this term behaves as a source that generates a contribution to $|\mathrm{Q} \Delta \chi\rangle$ (see (3.35)). The physics of this result is that under perturbation the electron distribution is not merely a shifted Maxwellian; a high-energy non-Maxwellian tail develops because of the inverse velocity dependence of the electron-ion collision frequency. Thus, in the unmagnetised plasma the approximation (B 10) is not correct; the true shape of the perturbed distribution determines, for example, the values of $\alpha$ in (3.9) and $\beta$ in (3.10).

For ion-electron collisions, on the other hand, it is a straightforward calculation using (B 14b) to show that to lowest order in the mass ratio $\widehat{\mathrm{C}}_{i e}|\Delta \chi\rangle$ lies entirely in the hydrodynamic subspace (i.e. that $\mathrm{QC} \mathrm{P}|\Delta \chi\rangle \approx 0$ ).

\section{B.4. The spectrum of the linearized Landau operator and its relation to the Markovian approximation}

Lewis (1967) has shown that $\widehat{\mathrm{C}}_{e}$ has a continuous spectrum except for the five discrete null eigenvalues. While many calculations involving $\widehat{\mathrm{C}}$ can be done without explicit reference to its spectrum, the spectral representation is the most direct way to argue for the validity of the Markovian approximation that is used in obtaining the standard form of the transport equations (for example, see (2.53)). What one needs to determine is whether a construction of the form

$$
|K(v, \tau)\rangle \doteq \mathrm{e}^{-\mathrm{Q} \widehat{\mathrm{C}} \tau}|\widehat{S}\rangle
$$

where $\mathrm{P}|\widehat{S}\rangle=0$, decays on the collisional time scale. This is easy to argue in the affirmative when the spectrum of $\widehat{\mathrm{C}}$ is discrete; however, a continuous spectrum introduces some subtleties. Therefore, I shall provide some discussion.

Note that since $|\widehat{S}\rangle=\mathrm{Q}|\widehat{S}\rangle$ by assumption, one has

$$
\mathrm{e}^{-\mathrm{Q} \widehat{\mathrm{CQ}} \tau}|\widehat{S}\rangle=\mathrm{e}^{-\mathrm{Q} \widehat{C} \tau}|\widehat{S}\rangle \text {. }
$$


For simplicity, assume that $\mathrm{P} \widehat{\mathrm{C}}=0$ (e.g. the case of self-collisions). Then $\mathrm{Q} \widehat{\mathrm{C}}=(1-$ $\mathrm{P}) \widehat{\mathrm{C}}=\widehat{\mathrm{C}}$. The simplest relevant example is the 1-D Fokker-Planck operator for a test particle of mass $M$ in a bath of temperature $T$ :

$$
\widehat{\mathrm{C}}_{i} f \doteq-\frac{\partial}{\partial v}\left(v v+D_{v} \frac{\partial}{\partial v}\right) f
$$

where the constant coefficients are related by the Einstein relation $D_{v}=(T / M) \nu$. With velocities being normalized to $v_{T} \doteq(T / M)^{1 / 2}$, (B 35) can be written as

$$
\widehat{\mathrm{C}}_{i}|\Delta \chi\rangle=-v \frac{\partial}{\partial v}\left|\frac{\partial \Delta \chi}{\partial v}\right\rangle
$$

where the implicit weight function is a Maxwellian with unit variance. From this representation, it can easily be seen that the operator is self-adjoint with respect to the standard scalar product. It is a 1-D model of the ion-electron collision operator $\widehat{\mathrm{C}}_{i e}$ with $M=m_{i}, T=T_{e}$, and $\boldsymbol{u}_{e}=\mathbf{0}$; it has a 1-D null eigenspace $|1\rangle$ associated with density conservation. One can readily verify that the eigenfunctions are the (probabilistic) Hermite polynomials: ${ }^{46}$

$$
\widehat{\mathrm{C}}_{i}\left|\mathrm{He}_{n}(v)\right\rangle=n v\left|\mathrm{He}_{n}(v)\right\rangle,
$$

where $n$ is a non-negative integer. Thus, $\widehat{\mathrm{C}}_{i}$ has a discrete spectrum, a property shared with the linearized Boltzmann operator (which has a 5-D null space). To determine the behaviour of (B 33) for this example operator, insert the completeness relation (resolution of the identity)

$$
\delta(v-\bar{v})=\sum_{n=0}^{\infty} \operatorname{He}_{n}(v) \frac{1}{n !} \operatorname{He}_{n}(\bar{v})\left(\frac{\mathrm{e}^{-v^{2} / 4}}{(2 \pi)^{1 / 4}}\right)\left(\frac{\mathrm{e}^{-\bar{v}^{2} / 4}}{(2 \pi)^{1 / 4}}\right)
$$

into (B 33):

$$
\begin{aligned}
|K(v, \tau)\rangle & =\mathrm{e}^{-\widehat{C}_{i} \tau} \sum_{n=0}^{\infty}\left|\mathrm{He}_{n}(v)\right\rangle \frac{1}{n !}\left\langle\operatorname{He}_{n} \mid \widehat{S}\right\rangle \\
& =\sum_{n=1}^{\infty} \mathrm{e}^{-n v \tau} \frac{1}{n !} \widehat{S}_{n}\left|\operatorname{He}_{n}(v)\right\rangle .
\end{aligned}
$$

(The $n=0$ term is excluded because $\mathrm{He}_{0}(v)=1$ and I have assumed that $\langle 1 \mid \widehat{S}\rangle=$ 0 .) This clearly decays on the collisional time scale, so there is no difficulty with justifying the Markovian approximation.

Now consider $\widehat{\mathrm{C}}=\widehat{\mathrm{C}}_{e e}$. Lewis (1967) showed that the solution of $\partial_{t}|f\rangle=-\widehat{\mathrm{C}}_{e e}|f\rangle$ has the continuous spectral representation (mostly using Lewis's notation)

$$
|f\rangle(\boldsymbol{c}, t)=\sum_{l, m} \int_{0}^{\infty} \mathrm{d} \rho\left(\lambda_{l}\right) \Psi_{l m}\left(\boldsymbol{c}, \lambda_{l}\right) \widetilde{F}_{l m}\left(\lambda_{l}\right) \mathrm{e}^{-\lambda_{l} \tau},
$$

\footnotetext{
${ }^{46}$ The first few one-dimensional probabilistic Hermite polynomials in a standard normalization such that $\int_{-\infty}^{\infty} \mathrm{d} v \mathrm{He}_{n}(v) \mathrm{He}_{n^{\prime}}(v)(2 \pi)^{-1 / 2} \mathrm{e}^{-v^{2} / 2} \equiv\left\langle\mathrm{He}_{n}(v) \mid \mathrm{He}_{n^{\prime}}(v)\right\rangle=n ! \delta_{n n^{\prime}}$ are $\mathrm{He}_{0}(v)=1, \mathrm{He}_{1}(v)=v, \mathrm{He}_{2}(v)=v^{2}-1$.
} 
where $\tau$ is a dimensionless time (normalized to an electron-electron collision time), $\rho$ is the spectral measure ${ }^{47} \mathrm{~d} \rho\left(\lambda_{l}\right) \doteq a_{l} \lambda_{l}^{-1 / 2} \mathrm{~d} \lambda_{l}$,

$$
\begin{aligned}
& \widetilde{F}_{l m}\left(\lambda_{l}\right) \doteq N_{l}^{-1} \int_{0}^{\infty} \mathrm{d} \boldsymbol{c} \Psi_{l m}^{*}\left(\boldsymbol{c}, \lambda_{l}\right) f(\boldsymbol{c}, 0), \\
& \Psi_{l m}\left(\boldsymbol{c}, \lambda_{l}\right) \doteq c^{-1} \mathrm{e}^{-c^{2} / 2} \psi_{l}(c, \lambda) Y_{l}^{m}(\theta, \phi),
\end{aligned}
$$

$c \doteq v /\left(\sqrt{2} v_{\mathrm{t}}\right)$, the $Y_{l}^{m}$ are the spherical harmonics normalized such that $\int \mathrm{d} \Omega Y_{l}^{m}\left(Y_{l^{\prime}}^{m^{\prime}}\right)^{*}$ $=N_{l} \delta_{l l^{\prime}} \delta_{m m^{\prime}}$, and the $\psi_{l}\left(c, \lambda_{l}\right)$ are the eigenfunctions that solve a particular linear, integro-differential, self-adjoint equation (Lewis's equation (20)) deduced from the collision operator linearized around a Maxwellian. Thus, functions $u(c)$ that are square-integrable on $(0, \infty)$ have the spectral (generalized Fourier) representation

$$
u(c)=\int_{0}^{\infty} \mathrm{d} \rho\left(\lambda_{l}\right) \psi_{l}\left(c, \lambda_{l}\right) \widetilde{u}\left(\lambda_{l}\right), \quad \widetilde{u}\left(\lambda_{l}\right)=\int_{0}^{\infty} \mathrm{d} c \psi_{l}^{*}\left(c, \lambda_{l}\right) u(c) .
$$

Given that the measure $\rho(\lambda)$ is continuous at $\lambda=0$, the point $\lambda=0$ can be excluded from the integration in (B 40). Thus, as Lewis states, the spectral representation can be shown to be complete for all perturbations conserving the densities of number, momentum, and kinetic energy. Furthermore, since $\lambda=0$ is absent, one finds from (B 40) that perturbations decay on the collisional time scale; ${ }^{48}$ thus, the Markovian approximation is justified (provided that the area under the curve is finite).

Note that the collisional decay described by (B 40) need not be exponential (unlike the case of a discrete spectrum) because it involves a continuous superposition of exponentials. A 1-D example is obtained by considering the operator $\widehat{\mathrm{C}} \rightarrow-\partial_{v}^{2}$, which is the velocity-space diffusion part of the operator (B 35) written in dimensionless variables with velocities normalized to $v_{T}$ and times normalized to $v^{-1}$. (Note that this operator is not self-adjoint with respect to the standard scalar product.) Unlike the operator (B 35), the diffusion operator has a continuous spectrum with plane-wave eigenfunctions $\exp (\mathrm{i} \Lambda v)$ and eigenvalues ${ }^{49} \lambda=\Lambda^{2}, \Lambda$ being the continuous Fourier variable conjugate to $v$. As an illustration, consider the specific initial condition $f(v, 0)=v\left[(2 \pi)^{-1 / 2} \mathrm{e}^{-v^{2} / 2}\right]$. It is straightforward to solve the diffusion equation by Fourier transformation to find

$$
f(v, \tau)=(1+2 \tau)^{-3 / 2} v \frac{1}{\sqrt{2 \pi}} \exp \left[-\frac{1}{2}\left(\frac{v^{2}}{1+2 \tau}\right)\right] .
$$

This function decays on the collisional time scale, although not exponentially, and $\int_{0}^{\infty} \mathrm{d} \tau f(v, \tau)$ is finite. ${ }^{50}$

${ }^{47}$ The proportionality constant $a_{l}$ is fixed by the chosen normalization of the 1-D eigenfunctions $\psi_{l}$.

${ }^{48}$ In general, one could contemplate non-physical initial conditions such that the decay was more complicated. However, the specific QLP terms arising in the projection-operator formalism involve benign, low-order moments of velocities scaled to $v_{\mathrm{t}}$. Their generalized Fourier transform (B 41b) thus involve $\lambda$ values that are $O(1)$ in dimensionless units, leading to time dependence that is $O(1)$ in $\tau$ (the collisional time scale).

${ }^{49}$ Notice that Lewis's measure $\mathrm{d} \rho \propto \lambda^{-1 / 2} \mathrm{~d} \lambda$ is proportional to $\mathrm{d} \Lambda$, so the decay in (B 40) is $\exp \left(-\Lambda^{2} \tau\right)$, just as for the diffusion operator. The physics is different, however, because for $\widehat{\mathrm{C}}_{e e}$ polarization drag is captured in the solution for $\psi_{l}(\lambda)$, which is not a simple plane wave (cf. the first unnumbered equation after Lewis's equation (32)).

${ }^{50}$ Regarding long-time tails that arise from superpositions of exponentials, a closely related phenomenon is the $\tau^{-d / 2}$ tail on the velocity correlation function that arises in classical kinetic theory from the spatial wavenumber superposition of slowly decaying hydrodynamic modes (Balescu 1975; Krommes \& Oberman 1976; Reichl 1998; Zwanzig 2001). A non-integrable $\tau^{-1}$ tail arises for $d=2$, giving rise to vexing issues relating to non-locality in 2-D hydrodynamics. 


\section{Appendix C. Covariant representation of the hydrodynamic projection}

Here I give justification and further discussion of the covariant representation of the hydrodynamic projection.

In the Dirac bra-ket notation, kets $|\cdot\rangle$ are conventionally interpreted as vectors, while bras $\langle\cdot|$ are interpreted as covectors. ${ }^{51}$ In a finite-dimensional vector space spanned by the basis vectors $\boldsymbol{e}_{i}$, vectors $\boldsymbol{v}$ are represented as $\boldsymbol{v}=v^{i} \boldsymbol{e}_{i}$, with the $v^{i}$ being called the contravariant components. Similarly, covectors $\boldsymbol{w}$ are represented as $\boldsymbol{w}=w_{i} \boldsymbol{e}^{i}$, where the $\boldsymbol{e}^{i}$ are the dual basis vectors and the $w_{i}$ are called the covariant components. In Dirac notation, one writes $|\boldsymbol{v}\rangle=v^{i}\left|\boldsymbol{e}_{i}\right\rangle \equiv v^{i}|i\rangle$, the underlying basis vectors being understood in the last notation. Similarly, $\langle\boldsymbol{w}|=w_{i}\left\langle\boldsymbol{e}^{i}\right| \equiv w_{i}\langle i|$.

It is useful to introduce an adjoint operation $\dagger$ that changes vectors into covectors (kets into bras) and vice versa:

$$
|\boldsymbol{v}\rangle^{\dagger}=\langle\boldsymbol{v}|, \quad \text { or }\left(v^{i}|i\rangle\right)^{\dagger}=v_{i}\langle i|
$$

Because in the application to hydrodynamics the natural scalar product is real valued, no complex conjugate is taken in the execution of the adjoint operation.

The components $A^{\mu}$ of $\boldsymbol{A}(\mu=1, \ldots, 5)$ are, in fact, the first few multidimensional Hermite polynomials. Multiplied by $f_{\mathrm{M}}$, the complete set of those polynomials spans the velocity space. Therefore, $\left\langle A^{\mu}\right|$ plays the role of a dual basis vector $\boldsymbol{e}^{\mu}$, consistent with the interpretation of a bra as a covector.

Define $M^{\mu \nu}=\left\langle A^{\mu} A^{\nu}\right\rangle$. The inverse of this matrix is naturally written with lower indices: $\left(\boldsymbol{M}^{-1}\right)_{\mu \nu}$. Interpret this inverse as a metric tensor $g_{\mu \nu}$ and lower indices according to $A_{\mu}=g_{\mu \nu} A^{\nu}$. The ket $\left|A_{\mu}\right\rangle$ is consistently interpreted as a basis vector $\boldsymbol{e}_{\mu}$.

Given this notation, one can define the (dimensionless and self-adjoint) hydrodynamic projector

$$
\mathrm{P}=\left|A_{\mu}\right\rangle\left\langle A^{\mu}|\equiv| \mu\right\rangle\langle\mu|
$$

The hydrodynamic projection of the state vector $|\Delta \chi\rangle$ is then

$$
\mathrm{P}|\Delta \chi\rangle=\left|A_{\mu}\right\rangle\left\langle A^{\mu} \mid \Delta \chi\right\rangle=\left|A_{\mu}\right\rangle \Delta a^{\mu},
$$

which defines the hydrodynamic variables $\Delta a^{\mu}$ as the contravariant components of a hydrodynamic vector.

The choice $g_{\mu \nu}=\left(\boldsymbol{M}^{-1}\right)_{\mu \nu}$ is a special case of the Weinhold metric (Weinhold 1975, 1976). The use of that metric in the context of a covariant representation of Onsager symmetries has been discussed by Krommes \& Hu (1993).

\section{Appendix D. An example of the calculation of some transport coefficients: classical electron heat flow}

Calculation of the classical electron heat flow in the limit of small ${ }^{52} \epsilon \doteq v_{e} /\left|\omega_{c e}\right|$ provides a good example of the use of the various formulas and gives insights that are

\footnotetext{
${ }^{51}$ For discussion of the distinction between vectors and covectors and of other related concepts, see a modern textbook on differential geometry such as Fecko (2006). The presentation by Misner, Thorne \& Wheeler (1973) is particularly pictorial and pedagogical.

${ }^{52}$ The classical transport coefficients in the small-collisionality limit were considered by Rosenbluth \& Kaufman (1958) and Kaufman (1960).
} 
not available from purely numerical calculations. I repeat for convenience Braginskii's result quoted in $\S 3.1:^{53}$

$$
\boldsymbol{q}_{\perp, e} \approx-4.66 n_{e} \kappa_{\perp, e} \nabla_{\perp} T_{e}+\frac{5}{2} n_{e}\left(\frac{c T_{e}}{e B}\right) \widehat{\boldsymbol{b}} \times \nabla T_{e}-\frac{3}{2} \frac{(n T)_{e}}{\omega_{\mathrm{ce}} \tau_{e}} \widehat{\boldsymbol{b}} \times \nabla \boldsymbol{u},
$$

where $\kappa_{\perp, e} \doteq \rho_{e}^{2} / \tau_{e}$ has the usual random-walk scaling. $\left(\rho_{e} \doteq v_{\text {te }} /\left|\omega_{\text {ce }}\right|\right.$ is the electron gyroradius.) I shall show that all of the numerical coefficients in this expression can be calculated analytically by approximately solving (3.35) for small $\epsilon$.

Linear superposition shows that the thermodynamic forces $\Delta \boldsymbol{W} \doteq \boldsymbol{W}[\Delta \boldsymbol{u}], \nabla \Delta T / T$, and $\Delta \boldsymbol{u}$ drive independent contributions to $|\mathrm{Q} \Delta \chi\rangle$. The $\Delta \boldsymbol{R}$ term (the last term of (3.35); see (3.36)) also behaves as a thermodynamic force, but its magnitude is determined as part of the solution. For the calculation of $\boldsymbol{q}$, defined by $(3.5 c)$, vector symmetry precludes a contribution from $\Delta W$. To calculate the $\nabla_{\perp} \Delta T$-driven heat flux, let $|\psi\rangle$ be the part of $|\mathrm{Q} \Delta \chi\rangle$ driven by $\nabla_{\perp} \Delta T / T$. Define $\widehat{t} \doteq \nabla \Delta T / T /(|\nabla \Delta T| / T)$ (this unit vector in the direction of the gradient is conventionally taken to lie in the $-\widehat{\boldsymbol{x}}$ direction), $\bar{\psi} \doteq \psi /(|\nabla \Delta T| / T)$, and $\overline{\boldsymbol{R}} \doteq \boldsymbol{R} /(|\nabla \Delta T| / T)$. Thus, with velocities normalized to $v_{\mathrm{te}}$, one must solve

$$
-(\mathrm{i} \widehat{M}+\widehat{\mathrm{C}})|\bar{\psi}\rangle=v_{\mathrm{te}}\left|\left(\frac{1}{2} v^{2}-\frac{5}{2}\right) \boldsymbol{v}_{\perp}\right\rangle \cdot \widehat{\boldsymbol{t}}+\frac{1}{\bar{n} T}\left|\boldsymbol{v}_{\perp}\right\rangle \cdot \Delta \overline{\boldsymbol{R}} .
$$

The most general solution is

$$
\bar{\psi}=a(v) \boldsymbol{v}_{\perp} \cdot \widehat{\boldsymbol{t}}+b(v) \boldsymbol{v}_{\perp} \times \widehat{\boldsymbol{t}} \cdot \widehat{\boldsymbol{b}},
$$

where $a(v)$ and $b(v)$ are unknown functions to be determined. Upon applying $\mathrm{i} \widehat{\mathrm{M}} \doteq \omega_{\mathrm{ce}} \partial / \partial \zeta$ to $\boldsymbol{v}_{\perp}=v_{\perp}(\sin \zeta,-\cos \zeta)^{\mathrm{T}}$, one finds $\mathrm{i} \widehat{\mathrm{M}} \boldsymbol{v}_{\perp}=\omega_{\mathrm{ce}} \boldsymbol{v} \times \widehat{\boldsymbol{b}}(\widehat{\mathrm{M}}$ rotates perpendicular velocity vectors by angle $\zeta$ ). Upon rearranging (D2) in anticipation of iteration in small $\epsilon$, one finds

$$
\left|a \boldsymbol{v}_{\perp}\right\rangle \times \widehat{\boldsymbol{t}} \cdot \widehat{\boldsymbol{b}}+\left|b \boldsymbol{v}_{\perp}\right\rangle \cdot \widehat{\boldsymbol{t}}=r_{e}\left|\left(\frac{1}{2} v^{2}-\frac{5}{2}\right) \boldsymbol{v}_{\perp}\right\rangle \cdot \widehat{\boldsymbol{t}}+\underbrace{\frac{1}{\bar{n} \omega_{\mathrm{ce}} T}\left|\boldsymbol{v}_{\perp}\right\rangle \cdot \Delta \overline{\boldsymbol{R}}[\bar{\psi}]}_{O(\epsilon)}+\underbrace{\frac{1}{\omega_{\mathrm{ce}}} \widehat{\mathbf{C}}|\bar{\psi}\rangle}_{O(\epsilon)},
$$

where $r_{e} \doteq v_{\mathrm{te}} / \omega_{\mathrm{ce}}$ (a negative quantity). Expand $a$ and $b$ in powers of $\epsilon$ (e.g. $a=$ $\left.\sum_{n=0}^{\infty} a_{n} \epsilon^{n}\right)$. One readily deduces that

$$
a_{0}=0, \quad b_{0}=\left(\frac{1}{2} v^{2}-\frac{5}{2}\right) r_{e} .
$$

At $O(\epsilon)$, one must satisfy

$$
\left|a_{1} \boldsymbol{v}_{\perp}\right\rangle \times \widehat{\boldsymbol{t}} \cdot \widehat{\boldsymbol{b}}+\left|b_{1} \boldsymbol{v}_{\perp}\right\rangle \cdot \widehat{\boldsymbol{t}}=\frac{1}{\bar{n} \omega_{\mathrm{c} e} T}\left|\boldsymbol{v}_{\perp}\right\rangle \cdot \Delta \overline{\boldsymbol{R}}\left[b_{0} \boldsymbol{v}_{\perp} \times \widehat{\boldsymbol{t}} \cdot \widehat{\boldsymbol{b}}\right]+\frac{1}{\omega_{\mathrm{ce}}} \widehat{\mathrm{C}}\left|b_{0} \boldsymbol{v}_{\perp}\right\rangle \times \widehat{\boldsymbol{t}} \cdot \widehat{\boldsymbol{b}} .
$$

From formula (3.37), one finds that the momentum transfer is given by $\Delta \overline{\boldsymbol{R}}=\boldsymbol{R} \times \widehat{\boldsymbol{t}} \cdot \widehat{\boldsymbol{b}}$, where

$$
\boldsymbol{R} \doteq-\left(\bar{n} m v_{\mathrm{t}}\right)_{e}\left\langle\boldsymbol{v}_{\perp}\left|\widehat{\mathrm{C}}^{\mathrm{Lor}}\right| b_{0} \boldsymbol{v}_{\perp}\right\rangle
$$

${ }^{53}$ As a reminder, Braginskii's gyrofrequencies are unsigned, whereas mine are signed. 
and $\widehat{\mathrm{C}}^{\mathrm{Lor}} \doteq v v^{-3} \boldsymbol{L}^{2}$. This integral can be evaluated by representing the 3-D velocity in a spherical coordinate system $(v, \theta, \phi)$ and recalling that the $(l=1, m=1)$ spherical harmonic is proportional to $\sin \theta \mathrm{e}^{\mathrm{i} \phi}$; thus, $\boldsymbol{L}^{2} \boldsymbol{v}_{\perp}=l(l+1) \boldsymbol{v}_{\perp}=2 \boldsymbol{v}_{\perp}$. The resulting integral is proportional to $\boldsymbol{I}_{\perp}$ by isotropy. With the result that the 3-D Maxwellian average of $v^{n}$ is ${ }^{54}$

$$
\left\langle v^{n}\right\rangle=\frac{4 \pi}{(2 \pi)^{3 / 2}} 2^{(n+1) / 2} \Gamma\left(\frac{n+3}{2}\right),
$$

the matrix element can be calculated. The final result is

$$
\Delta \boldsymbol{R}=\frac{3}{2} \frac{\bar{n}_{e}}{\omega_{\mathrm{ce}} \tau_{e}} \widehat{\boldsymbol{b}} \times \nabla \Delta T,
$$

which agrees with Braginskii's result for the perpendicular thermal force (see (3.10)).

Due to the rotational symmetry of $\widehat{\mathrm{C}}$, the last term of (D 6) is proportional to $\boldsymbol{v}_{\perp} \times$ $\widehat{\boldsymbol{t}} \cdot \widehat{\boldsymbol{b}}$. One therefore concludes that $b_{1}=0$. Contributions to $a_{1}$ arise from all of the $\Delta \overline{\boldsymbol{R}}$ and the $\widehat{\mathrm{C}}=\widehat{\mathrm{C}}_{e i}^{\mathrm{Lor}}+\widehat{\mathrm{C}}_{e e}$ terms. I shall omit the algebra relating to $\widehat{\mathrm{C}}$.

One can now undo the normalizations and proceed to calculate the heat flux

$$
\Delta \boldsymbol{q}=\left\langle\bar{n} K^{\prime}(v) \boldsymbol{v} \mid \psi\right\rangle
$$

$\left(K^{\prime}(v)\right.$ is defined by (2.24)) from

$$
\psi=-a v \cdot \nabla\left(\frac{\Delta T}{T}\right)+b \boldsymbol{v} \cdot \widehat{\boldsymbol{b}} \times \nabla\left(\frac{\Delta T}{T}\right) .
$$

The diamagnetic flux (in the direction orthogonal to the gradient) is ${ }^{55}$

$$
\begin{aligned}
\Delta \boldsymbol{q}_{*} & =-\left(\bar{n} T v_{\mathrm{t}}\right)_{e}\left\langle K^{\prime}(v) \boldsymbol{v} \mid b_{0}(v) \boldsymbol{v}\right\rangle \cdot \widehat{\boldsymbol{b}} \times \nabla\left(\frac{\Delta T}{T}\right) \\
& =-\frac{5}{2} \bar{n}_{e}\left(\frac{v_{\mathrm{te}}^{2}}{\omega_{\mathrm{ce}}}\right) \widehat{\boldsymbol{b}} \times \nabla \Delta T=\frac{5}{2} \bar{n}_{e}\left(\frac{c T_{e}}{e B}\right) \widehat{\boldsymbol{b}} \times \nabla \Delta T .
\end{aligned}
$$

Here I used the result (D 5) together with several instances of the formula (D 8); the answer agrees with (3.11b) and (D 1). The flux in the direction of the gradient has the form

$$
\Delta \boldsymbol{q}_{\perp}=-\bar{n} \kappa_{e} \nabla_{\perp} \Delta T
$$

where $\kappa_{e}=A \kappa_{\perp, e}$ with

$$
A \doteq \frac{1}{3}\left\langle\left(\frac{1}{2} v^{2}-\frac{3}{2}\right) v^{2} a_{1}(v)\right\rangle .
$$

Given the solution for $a_{1}(v)$, it is straightforward to work out the required matrix elements and find that

$$
A=\underbrace{\frac{3}{2}}_{\Delta \boldsymbol{R}}+\underbrace{\frac{7}{4}}_{\widehat{\mathrm{C}}_{e i}^{\text {Lor }}}+\underbrace{\sqrt{2}}_{\widehat{\mathrm{C}}_{e e}} \approx 4.66,
$$

${ }^{54}$ For even moments, formula (D 8) reduces to $\left\langle v^{2 n}\right\rangle=(2 n+1) !$ !.

${ }^{55}$ Matrix elements of the form $\langle G(v) v \boldsymbol{v}\rangle$ are by symmetry equal to $A \boldsymbol{I}$, where $A=\left\langle G(v) v^{2}\right\rangle / 3$. 
which reproduces the numerical coefficient in (D 1). A message from (D 15) is that all relevant thermodynamic forces, including self-collisions, contribute to the transport coefficient.

A similar calculation leads to the perpendicular heat flow driven by $\Delta \boldsymbol{u}$. A difference is that there is no zeroth-order term; the $\Delta \boldsymbol{u}$ source term is already $O(\epsilon)$. This implies that contributions from the momentum transfer and the explicit collisional correction (the analogues of the last two terms in (D4)) are $O\left(\epsilon^{2}\right)$ and can be neglected. Thus, with $\Delta \boldsymbol{u}=-|\Delta u| \widehat{\boldsymbol{x}}$, one finds

$$
b_{1}=-\frac{1}{v_{\mathrm{te}}^{2} \omega_{\mathrm{ce}} \tau_{e}}\left[3 \sqrt{\frac{\pi}{2}}\left(\frac{v_{\mathrm{te}}}{v}\right)^{3}-1\right] .
$$

The matrix element with $K^{\prime}$ is readily calculated, and one recovers the last term of (D 1).

A consequence of the fact that the $\Delta \boldsymbol{u}_{\perp}$-driven contributions to $\mathrm{Q} \boldsymbol{\psi}$ are $O(\epsilon)$ is that the perpendicular friction force is given dominantly by its value projected into the hydrodynamic subspace; see (3.29b). That is, the numerical coefficient in $\boldsymbol{R}_{\perp}=$ $-(\bar{n} m)_{e} \tau_{e}^{-1} \Delta \boldsymbol{u}$ is 1 . The physical explanation is that the rapid gyromotion rapidly restores the local Maxwellian in a time that is short compared to the time to form a high-energy tail.

\section{Appendix E. Decomposition of the stress tensor}

In the case of the one-component, weakly coupled plasma, the fourth-rank viscosity tensor $\boldsymbol{m}$ was shown in appendix A to depend on a single scalar coefficient $\mu$, the kinematic viscosity, as a consequence of symmetry; from (A 28), one has

$$
m_{i j k l}=\mu\left(\delta_{i l} \delta_{j k}+\delta_{i k} \delta_{j l}-\frac{2}{3} \delta_{i j} \delta_{k l}\right) .
$$

A background magnetic field $\boldsymbol{B}$ breaks the symmetry and the representation of $\boldsymbol{m}$ becomes more complicated. The most general form of $\boldsymbol{m}$ that is compatible with rotational symmetry in the plane perpendicular to $\boldsymbol{B}$ can be argued to depend on the three tensors

$$
\begin{gathered}
\boldsymbol{B} \doteq \widehat{\boldsymbol{b}} \widehat{\boldsymbol{b}}=b_{i} b_{j}=\left(\begin{array}{ccc}
0 & 0 & 0 \\
0 & 0 & 0 \\
0 & 0 & 1
\end{array}\right), \\
\boldsymbol{\delta}^{\perp} \doteq \boldsymbol{I}-\boldsymbol{B} \equiv \delta_{i j}^{\perp}=\left(\begin{array}{ccc}
1 & 0 & 0 \\
0 & 1 & 0 \\
0 & 0 & 0
\end{array}\right), \\
\boldsymbol{\beta} \doteq \widehat{\boldsymbol{b}} \times=-\epsilon_{i j 3}=\left(\begin{array}{ccc}
0 & -1 & 0 \\
1 & 0 & 0 \\
0 & 0 & 0
\end{array}\right),
\end{gathered}
$$

where the matrix forms are valid in a coordinate system in which $\boldsymbol{B}$ is locally in the $z$ direction.

The tensor $\boldsymbol{m}$ is symmetric and traceless in both of its first and last pairs of indices. Introduce a symmetrizing operation $\{\ldots\}$ that creates appropriately symmetric and traceless tensors out of its argument. Then the most general representation of $\boldsymbol{m}$ is 
$\boldsymbol{m}=\sum_{p=0}^{4} n \mu_{p} \boldsymbol{V}_{p}$, where, using Cartesian tensor notation and Braginskii's conventions for coefficients and signs,

$$
\begin{aligned}
& \boldsymbol{v}_{0} \doteq 3\{\boldsymbol{B} \boldsymbol{B}\}=3\left(B_{i j}-\frac{1}{3} \delta_{i j}\right)\left(B_{k j}-\frac{1}{3} \delta_{k l}\right), \\
& \boldsymbol{v}_{1} \doteq\left\{\boldsymbol{\delta}^{\perp} \boldsymbol{\delta}^{\perp}\right\}=\delta_{i k}^{\perp} \delta_{j l}^{\perp}+\delta_{i l}^{\perp} \delta_{j k}^{\perp}-\delta_{i j}^{\perp} \delta_{k l}^{\perp}, \\
& \boldsymbol{v}_{2} \doteq\left\{\boldsymbol{\delta}^{\perp} \boldsymbol{B}\right\}=\delta_{i k}^{\perp} B_{j l}+\delta_{i l}^{\perp} B_{j k}+B_{i k} \delta_{j l}^{\perp}+B_{i l} \delta_{j k}^{\perp}, \\
& \boldsymbol{v}_{3} \doteq-\frac{1}{2}\left\{\boldsymbol{\delta}^{\perp} \boldsymbol{\beta}\right\}=-\frac{1}{2}\left(\delta_{i k}^{\perp} \beta_{j l}+\delta_{i l}^{\perp} \beta_{j k}+\beta_{i k} \delta_{j l}^{\perp}+\beta_{i l} \delta_{j k}^{\perp}\right), \\
& \boldsymbol{V}_{4} \doteq-\{\boldsymbol{B} \boldsymbol{\beta}\}=-\left(B_{i k} \beta_{j l}+B_{i l} \beta_{j k}+\beta_{i k} B_{j l}+\beta_{i l} B_{j k}\right) .
\end{aligned}
$$

The multiplicative factors of 3 and $1 / 2$ are for later convenience. Note that the construction $\{\boldsymbol{\beta} \boldsymbol{\beta}\}$ is not independent because, for example, $\beta_{i j} \beta_{k l}=\epsilon_{i j 3} \epsilon_{k l 3}=$ $\delta_{i k}^{\perp} \delta_{j l}^{\perp}-\delta_{i l}^{\perp} \delta_{j k}^{\perp}$.

With these definitions, the $\boldsymbol{V}$ matrices obey the following properties: $\boldsymbol{V}_{p} \cdot \boldsymbol{V}_{p^{\prime}}=0$ for $p \neq p^{\prime}$,

$$
\begin{aligned}
& \boldsymbol{v}_{0}: \boldsymbol{v}_{0}=2 \boldsymbol{V}_{0}, \\
& \boldsymbol{V}_{1}: \boldsymbol{v}_{1}=2 \boldsymbol{V}_{1}, \\
& \boldsymbol{V}_{2}: \boldsymbol{v}_{2}=2 \boldsymbol{V}_{2}, \\
& \boldsymbol{V}_{3}: \boldsymbol{v}_{3}=-2 \boldsymbol{V}_{1}, \\
& \boldsymbol{V}_{4}: \boldsymbol{V}_{4}=-2 \boldsymbol{V}_{2},
\end{aligned}
$$

and

$$
\boldsymbol{V}_{0}+\boldsymbol{V}_{1}+\boldsymbol{V}_{2}=\{\boldsymbol{I} \boldsymbol{l}\}=\delta_{i l} \delta_{j k}+\delta_{i k} \delta_{j l}-\frac{2}{3} \delta_{i j} \delta_{k l},
$$

which is the kinematic part of $\boldsymbol{V}(\boldsymbol{B}=\mathbf{0})$ (see (E 1)).

To calculate $\boldsymbol{\pi}$, one needs to know the action of $\boldsymbol{V}_{i}$ on $\boldsymbol{\nabla} \boldsymbol{u}$. Because the $\boldsymbol{V}$ matrices are symmetric in their last two indices, this is equivalent to calculating $\boldsymbol{V}_{i}: \boldsymbol{S}$, where $\boldsymbol{S}$ is defined by (3.4c). Note that $\boldsymbol{\delta}^{\perp}$ and $\boldsymbol{B}$ are symmetric, whereas $\boldsymbol{\beta}$ is antisymmetric. Then

$$
\begin{aligned}
& \boldsymbol{V}_{0}: \boldsymbol{S}=3\left(\boldsymbol{B}-\frac{1}{3} \boldsymbol{l}\right)\left(\boldsymbol{B}-\frac{1}{3} \boldsymbol{I}\right): \boldsymbol{S}, \\
& \boldsymbol{V}_{1}: \boldsymbol{S}=2 \boldsymbol{S}_{\perp}-\boldsymbol{\delta}^{\perp} \operatorname{Tr} \boldsymbol{S}_{\perp}, \\
& \boldsymbol{V}_{2}: \boldsymbol{S}=2\left(\boldsymbol{\delta}^{\perp} \cdot \boldsymbol{S} \cdot \boldsymbol{B}+\boldsymbol{B} \cdot \boldsymbol{S} \cdot \boldsymbol{\delta}^{\perp}\right), \\
& \boldsymbol{V}_{3}: \boldsymbol{S}=\boldsymbol{\delta}^{\perp} \cdot \boldsymbol{S} \cdot \boldsymbol{\beta}-\boldsymbol{\beta} \cdot \boldsymbol{S} \cdot \boldsymbol{\delta}^{\perp}, \\
& \boldsymbol{V}_{4}: \boldsymbol{S}=2(\boldsymbol{B} \cdot \boldsymbol{S} \cdot \boldsymbol{\beta}-\boldsymbol{\beta} \cdot \boldsymbol{S} \cdot \boldsymbol{B}) .
\end{aligned}
$$

These are to be compared with Braginskii's equations (4.42). Instead of $\boldsymbol{S}$, he uses $\boldsymbol{W} \doteq 2(\boldsymbol{S}-(1 / 3) \boldsymbol{\nabla} \cdot \boldsymbol{u} \boldsymbol{l})$, so that $\boldsymbol{S}=(1 / 2) \boldsymbol{W}+(1 / 3) \boldsymbol{\nabla} \cdot \boldsymbol{u} \boldsymbol{l}$. The last term, proportional to the identity operator, does not contribute to $\boldsymbol{V}_{0}$ (because $\boldsymbol{S}$ is dotted with a traceless quantity), $\boldsymbol{V}_{2}$ (because $\boldsymbol{\delta}^{\perp}$ and $\boldsymbol{B}$ are orthogonal), or $\boldsymbol{V}_{3}$ and $\boldsymbol{V}_{4}$ (because of cancellations due to the antisymmetry). It contributes to $\boldsymbol{V}_{1}$ a term

$$
\left(\frac{2}{3} \nabla \cdot u-\nabla_{\perp} \cdot u_{\perp}\right) \delta^{\perp}=\left(\nabla_{\|} u_{\|}-\frac{1}{3} \nabla \cdot u\right) \delta^{\perp}=\frac{1}{2} W_{z z} \delta^{\perp} .
$$

Thus, one finds

$$
V_{0}: S=\frac{3}{2}\left(B-\frac{1}{3} I\right)\left(B-\frac{1}{3} I\right): W,
$$




$$
\begin{aligned}
& \boldsymbol{V}_{1}: \boldsymbol{S}=\boldsymbol{\delta}^{\perp} \cdot \boldsymbol{W} \cdot \boldsymbol{\delta}^{\perp}+\frac{1}{2}(\widehat{\boldsymbol{b}} \cdot \boldsymbol{W} \cdot \widehat{\boldsymbol{b}}) \boldsymbol{\delta}^{\perp}, \\
& \boldsymbol{V}_{2}: \boldsymbol{S}=\boldsymbol{\delta}^{\perp} \cdot \boldsymbol{W} \cdot \boldsymbol{B}+\boldsymbol{B} \cdot \boldsymbol{W} \cdot \boldsymbol{\delta}^{\perp}, \\
& \boldsymbol{V}_{3}: \boldsymbol{S}=\frac{1}{2}\left(\boldsymbol{\delta}^{\perp} \cdot \boldsymbol{W} \cdot \boldsymbol{\beta}-\boldsymbol{\beta} \cdot \boldsymbol{W} \cdot \boldsymbol{\delta}^{\perp}\right), \\
& \boldsymbol{V}_{4}: \boldsymbol{S}=\boldsymbol{B} \cdot \boldsymbol{W} \cdot \boldsymbol{\beta}-\boldsymbol{\beta} \cdot \boldsymbol{W} \cdot \boldsymbol{B} .
\end{aligned}
$$

These are equivalent to Braginskii's equations (4.42). Note that each of the constructions $\boldsymbol{V}_{i}: \boldsymbol{S}$ is symmetric, consistent with the overall symmetry of $\boldsymbol{\pi}$.

The magnetic-field scalings of the $\mu_{p}$ viscosities are

$$
\begin{aligned}
\mu_{0} & \sim B^{0} \quad \text { (parallel transport), } \\
\mu_{1}, \mu_{2} & \sim B^{-1} \quad \text { (non-dissipative gyroviscosities), } \\
\mu_{3}, \mu_{4} & \sim B^{-2} \quad \text { (cross-field transport). }
\end{aligned}
$$

It can be shown that $\mu_{1}\left(\omega_{\mathrm{c}}\right)=\mu_{2}\left(2 \omega_{\mathrm{c}}\right)$ and $\mu_{3}\left(\omega_{\mathrm{c}}\right)=\mu_{4}\left(2 \omega_{\mathrm{c}}\right)$. This is a consequence of (i) the fact that the spherical harmonics are eigenfunctions of both $\widehat{\mathrm{C}}$ and $i \widehat{\mathrm{M}}$, and (ii) the aptly chosen tensorial decompositions of the $\boldsymbol{V}_{p}$ matrices.

The non-dissipative gyroviscosities $\mu_{1}$ and $\mu_{2}$ emerge in collisionless Vlasov or gyrokinetic theory as well. In the collisionless limit, a Chapman-Enskog-truncated fluid description is inappropriate and gyrokinetics (Krommes 2012, and references therein) provides a much superior approach. For further discussion, see Belova (2001).

\section{Appendix F. Linear eigenmodes of the Braginskii equations}

After Fourier analysis in space, the linearized Braginskii equations can be written as

$$
\partial_{t} \boldsymbol{a}_{k}(t)=\boldsymbol{K}_{k} \cdot \boldsymbol{a}_{k},
$$

where $K$ is a $5 S \times 5 S$ square matrix. This leads to the $5 S$-dimensional eigenvalue problem

$$
\operatorname{det}(\boldsymbol{K}-\lambda \boldsymbol{l})=0
$$

where I have dropped the $\boldsymbol{k}$ labels for simplicity. In this appendix I discuss some aspects of the eigenmodes for first the unmagnetised one-component plasma (five eigenmodes; §F.1), then a two-species plasma (ten eigenmodes) in the two limits $\boldsymbol{B}=\mathbf{0}\left(\S\right.$ F.2) and $\epsilon \doteq v /\left|\omega_{\mathrm{c}}\right| \ll 1$ (§ F.3). (Only special cases are considered for the latter.) This knowledge is of intrinsic conceptual interest and is also useful for numerical work with the Braginskii equations. While one might be most interested in low-frequency phenomena, the Braginskii equations will not totally oblige; they contain high-frequency eigenvalues as well: Langmuir oscillations for $\boldsymbol{B}=\mathbf{0}$; hybrid oscillations for $\boldsymbol{B} \neq \mathbf{0}$. Those may limit the time step unless special care is used in the formulation of the numerical algorithm.

For simple cases, the eigenvalue calculations can be done by hand. However, for the most complicated situations the algebra becomes tedious. The determinant of a ten-dimensional matrix all of whose entries are unique contains $10 ! \approx 3.6 \times 10^{6}$ terms, each of which may be a complicated product. The matrices for the linearized Braginskii equations are fortunately not full; nevertheless, the fully expanded determinant for the $\boldsymbol{B} \neq \mathbf{0}$ case contains more than 2500 terms. Those are of various orders in the small parameters $\mu, \epsilon$, and $k^{2}$, and one is interested only in the dominant balances. Since diverse orderings are possible, machine-aided manipulations are useful in sorting out the details. ${ }^{56}$

\footnotetext{
${ }^{56}$ I used MATHEMATICA to guide and check the algebra. The basic operation is Det[], which returns the symbolic determinant of an $n$-dimensional square matrix; the result is an $n$ th-order polynomial in the
} 


\section{F.1. Eigenmodes of the unmagnetised one-component plasma}

The eigenmodes of the hydrodynamic equations of a neutral fluid are well known; however, they are non-trivially modified in the presence of the long-ranged Coulomb force. I shall illustrate that for the unmagnetised one-component plasma. These results are well known (Balescu 1975). However, lessons learned here generalize to the more complicated multispecies and magnetised problems, which I shall discuss in later sections.

The linearized continuity equation is

$$
\frac{\partial}{\partial t}\left(\frac{\Delta n}{n}\right)=-\mathrm{i} \boldsymbol{k} \cdot \Delta \boldsymbol{u}
$$

the linearized momentum equation is

$$
\frac{\partial \Delta \boldsymbol{u}}{\partial t}=\frac{q}{m} \Delta \boldsymbol{E}-(n m)^{-1} \mathrm{i} \boldsymbol{k} \Delta p-\mu k^{2} \Delta \boldsymbol{u}-\left(\frac{1}{3} \mu+\zeta\right) \boldsymbol{k} \boldsymbol{k} \cdot \Delta \boldsymbol{u}
$$

(here I allow for a bulk viscosity $\zeta$ ) with $\Delta \boldsymbol{E}=-\mathrm{i} k \Delta \phi$, and the linearized temperature equation is

$$
\frac{\partial}{\partial t}\left(\frac{\Delta T}{T}\right)=-\frac{2}{3} \mathrm{i} k \cdot \Delta \boldsymbol{u}-\frac{2}{3} \kappa k^{2}\left(\frac{\Delta T}{T}\right) .
$$

Decompose $\Delta \boldsymbol{u}$ into longitudinal and transverse components (with respect to $\boldsymbol{k}$ ):

$$
\Delta \boldsymbol{u}=\Delta \boldsymbol{u}^{\text {long }}+\Delta \boldsymbol{u}^{\text {trans }},
$$

where

$$
\Delta \boldsymbol{u}^{\text {long }} \doteq \widehat{\boldsymbol{k}} \widehat{\boldsymbol{k}} \cdot \Delta \boldsymbol{u}, \quad \Delta \boldsymbol{u}^{\text {trans }} \doteq(\boldsymbol{I}-\widehat{\boldsymbol{k}} \widehat{\boldsymbol{k}}) \cdot \Delta \boldsymbol{u}=\widehat{\boldsymbol{k}} \times(\Delta \boldsymbol{u} \times \widehat{\boldsymbol{k}}) .
$$

These components decouple according to

$$
\lambda \Delta \boldsymbol{u}^{\text {trans }}=-\mu k^{2} \Delta \boldsymbol{u}^{\text {trans }},
$$

which gives rise to two shear modes, each with $\lambda=-\mu k^{2}$, and

$$
\begin{gathered}
\lambda\left(\frac{\Delta n}{n}\right)=-\mathrm{i} k \Delta u^{\text {long }}, \\
\lambda \Delta u^{\text {long }}=-\mathrm{i} \omega_{\mathrm{p}}^{2} \frac{1}{k}\left(\frac{\Delta n}{n}\right)-\mathrm{i} v_{\mathrm{t}}^{2} k\left(\frac{\Delta n}{n}+\frac{\Delta T}{T}\right)-\left(\frac{4}{3} \mu+\zeta\right) k^{2} \Delta u^{\text {long }}, \\
\lambda\left(\frac{\Delta T}{T}\right)=-\frac{2}{3} \mathrm{i} k \Delta u^{\text {long }}-\frac{2}{3} \kappa k^{2}\left(\frac{\Delta T}{T}\right) .
\end{gathered}
$$

Upon defining $\chi_{\mu} \doteq 4 \mu / 3+\zeta$ and $\chi_{\mathrm{v}} \doteq \kappa / c_{\mathrm{v}}$, where $c_{\mathrm{v}} \doteq 3 / 2$ is the specific heat at constant volume for a three-dimensional ideal gas, (F9a)-(F9c) can be combined to

eigenvalue $\lambda \doteq-\mathrm{i} \omega$. CoefficientList [] extracts the coefficients of $\lambda$. Each coefficient in that list can be replaced by its dominant approximation with respect to a chosen variable by the user-defined module reducelist[list, var_]. That module expresses each term in list as a polynomial in var, then searches the coefficient list of that polynomial and returns the lowest-order term that is non-zero. Consecutive uses of that module for the various small parameters finally lead to relatively simple expressions for the characteristic polynomial, from which the dominant balances can be easily recognized by the use of Kruskal diagrams. 


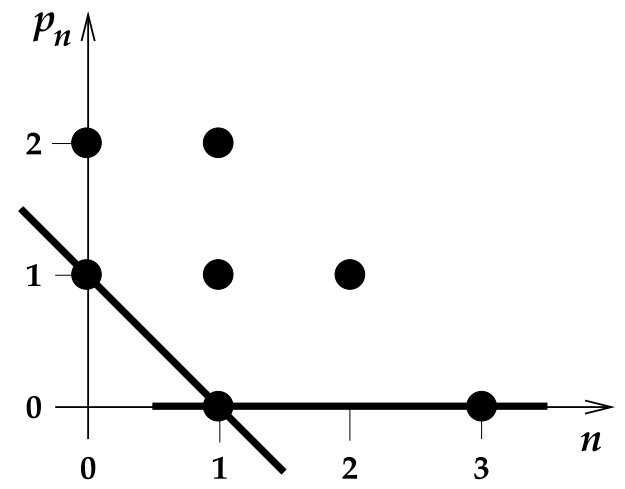

FIGURE 2. Kruskal diagram for the longitudinal modes of an unmagnetised onecomponent plasma (see (F10)), showing the balance between the terms in $\lambda^{0}$ and $\lambda^{1}$ (thermal-diffusion mode), and between $\lambda^{1}$ and $\lambda^{3}$ (plasma oscillations).

obtain the longitudinal dispersion relation

$$
\lambda^{3}+k^{2}\left(\chi_{\mu}+\chi_{\mathrm{v}}\right) \lambda^{2}+\left(\omega_{\mathrm{p}}^{2}+\frac{5}{3} k^{2} v_{\mathrm{t}}^{2}+\left(k^{2} \chi_{\mu}\right)\left(k^{2} \chi_{\mathrm{v}}\right)\right) \lambda+\left(\omega_{\mathrm{p}}^{2}+k^{2} v_{\mathrm{t}}^{2}\right)\left(k^{2} \chi_{\mathrm{v}}\right)=0 .
$$

Although a cubic equation has an explicit analytical solution, its interpretation is opaque in general. Fortunately, of most interest is the hydrodynamic limit $k \lambda_{\mathrm{mfp}} \rightarrow 0$. Upon noting that each of $\mu$ and $\kappa$ has the classical random-walk scaling $v_{\mathrm{t}}^{2} / \nu$, one can make (F 10) dimensionless by normalizing $\lambda$ to $v$, dividing by $v^{3}$, and introducing ${ }^{57} \delta \doteq\left(k v_{\mathrm{t}} / \nu\right)^{2}=\left(k \lambda_{\mathrm{mfp}}\right)^{2}$. One may treat $k^{2}$ as $O(\delta)$. For the roots of polynomials with small coefficients, an efficient and pictorial way of analysing the dominant balances is to use a Kruskal diagram (Kruskal 1965) in which the terms in the polynomial populate a 2-D lattice whose abscissa measures the powers of $\lambda$ and whose ordinate measures the powers of $\delta$. Dominant balances are found by bringing up lines from below until they rest on populated points. The Kruskal diagram for (F10) is shown in figure 2. The balance between the terms in $\lambda^{1}$ and $\lambda^{0}$ (I shall call that the 1-0 balance) signifies a thermal-diffusion mode with

$$
\lambda \approx-k^{2} \chi_{\mathrm{v}}=-k^{2} \kappa / c_{\mathrm{v}}
$$

The 3-1 balance leads to

$$
\lambda^{2}=\omega_{\mathrm{p}}^{2}+\frac{5}{3} k^{2} v_{\mathrm{t}}^{2}+O\left(\epsilon^{2}\right) .
$$

These are obviously plasma oscillations with real mode frequency $\Omega_{k} \approx \pm \omega_{\mathrm{p}}$, but with a thermal correction that is incorrect in the weakly coupled limit; the proper coefficient (which follows from collisionless kinetic theory) is 3 rather than $5 / 3$. The error arises because in the limit of weak coupling these modes do not satisfy the Markovian requirement $\left|\Omega_{k}\right| \ll v$, so one should not be taking the $\omega=0$ limit of the projection formalism. ${ }^{58}$ As is well known, the prediction (F12) is easily rationalized on physical grounds: $5 / 3$ is the ratio of specific heats $c_{\mathrm{p}} / c_{\mathrm{v}}=(d+2) / d$ of a

\footnotetext{
${ }^{57}$ This is the square of the Chapman-Enskog expansion parameter used in appendix A.

${ }^{58}$ This point is well known in related contexts. For example, high-frequency conductivity has been treated thoroughly by Dawson \& Oberman (1962) and Dawson (1968).
} 


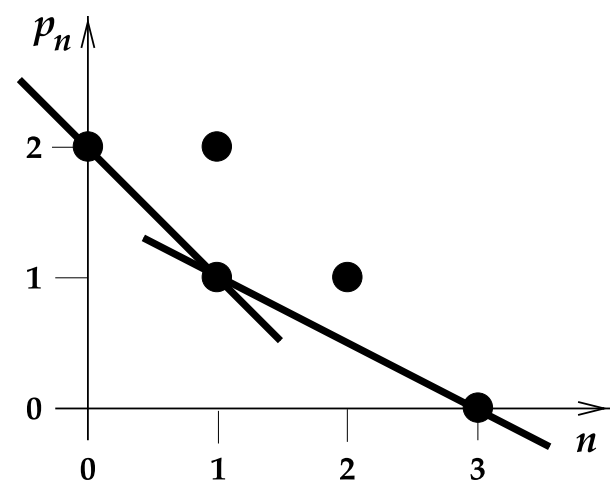

FIGURE 3. Kruskal diagram for the neutral gas, showing that the eigenmodes are a thermal-diffusion mode (1-0 balance) and two sound waves (3-1 balance).

$d$-dimensional ideal gas for $d=3$ whereas the correct coefficient of 3 corresponds to $d=1$. The Braginskii equations incorrectly assume that strong collisions have isotropized the wave motion. ${ }^{59}$ Furthermore, although the Braginskii equations predict (an incorrect formula for) dissipative collisional damping (not written in (F12)), collisionless Landau damping is absent. ${ }^{60}$

Of course, the plasma oscillations are a consequence of the long-ranged nature of the Coulomb force. It is instructive to consider the limit of a neutral gas by letting $\omega_{\mathrm{p}}^{2} \rightarrow 0$. Then the dominant terms for $\lambda^{0}$ and $\lambda^{1}$ move up to $\infty$; the corresponding Kruskal diagram is shown in figure 3 . The $1-0$ balance now leads to

$$
\lambda=-\frac{3}{5} k^{2} \chi_{\mathrm{v}}=-k^{2} \kappa / c_{\mathrm{p}},
$$

where $c_{\mathrm{p}} \doteq 5 / 2$ is the specific heat at constant pressure for a three-dimensional ideal gas. This is again a thermal-diffusion mode, but with a thermodynamics that differs from that of the OCP. ${ }^{61}$ The $3-1$ balance leads to two sound waves:

$$
\lambda= \pm k c_{\mathrm{s}},
$$

${ }^{59}$ This observation is not made in the otherwise excellent massive tome on statistical mechanics by Balescu (1975), who obtains in his $\$ 12.7$ a result that reduces to the incorrect (F 12) for weakly coupled plasma.

${ }^{60}$ Hammett \& Perkins (1990) discuss a useful method for incorporating collisionless effects into fluid equations.

${ }^{61}$ The presences of $c_{\mathrm{V}}$ in the plasma formula $(\mathrm{F} 11)$ and $c_{\mathrm{p}}$ in the neutral-fluid result (F 13) are easy to understand on physical grounds. In the neutral fluid, the dominant balance in the thermal-diffusion mode is $\Delta n / n \approx-\Delta T / T$ (i.e. $\Delta p \approx 0$ ); the diffusion of heat occurs at constant pressure. In the plasma, the dominant balance is instead between the electric force and the pressure force. Because of the long-ranged nature of the Coulomb interaction, only a small amount of density fluctuations is required in order to provide a substantial electric field at long wavelengths; as a consequence, in the plasma thermal-diffusion mode $\Delta n / n \ll \Delta T / T$. The density in a volume element of volume $V$ containing $N$ particles is $n=N / V$. Since no particles are exchanged in a thermal-diffusion process, one has $\Delta n / n=-\Delta V / V$. Since to lowest order $\Delta n / n=0$, the process occurs at constant volume. The dominant balances can be seen in a way that is more physical than the dispersion relation (F 10) by eliminating $\Delta n$ and $\Delta T$ in $(\mathrm{F} 9 b)$ :

$$
\lambda \Delta u=\underbrace{\frac{-\mathrm{i} \omega_{\mathrm{p}}^{2}}{k}\left(\frac{-\mathrm{i} k \Delta u}{\lambda}\right)}_{\text {(a) }}-\mathrm{i} v_{\mathrm{t}}^{2} k(\underbrace{\frac{-\mathrm{i} k \Delta u}{\lambda}}_{\text {(b) }}-\underbrace{\frac{(2 / 3) \mathrm{i} k \Delta u}{\lambda+\chi_{\mathrm{v}} k^{2}}}_{\text {(c) }})-\left(\frac{4}{3} \mu+\zeta\right) k^{2} \Delta u .
$$

The neutral-fluid balance is between terms (b) and (c), while the plasma balance is between terms (a) and (c). (In the latter, the $O\left(k^{4}\right)$ correction to $\lambda=-\chi_{\mathrm{v}} k^{2}$ is required in order to balance the leading-order $k^{-2}$ dependence of each term.) 
where

$$
c_{\mathrm{s}}^{2} \doteq \frac{5}{3} v_{\mathrm{t}}^{2}
$$

is the ideal-gas limit of the well-known result

$$
c_{\mathrm{s}}^{2}=\left(\frac{c_{\mathrm{p}}}{c_{\mathrm{v}}}\right) \frac{1}{m}\left(\frac{\partial p}{\partial n}\right)_{T}=\frac{1}{m}\left(\frac{\partial p}{\partial n}\right)_{s},
$$

where $s$ denotes entropy density. That long-range forces lead to profound modifications in linear-response theory is well known; good discussions are given by Martin (1967, 1968).

\section{F.2. Eigenmodes of a two-species magnetised plasma for $\boldsymbol{B}=\boldsymbol{0}$}

Next I address the generalization of the previous results to a two-species, unmagnetised plasma. The linearized continuity equations are unchanged in form. To the linearized momentum equations must be added the perturbed momentum transfer:

$$
\Delta \boldsymbol{R}_{e}=-(n m)_{e} \tau_{e}^{-1} \alpha\left(\Delta \boldsymbol{u}_{e}-\Delta \boldsymbol{u}_{i}\right)-\beta n_{e} \mathrm{i} \boldsymbol{k} \Delta T_{e}, \quad \Delta \boldsymbol{R}_{i}=-\Delta \boldsymbol{R}_{e},
$$

where $\alpha \doteq 0.51$ and $\beta \doteq 0.71$. Also, to the linearized temperature equations must be added a temperature equilibration term $\Delta Q$ :

$$
\Delta Q_{e}=-3\left(\frac{m_{e}}{m_{i}}\right) \tau_{e}^{-1}\left(\Delta T_{e}-\Delta T_{i}\right), \quad \Delta Q_{i}=-\Delta Q_{e} .
$$

Finally, the electron heat flow must be generalized to

$$
\Delta \boldsymbol{q}_{e}=-n_{e} \kappa_{e} k^{2} \mathrm{i} \boldsymbol{k} \Delta T_{e}+\beta(n T)_{e}\left(\Delta \boldsymbol{u}_{e}-\Delta \boldsymbol{u}_{i}\right) .
$$

A decomposition into decoupled longitudinal and transverse components can be made as before. The transverse dispersion relation leads to two pairs of eigenvalues, where each pair obeys

$$
\lambda^{2}+\left(v_{e}+v_{i}+\epsilon_{e}+\epsilon_{i}\right) \lambda+\epsilon_{i} v_{e}+\epsilon_{e} v_{i}+\epsilon_{e} \epsilon_{i},
$$

where $v_{e} \doteq \alpha \tau_{e}^{-1}, v_{i} \doteq\left(m_{e} / m_{i}\right) v_{e}$, and $\epsilon_{s} \doteq \mu_{s} k^{2}$. The approximate solutions are a momentum-decay mode (2-1 balance),

$$
\lambda_{-}=-\left(v_{e}+v_{i}\right)+O(\epsilon),
$$

and a momentum-diffusion mode (1-0 balance),

$$
\lambda_{+}=-\left(\frac{\epsilon_{i} \nu_{e}+\epsilon_{e} \nu_{i}}{v_{e}+v_{i}}\right)+O\left(\epsilon^{2}\right) .
$$

If one assumes $T_{e}=T_{i}=T$ and writes $\mu_{s}=C_{s} T /\left(m_{s} v_{s}\right)$, where $C_{s}$ is a constant, this eigenvalue reduces to

$$
\lambda_{+}=-T\left(\frac{C_{i}}{m_{e}}+\frac{C_{e}}{m_{i}}\right)\left(\frac{1}{v_{e}+v_{i}}\right) k^{2},
$$

describing diffusion with a hybrid viscosity based on (essentially) the reduced mass and the total collision frequency.

Remaining are six longitudinal eigenmodes, for which I merely quote the lowestorder results: 
(i) two plasma oscillations (6-4 balance): $\lambda= \pm \mathrm{i} \omega_{\mathrm{p}}$, where $\omega_{\mathrm{p}}^{2} \doteq \sum_{s} \omega_{\mathrm{p} s}^{2}$ and $\omega_{\mathrm{p} s} \doteq$ $\left[4 \pi\left(n q^{2} / m\right)_{s}\right]^{1 / 2}$

(ii) two ion sound waves (4-2 balance): $\lambda= \pm \mathrm{i} k c_{\mathrm{s}}$, where $c_{\mathrm{s}} \doteq\left(Z T_{e} / m_{i}\right)^{1 / 2}$;

(iii) a temperature-equilibration mode (2-1 balance): $\lambda=-2 \gamma$, where $\gamma \doteq 2 \nu_{i e}$;

(iv) a thermal-diffusion mode (1-0 balance): $\lambda=-(1 / 2)\left(\Sigma_{T e}+\Sigma_{T i}\right)$, where $\Sigma_{T s} \doteq$ $2 k^{2} \kappa_{s} / 3=\kappa_{s} / c_{\mathrm{v}}$.

\section{F.3. Eigenmodes of a two-species magnetised plasma for $v /\left|\omega_{\mathrm{c}}\right| \ll 1$}

Whereas for $\boldsymbol{B}=\mathbf{0}$ decomposition of $\boldsymbol{u}$ into longitudinal and transverse components is natural ( $\boldsymbol{k}$ being the only vector in the problem other than $\boldsymbol{u}$ ), for $\boldsymbol{B} \neq \mathbf{0}$ a more useful and physically meaningful decomposition is into compressional and vortical components:

$$
\Omega_{\|} \doteq \mathrm{i} k_{\|} u_{\|}, \quad \Omega_{\perp} \doteq \mathrm{i} \boldsymbol{k}_{\perp} \cdot \boldsymbol{u}_{\perp}, \quad \Omega_{\mathrm{x}} \doteq \mathrm{i}\left(\boldsymbol{k}_{\perp} \times \boldsymbol{u}\right) \cdot \widehat{\boldsymbol{b}}
$$

Expressing the algebra in this way helps one to make contact with predictions of the gyrokinetic formalism, in which vorticity plays a prominent role. In gyrokinetics, ${ }^{62}$ the perpendicular dielectric constant $\mathcal{D}_{\perp} \doteq \omega_{\mathrm{p} i}^{2} / \omega_{\mathrm{c} i}^{2}$ is assumed to be large. ${ }^{63}$ To introduce $\mathcal{D}_{\perp}$ naturally, it is convenient to normalize frequencies to $\omega_{\mathrm{c} i}$ and wavenumbers to $\rho_{\mathrm{s}} \doteq c_{\mathrm{s}} / \omega_{\mathrm{c} i}$.

I shall assume that $\epsilon \doteq v_{e} /\left|\omega_{c e}\right|$ is small. The normalized collision frequencies are then $\bar{v}_{e} \doteq v_{e} / \omega_{\mathrm{c} i}=\left(\left|\omega_{\mathrm{c} e}\right| / \omega_{\mathrm{c} i}\right)\left(v_{e} /\left|\omega_{\mathrm{c} e}\right|\right)=\epsilon / \mu, \bar{v}_{i} \doteq v_{i} / \omega_{\mathrm{c} i}=\mu \bar{v}_{e}=\epsilon$. For an optimal ordering, I shall take $\epsilon=\mu \bar{\epsilon}$, where $\bar{\epsilon}=O(1)$ with respect to $\mu$. This makes $\bar{v}_{e}=O(1)$ in the mass-ratio ordering; later, one can do a subsidiary ordering with respect to $\bar{\epsilon}$.

When considering various limiting cases in the small parameters $\mu, \epsilon$, and $\delta \doteq$ $\bar{k}^{2} \doteq k^{2} \rho_{\mathrm{s}}^{2}$ (where $k$ refers to either $k_{\|}$or $k_{\perp}$ ), it is important to keep in mind that the order of limits may matter. For example, any effect involving $k^{2}$ is small relative to the interspecies collisional relaxation rates as $k \rightarrow 0$. However, to ensure proper cancellations relating to momentum conservation when evaluating $\operatorname{det}(\boldsymbol{K})$, one must first express all collision rates in terms of a common collision frequency. Since I am treating $\bar{v}_{e}$ as $O(1)$, it is appropriate to replace $\bar{\nu}_{i} \rightarrow \mu \bar{v}_{e}$. Although the mass ratio $\mu$ is very small and the full determinant contains terms of various orders in $\mu$, most of which can be neglected, the ordered limit $\lim _{\delta \rightarrow 0} \lim _{\mu \rightarrow 0}$ will produce unusual answers since it assumes that the $k^{2}$ effects are large compared to the interspecies relaxation rates. The proper hydrodynamic limit is $\lim _{\mu \rightarrow 0} \lim _{\delta \rightarrow 0}$.

Regarding the hydrodynamic limit, note that classical transport assumes the ordering $k^{2} \lambda_{\mathrm{mfp}}^{2} \sim\left(\lambda_{\mathrm{mfp}} / L\right)^{2} \ll 1$, where $\lambda_{\mathrm{mfp}} \doteq v_{\mathrm{t}} / v$ and $L$ is a characteristic gradient scale length. In the large- $\mathcal{D}_{\perp}$ limit, the natural dimensionless wavenumber that appears is $k \rho_{\mathrm{s}}$. Since $\lambda_{\mathrm{mfp}} \gg \rho_{\mathrm{s}}$ in a hot plasma and $k_{\perp} \lambda_{\mathrm{mfp}}=\left(\lambda_{\mathrm{mfp}} / \rho_{\mathrm{s}}\right)\left(k_{\perp} \rho_{\mathrm{s}}\right) \gg k_{\perp} \rho_{\mathrm{s}}$, the requirement $k_{\perp} \lambda_{\text {mfp }} \ll 1$ does not inevitably require $k_{\perp} \rho_{\mathrm{s}} \ll 1$. However, that limit is implied by the assumption that $k_{\perp} L=O(1)$ provided that $\rho_{\mathrm{s}} / L \ll 1$.

In general, I construct from the linearized Braginskii equations a $10 \times 10$ matrix that acts on the column vector $\left(\Delta n_{e} / n_{e}, \Delta n_{i} / n_{i}, \Omega_{\| e} / \omega_{\mathrm{c} i}, \Omega_{\| i} / \omega_{\mathrm{c} i}, \Omega_{\perp e} / \omega_{\mathrm{c} i}, \Omega_{\perp i} / \omega_{\mathrm{c} i}\right.$, $\left.\Omega_{\times e} / \omega_{\mathrm{c} i}, \Omega_{\times i} / \omega_{\mathrm{c} i}, \Delta T_{e} / T_{e}, \Delta T_{i} / T_{i}\right)^{\mathrm{T}}$. A complete description of the eigenvalues and eigenvectors of that matrix for all possible limits in the multidimensional space of small parameters is beyond the scope of this paper. Below I shall merely consider a few illustrative special cases.

\footnotetext{
${ }^{62}$ For an introductory review of gyrokinetics with many references, see Krommes (2012).

${ }^{63}$ For some discussion of various regimes, see Krommes, Lee \& Oberman (1986, § II).
} 
F.3.1. Magnetized eigenvalues in the limit of zero dissipation

When all of the dissipation parameters as well as the gyroviscous stresses are set to zero (as discussed above, this is not the hydrodynamic limit because I hold $k_{\|}$and $k_{\perp}$ finite), and with $\bar{\lambda} \doteq \lambda / \omega_{\mathrm{ci}}$, one finds

$$
\boldsymbol{K}=\left(\begin{array}{cccccccccc}
-\bar{\lambda} & 0 & -1 & 0 & -1 & 0 & 0 & 0 & 0 & 0 \\
0 & -\bar{\lambda} & 0 & -1 & 0 & -1 & 0 & 0 & 0 & 0 \\
\widehat{E}_{\|}+\bar{k}_{\|}^{2} & -\widehat{E}_{\|} & -\mu \bar{\lambda} & 0 & 0 & 0 & 0 & 0 & \bar{k}_{\|}^{2} & 0 \\
-\widehat{E}_{\|} & \widehat{E}_{\|}+\tau \bar{k}_{\|}^{2} & 0 & -\bar{\lambda} & 0 & 0 & 0 & 0 & 0 & \tau \bar{k}_{\|}^{2} \\
\widehat{E}_{\perp}+\bar{k}_{\perp}^{2} & -\widehat{E}_{\perp} & 0 & 0 & -\mu \bar{\lambda} & 0 & -1 & 0 & \bar{k}_{\perp}^{2} & 0 \\
-\widehat{E}_{\perp} & \widehat{E}_{\perp}+\tau \bar{k}_{\perp}^{2} & 0 & 0 & -0 & -\bar{\lambda} & 0 & 1 & 0 & \tau \bar{k}_{\perp}^{2} \\
0 & 0 & 0 & 0 & 1 & 0 & -\mu \bar{\lambda} & 0 & 0 & 0 \\
0 & 0 & 0 & 0 & 0 & -1 & 0 & -\bar{\lambda} & 0 & 0 \\
0 & 0 & -2 / 3 & 0 & -2 / 3 & 0 & 0 & 0 & -\bar{\lambda} & 0 \\
0 & 0 & 0 & -2 / 3 & 0 & -2 / 3 & 0 & 0 & 0 & -\bar{\lambda}
\end{array}\right),
$$

where $\tau \doteq T_{i} / T_{e}=1$ (the perturbations are around an absolute equilibrium with a common temperature), $\widehat{E}_{\|} \doteq \mathcal{D}_{\perp}\left(k_{\|}^{2} / k^{2}\right)$, and $\widehat{E}_{\perp} \doteq \mathcal{D}_{\perp}\left(k_{\perp}^{2} / k^{2}\right)$. For small $\mu$, large $\mathcal{D}_{\perp}$, and small $k_{\perp}^{2}$, the characteristic polynomial is dominantly

$$
(10 / 3)\left[\mathcal{D}_{\perp}\left(k_{\perp}^{2} / k^{2}\right)\right] \bar{k}_{\|}^{2} \bar{\lambda}^{2}+\mathcal{D}_{\perp}\left(k_{\|}^{2} / k^{2}\right) \bar{\lambda}^{4}+\mathcal{D}_{\perp}\left(k_{\|}^{2} / k^{2}\right) \bar{\lambda}^{6}+\mu \lambda^{8}+\mu^{3} \bar{\lambda}^{10}=0 .
$$

Clearly, two eigenvalues vanish; those will be resolved when dissipation is included. For the remaining eigenvalues, first balance with respect to small $\mu$. The 10-8 balance gives $\bar{\lambda}^{2}=-\mu^{-2}$, which when the normalizations are unwrapped gives $\omega^{2}=\omega_{\mathrm{ce}}^{2}$, the low-density limit of the upper hybrid wave. The 8-6 balance gives $\bar{\lambda}^{2}=-\mathcal{D}_{\perp}\left(k_{\|}^{2} / k^{2}\right)$. These modes are the strongly magnetised limit of the plasma oscillations; in gyrokinetics, they are known as the $\omega_{\mathrm{H}}$ modes. For the remaining balances, which involve $\mu^{0}$, use a subsidiary ordering with respect to small $\bar{k}_{\|}$. The 6-4 balance gives $\bar{\lambda}^{2}=-1$, the ion cyclotron wave. The $4-2$ balance gives $\bar{\lambda}^{2}=-(10 / 3) \bar{k}_{\|}^{2}$. These are the ion sound waves, but with a thermodynamic coefficient that differs from the value of 1 that follows from collisionless kinetic theory. The issue here is the same one that was raised in the discussion of the incorrect thermal correction to the unmagnetised plasma oscillations (F 12); the Braginskii equations are correct only for the collisional limit $\omega \ll v$.

\section{F.3.2. Magnetized eigenvalues for purely perpendicular propagation with no dissipation}

For purely perpendicular propagation, the characteristic polynomial derived from (F25) changes to $\mathcal{D}_{\perp} \mu \bar{\lambda}^{6}+\mu \bar{\lambda}^{8}+\mu^{3} \bar{\lambda}^{10}=0$. The sound waves have disappeared; four vanishing eigenvalues will be resolved by dissipation. Furthermore, the 8-6 balance is now changed to $\bar{\lambda}^{2}=-\mathcal{D}_{\perp}$ (the order of the limits $\mu \rightarrow 0$ and $\bar{k}_{\|} \rightarrow 0$ matters), or in dimensional variables $\omega^{2}=\omega_{\mathrm{p} i}^{2}$; this is the low-density limit of the lower-hybrid waves. 
F.3.3. Magnetized eigenvalues for purely perpendicular propagation with dissipation With all terms included, one finds $\boldsymbol{K}=\left(\boldsymbol{K}_{10 \times 4}^{(1 \ldots 4)}, \boldsymbol{K}_{10 \times 2}^{(5,6)}, \boldsymbol{K}_{10 \times 4}^{(7 \ldots 10)}\right)$, where

$$
\begin{aligned}
& \boldsymbol{K}^{(1 \ldots 4)} \doteq \\
& {\left[\begin{array}{cccc}
-\bar{\lambda} & 0 & -1 & 0 \\
0 & -\bar{\lambda} & 0 & -1 \\
\widehat{E}_{\|}+\bar{k}_{\|}^{2} & -\widehat{E}_{\|} & -\mu\left(\bar{\lambda}+\alpha \bar{\nu}_{e}+\frac{4}{3} \bar{\Sigma}_{0 \| e}^{u}+\bar{\Sigma}_{2 \perp e}^{u}\right) & \mu \alpha \bar{\nu}_{e} \\
-\widehat{E}_{\|} & \widehat{E}_{\|}+\tau \bar{k}_{\|}^{2} & \alpha \bar{\nu}_{i} & -\left(\bar{\lambda}+\alpha \bar{\nu}_{i}+\frac{4}{3} \bar{\Sigma}_{0 \| i}^{u}+\bar{\Sigma}_{2 \perp i}\right) \\
\widehat{E}_{\perp}+\bar{k}_{\perp}^{2} & -\widehat{E}_{\perp} & \mu\left(\frac{2}{3} \bar{\Sigma}_{0 \perp e}^{u}-\bar{\Sigma}_{2 \perp e}^{u}\right) & 0 \\
-\widehat{E}_{\perp} & \widehat{E}_{\perp}+\tau \bar{k}_{\perp}^{2} & 0 & \frac{2}{3} \bar{\Sigma}_{0 \perp i}^{u}-\bar{\Sigma}_{2 \perp i}^{u} \\
0 & 0 & 0 & 0 \\
0 & 0 & 0 & 0 \\
0 & 0 & -\frac{2}{3}(1+\beta) & \frac{2}{3} \beta \\
0 & 0 & 0 & -\frac{2}{3}
\end{array}\right]}
\end{aligned}
$$

$$
\begin{aligned}
& \boldsymbol{K}^{(5,6)} \doteq \\
& {\left[\begin{array}{cc}
-1 & 0 \\
0 & -1 \\
\mu\left(\frac{2}{3} \bar{\Sigma}_{0 \| e}^{u}-\bar{\Sigma}_{2 \| e}^{u}\right) & 0 \\
0 & \frac{2}{3} \bar{\Sigma}_{0 \| i}^{u}-\bar{\Sigma}_{2 \| i}^{u} \\
-\mu\left(\bar{\lambda}+\bar{v}_{e}+\frac{1}{3} \bar{\Sigma}_{0 \perp e}^{u}+\bar{\Sigma}_{1 \perp e}^{u}+\bar{\Sigma}_{2 \| e}^{u}\right) & \left.\mu \bar{v}_{e} \bar{\Sigma}_{1 \perp i}^{u}+\bar{\Sigma}_{2 \| i}^{u}\right) \\
\bar{v}_{i} & -\left(\bar{\lambda}+\bar{v}_{i}+\frac{1}{3} \bar{\Sigma}_{0 \perp i}^{u}+\bar{\nu}_{1}\right. \\
1 & 0 \\
0 & 0 \\
-\frac{2}{3} & -\frac{2}{3}
\end{array}\right],} \\
& \boldsymbol{K}^{(7 \ldots 10)} \doteq\left[\begin{array}{cccc}
0 & 0 & 0 & 0 \\
0 & 0 & 0 & 0 \\
0 & 0 & (1+\beta) \bar{k}_{\|}^{2} & 0 \\
0 & 0 & -\beta \bar{k}_{\|}^{2} & \tau \bar{k}_{\|}^{2} \\
-1 & 0 & \bar{k}_{\perp}^{2} & 0 \\
0 & 1 & 0 & \tau \bar{k}_{\perp}^{2} \\
-\mu\left(\lambda+\bar{v}_{e}\right) & \mu \bar{\nu}_{e} & \frac{3}{2} \bar{k}_{\perp}^{2} \mu \bar{\nu}_{e} & 0 \\
\bar{v}_{i} & -\left(\lambda+\bar{v}_{i}\right) & 0 & -\frac{3}{2} \bar{k}_{\perp}^{2} \mu \bar{\nu}_{e} \\
\mu \bar{\nu}_{e} & -\mu \bar{\nu}_{e} & -\left(\bar{\lambda}+\bar{\gamma}+\bar{\Sigma}_{e}^{\mathrm{T}}\right) & \bar{\gamma} \\
0 & 0 & \bar{\gamma} & -\left(\bar{\lambda}+\bar{\gamma}+\bar{\Sigma}_{i}^{\mathrm{T}}\right)
\end{array}\right] .
\end{aligned}
$$

Here $v_{e} \equiv v_{e i}=\tau_{e}^{-1}$ and $v_{i} \equiv v_{i e}=\mu v_{e}$; overlines denote normalizations with respect to $\omega_{\mathrm{c} i} ; \bar{\Sigma}^{u}$ denotes quantities related to the stress tensor, with the numerical subscripts following Braginskii's notation (appendix E) and $\|$ or $\perp$ denoting $k_{\|}^{2}$ or $k_{\perp}^{2}$, e.g. $\bar{\Sigma}_{2 \perp e}^{u} \doteq k_{\perp}^{2} \mu_{2 e} / \omega_{\mathrm{c} i}$ (distinguish the mass ratio $\mu$, which is unsubscripted, from the classical viscosities $\mu_{p s}$, which are subscripted); $\bar{\Sigma}^{\mathrm{T}}$ denotes the full thermal-diffusion coefficient (e.g. $\left.\bar{\Sigma}_{e}^{\mathrm{T}} \doteq(2 / 3)\left(k_{\|}^{2} \kappa_{\| e}+k_{\perp}^{2} \kappa_{\perp e}\right) / \omega_{\mathrm{c} i}\right)$; and $\bar{\gamma} \doteq 2 \bar{v}_{i}$. 
The general dispersion relation for arbitrary $k_{\|} / k_{\perp}$ is complicated, even in the hydrodynamic limit, and will not be discussed here. The case of purely perpendicular propagation, however, is analytically tractable. With $k_{\|}=0$, small $\mu$, and large $D_{\perp}$, one finds that the characteristic polynomial is dominantly

$$
\begin{aligned}
& 2 \delta^{3} \alpha \mathcal{D}_{\perp} \epsilon^{3} \bar{\Sigma}_{2 \perp i}^{u}\left(\bar{\Sigma}_{e}^{\mathrm{T}}+\bar{\Sigma}_{i}^{\mathrm{T}}\right) \bar{k}_{\perp}^{2}(1+\tau) \bar{\lambda}+2 \delta^{2} \alpha \mathcal{D}_{\perp} \epsilon^{2} \bar{\Sigma}_{2 \perp i}^{u}\left(\bar{\Sigma}_{e}^{\mathrm{T}}+\bar{\Sigma}_{i}^{\mathrm{T}}\right) \bar{\lambda}^{2} \\
& \quad+2 \delta \alpha \mathcal{D}_{\perp} \epsilon^{2}\left(2 \bar{\Sigma}_{2 \perp i}^{u}+\bar{\Sigma}_{e}^{\mathrm{T}}+\bar{\Sigma}_{i}^{\mathrm{T}}\right) \bar{\lambda}^{3}+4 \alpha \mathcal{D}_{\perp} \epsilon^{2} \bar{\lambda}^{4} \\
& \quad+\alpha \mathcal{D}_{\perp} \epsilon \bar{\lambda}^{5}+\mathcal{D}_{\perp} \mu \bar{\lambda}^{6}+\alpha \epsilon \bar{\lambda}^{7}+\mu \bar{\lambda}^{8}+(2+\alpha) \mu^{2} \epsilon \bar{\lambda}^{9}+\mu^{3} \bar{\lambda}^{10}
\end{aligned}
$$

where I have inserted the hydrodynamic ordering parameter $\delta$ to explicitly remind one of the order of the transport terms ${ }^{64}$ in $\bar{k}_{\perp}^{2}$. One eigenvalue vanishes; that is resolved by viscous dissipation for small but non-zero $k_{\|}$.

As stated above, the proper order of limits is $\lim _{\mu \rightarrow 0} \lim _{\delta \rightarrow 0}$. With respect to $\delta$, the dominant balances are to set to zero either the first two lines of (F 27d) (balances A) or the last term of the second line plus the last line (balances B). For the A balances, all of the terms fall on a line in a $\delta$-ordered Kruskal diagram, so there is no asymptotic simplification for small $\delta$. After one factors out $2 \alpha \mathcal{D}_{\perp} \epsilon^{2}$ and sets $\delta=1$, the A balances become

$$
\epsilon \bar{\Sigma}_{2 \perp i}^{u}\left(\bar{\Sigma}_{e}^{\mathrm{T}}+\bar{\Sigma}_{i}^{\mathrm{T}}\right) \bar{k}_{\perp}^{2}(1+\tau) \bar{\lambda}+\bar{\Sigma}_{2 \perp i}^{u}\left(\bar{\Sigma}_{e}^{\mathrm{T}}+\bar{\Sigma}_{i}^{\mathrm{T}}\right) \bar{\lambda}^{2}+\left(2 \bar{\Sigma}_{2 \perp i}^{u}+\bar{\Sigma}_{e}^{\mathrm{T}}+\bar{\Sigma}_{i}^{\mathrm{T}}\right) \bar{\lambda}^{3}+2 \bar{\lambda}^{4}=0 .
$$

With respect to $\epsilon$, the dominant balances are $2-1$ and $4-3-2$. The $2-1$ balance is

$$
\bar{\lambda}=-(1+\tau) \bar{k}_{\perp}^{2} \epsilon \quad \text { or } \lambda=-(1+\tau) k_{\perp}^{2} D_{\perp},
$$

where $D_{\perp} \doteq \rho_{i}^{2} v_{i}=\rho_{e}^{2} v_{e}$; this is the ambipolar cross-field density-diffusion mode. The 4-3-2 balance factors into

$$
\left(\bar{\lambda}+\bar{\Sigma}_{2 \perp i}^{u}\right)\left[\bar{\lambda}+\frac{1}{2}\left(\bar{\Sigma}_{e}^{\mathrm{T}}+\bar{\Sigma}_{i}^{\mathrm{T}}\right)\right]=0,
$$

yielding a momentum-diffusion mode and a thermal-diffusion mode.

The B balances do not involve $\delta$, so one is free to consider balances with respect to $\mu$. Upon factoring out $\bar{\lambda}^{4}$ and replacing $\epsilon=\mu \bar{\epsilon}$, one has

$$
4 \alpha \mathcal{D}_{\perp} \mu^{2} \bar{\epsilon}^{2}+\alpha \mathcal{D}_{\perp} \mu \bar{\epsilon} \bar{\lambda}+\mathcal{D}_{\perp} \mu \bar{\lambda}^{2}+\alpha \mu \bar{\epsilon} \bar{\lambda}^{3}+\mu \bar{\lambda}^{4}+(2+\alpha) \mu^{3} \bar{\epsilon} \bar{\lambda}^{5}+\mu^{3} \bar{\lambda}^{6}=0,
$$

which contains the balances $1-0,4-3-2-1$, and $6-4$. The $1-0$ balance yields

$$
\bar{\lambda}=-4 \mu \bar{\epsilon}=-4 \epsilon \text { or } \lambda=-4 v_{i} ;
$$

this is an interspecies heat-relaxation mode. The 4-3-2-1 balance can be further ordered with respect to large $\mathcal{D}_{\perp}$, yielding (i) the 2-1 balance

$$
\bar{\lambda}=-\alpha \bar{\epsilon} \quad \text { or } \lambda=-\alpha v_{e},
$$

which is an interspecies momentum-relaxation mode; and (ii) the 4-2 balance

$$
\bar{\lambda}^{2}=-\mathcal{D}_{\perp} \quad \text { or } \lambda^{2}=-\omega_{\mathrm{p} i}^{2},
$$

${ }^{64}$ Since $\delta$ has been included explicitly, one must treat $\bar{k}_{\perp}^{2}$ as order unity in (F27d). 
the lower-hybrid waves. Finally, the $6-4$ balance yields

$$
\bar{\lambda}^{2}=-\mu^{-2} \text { or } \lambda^{2}=-\omega_{\mathrm{ce}}^{2},
$$

the upper-hybrid waves.

For the particular orderings I chose, the eigenmodes obtained for perpendicular propagation are physically reasonable; clearly, though, other orderings will lead to different results. In the general case, numerical work is essential. But the analytical exploration of the linearized Braginskii equations provides a good example of several important lessons from asymptotic analysis, namely that the order of limits can matter and that dominant balances in polynomial equations can be usefully analysed in terms of Kruskal diagrams.

Finally, I emphasize that not all of the Braginskii eigenmodes are hydrodynamic (i.e. some modes such as the Langmuir oscillations do not vanish with $k$ ). In a projection-operator formalism, such non-hydrodynamic modes must be treated with care. See appendix G, especially §G.2, for more discussion.

\section{Appendix G. Projection operators: caveats and further examples}

As shown in the main text, an appropriate choice of projection operator leads to a systematic derivation of multispecies transport theory, at least to first order in the gradients. (See Part 2 for a discussion of second-order effects.) However, although the projection method is quite powerful, it can be misused, as I shall show in $\S \S$ G.1-G.3 with several simple examples. Finally, in $\S$ G.4 I shall discuss the Brownian test particle in terms of two possible projections. In this case, there is no misuse of the formalism; both projections are viable. Understanding why that is so, in the face of the caveats discussed in the next three subsections, should lead one to a deeper appreciation for the overall content and consistency of the formalism.

\section{G.1. Projection-operator methods and non-locality}

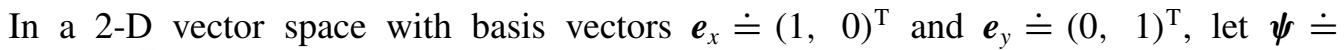
$\left(\psi_{x}, \psi_{y}\right)^{\mathrm{T}}$ obey

$$
\partial_{t} \boldsymbol{\psi}+\mathrm{i} \boldsymbol{L} \cdot \boldsymbol{\psi}=0
$$

where

$$
L=\left(\begin{array}{cc}
0 & \mathrm{i} \\
-\mathrm{i} & 0
\end{array}\right)
$$

(Note that the eigenvalues of $L$ are \pm 1 , indicating oscillation.) The resulting dynamical system is

$$
\dot{\psi}_{x}=\psi_{y}, \quad \dot{\psi}_{y}=-\psi_{x} .
$$

These can be combined into the wave equation

$$
\ddot{\psi}_{x}+\psi_{x}=0
$$

so the variables oscillate sinusoidally with unit frequency. 
Let us try to recover $(\mathrm{G} 4)$ by projecting $(\mathrm{G} 1)$ into the $x$ direction. That can be accomplished by introducing the projection operator

$$
\boldsymbol{P} \doteq \boldsymbol{e}_{x} \otimes \boldsymbol{e}_{x}^{\mathrm{T}}=\left(\begin{array}{ll}
1 & 0 \\
0 & 0
\end{array}\right) .
$$

The standard equations apply:

$$
\begin{gathered}
\partial_{t} \boldsymbol{P} \cdot \boldsymbol{\psi}+(\boldsymbol{P} \cdot \mathrm{i} \boldsymbol{L} \cdot \boldsymbol{P}) \boldsymbol{P} \cdot \boldsymbol{\psi}=-(\boldsymbol{P} \cdot \mathrm{i} \boldsymbol{L} \cdot \boldsymbol{Q}) \cdot \boldsymbol{Q} \cdot \boldsymbol{\psi}, \\
\partial_{t} \boldsymbol{Q} \cdot \boldsymbol{\psi}+(\boldsymbol{Q} \cdot \mathrm{i} \boldsymbol{L} \cdot \boldsymbol{Q}) \cdot \boldsymbol{Q} \cdot \boldsymbol{\psi}=-(\boldsymbol{Q} \cdot \mathrm{i} \boldsymbol{L} \cdot \boldsymbol{P}) \cdot \boldsymbol{P} \cdot \boldsymbol{\psi}
\end{gathered}
$$

Simple calculation shows that the frequency matrix $\boldsymbol{P} \cdot \boldsymbol{L} \cdot \boldsymbol{P}$ vanishes identically for the present problem, so in spite of its name it is unrelated to the natural oscillation. Furthermore, although the standard procedure is to eliminate $\boldsymbol{Q} \cdot \boldsymbol{\psi}$ by introducing Green's function $\boldsymbol{G}_{\boldsymbol{Q}}(\tau) \doteq H(\tau) \exp (-\boldsymbol{Q} \cdot \mathrm{i} \boldsymbol{L} \cdot \boldsymbol{Q} \tau)$, so

$$
\boldsymbol{Q} \cdot \boldsymbol{\psi}(t)=-\int_{0}^{t} \mathrm{~d} \tau \boldsymbol{G}_{Q}(\tau) \cdot \boldsymbol{Q} \cdot \mathrm{i} \boldsymbol{L} \cdot \boldsymbol{P} \cdot \boldsymbol{\psi}(t-\tau),
$$

it is easy to show that in the present problem $\boldsymbol{Q} \cdot \boldsymbol{L} \cdot \boldsymbol{Q} \equiv \boldsymbol{0}$. Thus, $\boldsymbol{G}_{\boldsymbol{Q}}(\tau)$ does not decay in time, precluding the possibility of a Markovian description. Indeed, upon noting that

$$
\boldsymbol{Q} \cdot \boldsymbol{L} \cdot \boldsymbol{P}=(\boldsymbol{I}-\boldsymbol{P}) \cdot \boldsymbol{L} \cdot \boldsymbol{P}=\boldsymbol{L} \cdot \boldsymbol{P}=\left(\begin{array}{cc}
0 & 0 \\
-\mathrm{i} & 0
\end{array}\right),
$$

one finds that

$$
\boldsymbol{Q} \cdot \boldsymbol{\psi}(t)=-\int_{0}^{t} \mathrm{~d} \bar{t}\left(\begin{array}{c}
0 \\
\psi_{x}(\bar{t})
\end{array}\right)
$$

then, with

$$
\boldsymbol{P} \cdot \boldsymbol{L} \cdot \boldsymbol{Q}=\boldsymbol{P} \cdot \boldsymbol{L} \cdot(\boldsymbol{I}-\boldsymbol{P})=\boldsymbol{P} \cdot \boldsymbol{L}=\left(\begin{array}{ll}
0 & \mathrm{i} \\
0 & 0
\end{array}\right),
$$

one finds that $(\mathrm{G} 6 a)$ becomes

$$
0=\frac{\partial}{\partial t}\left(\begin{array}{c}
\psi_{x}(t) \\
0
\end{array}\right)+\int_{0}^{t} \mathrm{~d} \bar{t}\left(\begin{array}{c}
\psi_{x}(\bar{t}) \\
0
\end{array}\right)
$$

The desired $x$ component can be extracted by dotting with $\boldsymbol{e}_{x}^{\mathrm{T}}$. Of course, the non-local integro-differential equation that results is equivalent to the second-order differential equation (G4).

This trivial example shows that it is not inevitable that a projected description must be time-local or Markovian. Note that the original vector system (G1) of coupled ordinary differential equations (ODEs) is local in time; time-history integration is introduced as a consequence of the projection. ${ }^{65}$ This is actually typical behaviour when the system supports linear waves. Very special conditions must hold in order that a projected system is Markovian. At least, what is required is that

${ }^{65}$ This phenomenon is well known in the theory of classical Brownian motion (Wang \& Uhlenbeck 1945). 
Green's function $\mathrm{G}_{\mathrm{Q}}(\tau)$ (an operator) decays sufficiently rapidly in time when acting on the orthogonal subspace (as is required for $(\mathrm{G} 6 b)$ ), a property that is related to the spectrum of QLQ. Note that QLQ will always possess a null space that is at least $p$-dimensional, where $p$ is the dimension of the projected subspace, since (QLQ) $\mathrm{P}=0$. But if it also possesses a zero or very small eigenvalue in the $\mathrm{Q}$ direction (as in the present example), some sort of non-locality must ensue. It is for this reason that for applications to fluid equations $Q$ is chosen to project into the directions orthogonal to the null space of the collision operator. ${ }^{66}$

\section{G.2. Projection operators and the dispersion relation of Langmuir oscillations}

One of the wonderful yet dangerous features of the projection-operator formalism is that one can project onto virtually anything. For the case of collisional transport, the choice of projection operator is relatively obvious. In general, however, the formalism can be confusing because the zero-frequency limit is not allowed for high-frequency modes and because of the appearance of $\mathrm{Q}$ in the relevant Green's function.

I remarked in $\S \mathrm{F} .1$ that the Braginskii equations predict an incorrect dispersion relation for the Langmuir oscillations $\omega=\Omega_{k} \approx \pm \omega_{\mathrm{p}}$ as a consequence of a violation of the Markovian approximation by those high-frequency modes. Before the Markovian approximation is made, however, a projection-operator formalism must produce a formally correct result, no matter into what space the dynamics is projected. The proper dispersion relation, including both reactive and dissipative parts, must follow from a correct evaluation of the frequency-dependent $\widehat{\Sigma}(\omega)$. I shall demonstrate this by analysing the projection into the density subspace for the well-known model problem of linearized, collisionless Vlasov response. Note that for a high-frequency mode the null eigenspace of the collision operator is no longer relevant and cannot be used to motivate a useful projection operator. Projecting into the 1-D density subspace is the simplest operation that can be done, yet it demonstrates some non-trivial manipulations.

Thus, consider ${ }^{67} \mathrm{P}=|1\rangle\langle 1|$ and project the perturbed kinetic equation (2.12), assuming a collisionless electron plasma with neutralizing ion background. The frequency matrix involves the single matrix element $\langle 1|\boldsymbol{v}| 1\rangle=\mathbf{0}$. The electric-field term vanishes under $\mathrm{P}$ because it is a perfect derivative in velocity space. Thus, the perturbed density evolves according to

$$
-\mathrm{i} \omega\left(\frac{\Delta \widehat{n}(\boldsymbol{k}, \omega)}{n}\right)+\widehat{\Sigma}(\boldsymbol{k}, \omega)\left(1+\frac{1}{k^{2} \lambda_{\mathrm{D} e}^{2}}\right)\left(\frac{\Delta \widehat{n}(\boldsymbol{k}, \omega)}{n}\right)=\frac{\Delta n(0)}{n},
$$

\footnotetext{
${ }^{66}$ Projection into the directions orthogonal to the null space of the collision operator is necessary but not sufficient. When that operator is the linearized Landau operator (or the linearized Boltzmann operator for neutral fluids), it is clear from the discussion in $\S \mathrm{B} .4$ that perturbations in the orthogonal directions decay rapidly. But the famous example of the long-time algebraic tails of correlation functions $\left(C(\tau) \sim \tau^{-d / 2}\right.$, where $d$ is the dimension of space) discovered by Alder \& Wainwright (1970) shows that those familiar operators omit essential physics. Specifically, collisional processes at the microscopic level excite long-lived hydrodynamic excitations, and the nonlinear mode coupling of those fluctuations leads to a slow component that does not lie in the null space of the standard operators. In magnetised plasmas, the phenomenon is called the generation of convective cells and was treated by Krommes \& Oberman (1976). (For a discussion of the neutral-fluid problem, see Balescu (1975, §21.5), Reichl (1998, §11.A), Zwanzig (2001, chap. 9), and the references listed in Krommes \& Oberman (1976).) One method of attack is to augment the dimensionality of the standard hydrodynamic projector $\mathrm{P}$ to include the extra slow directions, as was done by Krommes \& Oberman (1976). The resulting effect on the transport coefficients is particularly severe in two dimensions, where the transport is distinctly non-local. (An attempt at a Markovian description leads to divergent transport coefficients, $\eta \sim \int_{0}^{\infty} \mathrm{d} \tau \tau^{-1} \propto \lim _{\tau \rightarrow \infty} \ln \tau$.)

${ }^{67}$ A closely related version of this calculation is the discussion of Krommes (1975, appendix F), which treats the short-time limit of the two-time correlation function of the many-body plasma.
} 
where $^{68}$

$$
\widehat{\Sigma}(\boldsymbol{k}, \omega) \doteq \boldsymbol{k} \cdot\left\langle\boldsymbol{v} \mathrm{Q}\left|[-\mathrm{i}(\omega \mathbf{1}-\mathrm{Q} \boldsymbol{k} \cdot \boldsymbol{v} \mathrm{Q}+\mathrm{i} \epsilon \mathbf{1})]^{-1}\right| \mathrm{Q} \boldsymbol{v}\right\rangle \cdot \boldsymbol{k}
$$

The term in $\left(k \lambda_{\mathrm{D} e}\right)^{-2}$ arises from the Q projection of the $\Delta \boldsymbol{E}$ term in (2.12) upon using Poisson's equation to express $\Delta \boldsymbol{E}$ in terms of the electron charge density. In (G 13), the left-most and right-most $\mathrm{Q}$ operators may be omitted if desired because $\mathrm{Q}=\mathbf{1}-\mathrm{P}$, $\mathrm{P}|\boldsymbol{v}\rangle=\mathbf{0}$, and $\langle\boldsymbol{v}| \mathrm{P}=\mathbf{0}$; for the same reason, it is sufficient to write $\mathrm{Q} \boldsymbol{k} \cdot \boldsymbol{v} \mathrm{Q}=\mathrm{Q} \boldsymbol{k} \cdot \boldsymbol{v}$. The matrix element, a second-rank tensor, depends only on $\boldsymbol{k}$, so it must have the form

$$
\left\langle\boldsymbol{v}\left|[-\mathrm{i}(\omega \mathbf{1}-\mathrm{Q} \boldsymbol{k} \cdot \boldsymbol{v} \mathrm{Q}+\mathrm{i} \epsilon \mathbf{1})]^{-1}\right| \boldsymbol{v}\right\rangle=a(k) \widehat{\boldsymbol{k} \boldsymbol{k}}+b(k)(\boldsymbol{I}-\widehat{\boldsymbol{k} \boldsymbol{k}}) .
$$

Only the $\widehat{\boldsymbol{k}} \widehat{\boldsymbol{k}}$ term contributes to (G13), so one must evaluate

$$
\widehat{\Sigma} \doteq\langle\boldsymbol{k} \cdot \boldsymbol{v}|\left[-\mathrm{i}(\omega \mathbf{1}-\mathrm{Q} \boldsymbol{k} \cdot \boldsymbol{v}+\mathrm{i} \epsilon \mathbf{1})^{-1}|\mathrm{Q} \boldsymbol{k} \cdot \boldsymbol{v}\rangle .\right.
$$

Addition and subtraction of $\omega$ to the right-most ket leads to

$$
\widehat{\Sigma}=-\mathrm{i} \omega\left\langle\boldsymbol{k} \cdot \boldsymbol{v} \mid(\omega \mathbf{1}-\mathrm{Q} \boldsymbol{k} \cdot \boldsymbol{v}+\mathrm{i} \epsilon \mathbf{1})^{-1}\right\rangle .
$$

The term in angular brackets is similar, though not identical, to the integral that defines the electrostatic susceptibility:

$$
\chi(\boldsymbol{k}, \omega) \doteq-\left(k \lambda_{\mathrm{D} e}\right)^{-2} J(\boldsymbol{k}, \omega),
$$

where

$$
J(\boldsymbol{k}, \omega) \doteq\left\langle\frac{\boldsymbol{k} \cdot \boldsymbol{v}}{\omega-\boldsymbol{k} \cdot \boldsymbol{v}+\mathrm{i} \epsilon}\right\rangle .
$$

To simplify ( $\mathrm{G} 16)$, use $\mathrm{Q}=\mathbf{1}-\mathrm{P}$ and define the operators

$$
\mathrm{A} \doteq(\omega-\boldsymbol{k} \cdot \boldsymbol{v}) \mathbf{1}, \quad \mathrm{B} \doteq \mathrm{P} \boldsymbol{k} \cdot \boldsymbol{v}
$$

so that

$$
J=\left\langle\mathrm{BA}^{-1}\right\rangle
$$

Then

$$
\widehat{\Sigma} /(-\mathrm{i} \omega)=\left\langle\mathrm{B}(\mathrm{A}+\mathrm{B})^{-1}\right\rangle
$$

With the aid of the operator identity (4.15), one finds $\widehat{\Sigma}=-\mathrm{i} \omega I$, where

$$
I \doteq\left\langle\mathrm{B}(\mathrm{A}+\mathrm{B})^{-1}\right\rangle=\left\langle\mathrm{BA}^{-1}\right\rangle-\left\langle\mathrm{BA}^{-1} \mathrm{~B}(\mathrm{~A}+\mathrm{B})^{-1}\right\rangle .
$$

Since $\mathrm{B}=|1\rangle\langle\boldsymbol{k} \cdot \boldsymbol{v}|$, the last term factors:

$$
\left\langle\mathrm{BA}^{-1} \mathrm{~B}(\mathrm{~A}+\mathrm{B})^{-1}\right\rangle=\left\langle\mathrm{BA}^{-1}\right\rangle\left\langle\mathrm{B}(\mathrm{A}+\mathrm{B})^{-1}\right\rangle=J I .
$$

\footnotetext{
${ }^{68}$ In this discussion I shall use the notation 1 instead of 1 for the identity operator in order to avoid confusion with scalar functions of velocity.
} 
Thus, (G 22) becomes

$$
I=J-J I,
$$

the solution of which is

$$
I=J /(1+J)
$$

Upon defining the electrostatic dielectric function as

$$
\mathcal{D}(\boldsymbol{k}, \omega) \doteq 1+\chi(\boldsymbol{k}, \omega)=1-J(\boldsymbol{k}, \omega) /\left(k^{2} \lambda_{\mathrm{D} e}^{2}\right),
$$

one readily obtains the solution to (G 12) with the aid of (G25):

$$
\frac{\Delta \widehat{n}(\boldsymbol{k}, \omega)}{n}=\left(\frac{1+J(\boldsymbol{k}, \omega)}{-\mathrm{i} \omega \mathcal{D}(\boldsymbol{k}, \omega)}\right) \frac{\Delta n(0)}{n} .
$$

Since one has

$$
1+J(\boldsymbol{k}, \omega)=1+\left\langle\frac{\boldsymbol{k} \cdot \boldsymbol{v}}{\omega-\boldsymbol{k} \cdot \boldsymbol{v}+\mathrm{i} \epsilon}\right\rangle=\omega\left\langle\frac{1}{\omega-\boldsymbol{k} \cdot \boldsymbol{v}+\mathrm{i} \epsilon}\right\rangle,
$$

the final result is

$$
\frac{\Delta \widehat{n}(\boldsymbol{k}, \omega)}{n}=\frac{1}{\mathcal{D}(\boldsymbol{k}, \omega)}\left(\int \mathrm{d} \boldsymbol{v} \frac{f_{\mathrm{M}}(\boldsymbol{v})}{-\mathrm{i}(\omega-\boldsymbol{k} \cdot \boldsymbol{v}+\mathrm{i} \epsilon)}\right) \frac{\Delta \widehat{n}(\boldsymbol{k}, t=0)}{n} .
$$

This is to be compared with the exact solution of the linearized Vlasov problem:

$$
\frac{\Delta \widehat{n}(\boldsymbol{k}, \omega)}{n}=\frac{1}{\mathcal{D}(\boldsymbol{k}, \omega)}\left(\frac{\bar{n}}{n}\right) \int \mathrm{d} \boldsymbol{v} \frac{\Delta \widehat{f}(\boldsymbol{v}, \boldsymbol{k}, t=0)}{-\mathrm{i}(\omega-\boldsymbol{k} \cdot \boldsymbol{v}+\mathrm{i} \epsilon)} .
$$

As must be so, the results agree when the initial perturbation is chosen to have the form

$$
\Delta \widehat{f}(\boldsymbol{v}, \boldsymbol{k}, t=0)=\left(\frac{\Delta \widehat{n}(\boldsymbol{k}, t=0)}{\bar{n}}\right) f_{\mathrm{M}}(\boldsymbol{v})
$$

(i.e. when only a density perturbation is imposed initially). The contribution from the vertical part of $\Delta f(0)$ is contained in the propagated initial-condition term that was ignored in the elimination of $\mathrm{Q}|\Delta \chi\rangle$. That is, the system was prepared to lie in the density subspace.

Results such as (4.15) or (G25) show that one must be very careful to not confuse unmodified propagators with ones modified with the orthogonal projector Q. Although here I was working with the single-particle propagator, similar relations arise in manybody physics, where the $N$-particle Liouville propagator arises; that was the original situation discussed by Mori (1965). Some further interpretations and generalizations of the formalism are discussed in appendices D and E of Krommes (1975). 


\section{G.3. The plateau phenomenon; unmodified vs modified propagators}

According to Kubo (1957), linear-response functions can be couched as two-time correlations of time-dependent currents for which the time dependence is induced by the Liouville propagator $\exp (-\mathrm{i} \mathcal{L} t)$. However, the transport coefficients discussed in the present article involve currents defined with the modified propagator ${ }^{69} \exp (-\mathrm{iQ} \mathcal{L Q} t)$. The subtle but crucial difference relates to the distinction between 'internal' and 'external' response functions and to a behaviour known as the plateau phenomenon (Berne 1971; Mazenko 1973, and references therein). Here I provide a brief introduction.

The simplest illustration involves the familiar stochastic Langevin equation for the random momentum $\widetilde{p}$ of an unmagnetised test particle of mass $M$ :

$$
\frac{\mathrm{d} \widetilde{p}}{\mathrm{~d} t}+v \widetilde{p}=\widetilde{f}^{\mathrm{ext}}(t)
$$

where $\widetilde{f}^{\text {ext }}$ is taken to be centred Gaussian white noise with covariance ${ }^{70}$

$$
F^{\mathrm{ext}}\left(t, t^{\prime}\right) \doteq\left\langle\widetilde{f}^{\mathrm{ext}}(t) \widetilde{f}^{\mathrm{ext}}\left(t^{\prime}\right)\right\rangle=2 D_{p} \delta\left(t-t^{\prime}\right) ;
$$

the constant $D_{p}$ is the momentum-space diffusion coefficient (at short times, $v t \ll 1$, the mean-square momentum fluctuations obey $\left.\left\langle\delta p^{2}\right\rangle(t)=2 D_{p} t\right)$. It is well known and can be easily proven from (G32) that on the collisional time scale the fluctuation level saturates at the level $\left\langle\delta p^{2}\right\rangle=D_{p} / \nu$. The steady-state balance between kinetic energy and the thermal energy of a bath at temperature $T$ then leads to the Einstein relation $D_{p} / M v=T$.

The existence of that steady state leads to a peculiar but important property of the two-time correlation function $F\left(t, t^{\prime}\right)$ of the total force

$$
\widetilde{f} \doteq-v \widetilde{p}+\widetilde{f}^{\mathrm{ext}} .
$$

From

$$
\left\langle\delta p^{2}\right\rangle(t)=\int_{0}^{t} \mathrm{~d} \bar{t} \int_{0}^{t} \mathrm{~d} \bar{t}^{\prime} F\left(\bar{t}, \bar{t}^{\prime}\right),
$$

one can calculate a running 'total diffusion coefficient' according to

$$
D_{p}^{\mathrm{tot}}(t) \doteq \frac{1}{2} \frac{\mathrm{d}\left\langle\delta p^{2}\right\rangle}{\mathrm{d} t}=\int_{0}^{t} \mathrm{~d} \bar{\tau} F(\bar{\tau}) .
$$

The fact that $\mathrm{d}\left\langle\delta p^{2}\right\rangle / \mathrm{d} t \rightarrow 0$ as $t \rightarrow \infty$ implies that rigorously

$$
\int_{0}^{\infty} \mathrm{d} \bar{\tau} F(\bar{\tau})=0
$$

Since it is easy to see that $F(\tau) \rightarrow F^{\text {ext }}(\tau)$ as $\tau \rightarrow 0$, (G 37) implies that $F(\tau)$ possesses a long, negative tail whose integrated contribution exactly cancels the diffusive contribution embodied in $F^{\text {ext }}$. This can be seen explicitly by direct calculation, which shows that

$$
F(\tau)=2 D_{p}\left(\delta(\tau)-\frac{1}{2} \nu \mathrm{e}^{-v \tau}\right)
$$




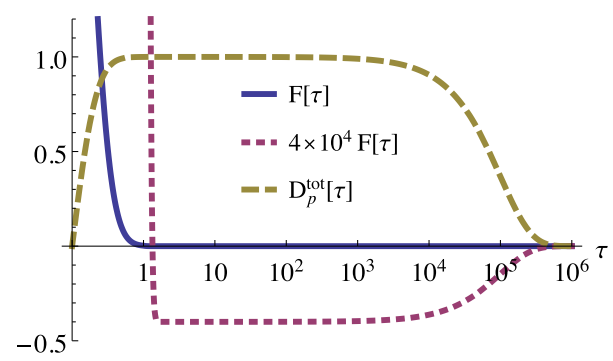

FIgURE 4. Solid curve: the function $F(\tau)$ (G38) for $D_{p}=1$; dotted curve: $4 \times 10^{4} F(\tau)$ (amplified so as to make the shape of the negative tail visible); dashed curve: the running diffusion coefficient $D_{p}^{\text {tot }}(\tau)$, showing that a plateau forms after a few autocorrelation times and that the total area under $F(\tau)$ goes to zero after a few collision times. The delta function in $(\mathrm{G} 38)$ has been opened up to be a Gaussian with standard deviation $\sigma=$ $(2 / \pi)^{1 / 2} / 3$, so that one unit in $\tau$ corresponds to 3 microscopic correlation times $\tau_{\mathrm{ac}}$. In these units, $v$ is chosen to be $10^{-5}$. The $\tau$ axis is linear for $\tau \leqslant 1$ and logarithmic for $\tau>1$.

This function and its time integral are illustrated in figure 4.

The integral $(\mathrm{G} 37)$ is the one-sided Fourier transform of $F(\tau)$ evaluated at $\omega=0$. The one-sided transform of $(\mathrm{G} 38)$ is

$$
\widehat{F}(\omega)=D_{p}-D_{p} \frac{v}{-\mathrm{i}(\omega+\mathrm{i} \nu)},
$$

or, upon dividing by $M T$ and invoking the Einstein relation,

$$
\widehat{\Phi}(\omega) \doteq \widehat{F}(\omega) /(M T)=v-v[-\mathrm{i}(\omega+\mathrm{i} v)]^{-1} v,
$$

where the last term has been written in a way that will be easy to compare with the more general formula (G 55) derived later. Another interesting form is

$$
\widehat{\Phi}^{-1}(\omega)=v^{-1}+(-\mathrm{i} \omega)^{-1} .
$$

From either (G40) or (G41), one finds

$$
\widehat{\Phi}(0)=0
$$

as a signature of the long-time tail.

More generally, the constant $v$ could be replaced by a frequency-dependent relaxation rate $\widehat{v}(\omega)$ with $\widehat{v}(0)=v$. Then the message is that whereas $\widehat{v}(\omega)$ approaches a non-zero limit as $\omega \rightarrow 0, \widehat{\Phi}(\omega)$ vanishes in that limit.

The physical difference between $\widehat{v}(\omega)$ and $\widehat{\Phi}(\omega)$ is that $\widehat{v}(\omega)$ describes an internal polarization process whereas $\widehat{\Phi}(\omega)$ describes the total response to an external perturbation. This distinction is crucial to maintain for all response processes; a

\footnotetext{
${ }^{69}$ I did not work with the Liouville operator in the present paper. That is done in Part 2, where the salient results of Part 1 are recovered from the $\Gamma$-space formalism.

${ }^{70}$ The abbreviation ext stands for external. It refers to the fact that physically $\widetilde{f}^{\text {ext }}$ arises from the random motions of the bath particles, which are external relative to the identity of a test particle.
} 
lengthy pedagogical article that includes a discussion of conductivity is by Kubo (1974).

The representations $(\mathrm{G} 40)$ or $(\mathrm{G} 41)$ might suggest that it is possible to extract the relaxation coefficient $v$ (or $D_{p}$, via the Einstein relation) from the high-frequency limit of $\widehat{\Phi}(\omega)$ :

$$
v=\lim _{\omega \rightarrow \cdot \infty} \widehat{\Phi}(\omega),
$$

where the inverted commas remind one that $\omega^{-1}>\tau_{\mathrm{ac}}$, where $\tau_{\mathrm{ac}}$ is the microscopic autocorrelation time (the physical width of the delta function in (G38)), since the Langevin model is not valid for $\tau<\tau_{\mathrm{ac}}$. In the time domain, the statement is that $D_{p}^{\text {tot }}(t) \approx D_{p}$ for $\tau_{\text {ac }}<t \ll v^{-1}$. This is a consequence of the plateau behaviour in which $D_{p}^{\text {tot }}(t)$ quickly rises to an essentially constant value, then slowly falls off as the effects of the negative tail manifest. However, this works only for frequency-independent $v$. More generally, it is better to find $v=\lim _{\omega \rightarrow 0} v(\omega)$ from the subtracted form

$$
\nu^{-1}=\lim _{\omega \rightarrow 0}\left[\widehat{\Phi}^{-1}(\omega)-(-\mathrm{i} \omega)^{-1}\right] .
$$

A generalization of this result is useful for multispecies plasmas, which contain interspecies equilibration phenomena that occur on the collisional time scale.

Now consider the general projection-operator result for the frequency-dependent hydrodynamic transport matrix: ${ }^{71}$

$$
\widehat{\boldsymbol{\Sigma}}(\omega) \doteq\left\langle\boldsymbol{A}\left|\mathcal{L} \mathrm{Q} \widehat{G}_{\mathrm{Q}}(\omega) \mathrm{Q} \mathcal{L}\right| \boldsymbol{A}\right\rangle \cdot \boldsymbol{M}^{-1}
$$

and the corresponding function $\widehat{\Phi}(\omega)$ defined with $\mathrm{G}(\omega)$ instead of $\mathrm{G}_{\mathrm{Q}}(\omega)$. These matrices are related algebraically in a natural generalization of (G40). To show this, note that

$$
\widehat{G}(\omega)=[-\mathrm{i}(\omega-\mathcal{L}+\mathrm{i} \epsilon)]^{-1},
$$

write $\mathcal{L}=\mathrm{P} \mathcal{L}+\mathrm{Q} \mathcal{L}$, and invoke the identity (4.15) with $\mathrm{A} \doteq-\mathrm{i}(\omega-\mathrm{Q} \mathcal{L})$ and $\mathrm{B} \doteq \mathrm{iP} \mathcal{L}$ to find

$$
\widehat{\boldsymbol{\Phi}}=\widehat{\boldsymbol{\Sigma}}-\left\langle\boldsymbol{A}\left|\mathcal{L} \mathrm{Q} \widehat{G}_{\mathrm{Q}} \mathrm{iP} \mathcal{L} \widehat{G} \mathrm{Q} \mathcal{L}\right| \boldsymbol{A}\right\rangle \cdot \boldsymbol{M}^{-1} .
$$

The last term, sans minus sign, is explicitly

$$
\left\langle\boldsymbol{A}\left|\mathcal{L} \mathrm{Q} \widehat{G}_{\mathrm{Q}} \mathrm{i}\right| \boldsymbol{A}^{\mathrm{T}}\right\rangle \cdot \boldsymbol{M}^{-1} \cdot\left\langle\boldsymbol{A}|\mathcal{L} \widehat{G} \mathrm{Q} \mathcal{L}| \boldsymbol{A}^{\mathrm{T}}\right\rangle \cdot \boldsymbol{M}^{-1} .
$$

In the first matrix element of (G48), write

$$
[-\mathrm{i}(\omega-\mathrm{Q} \mathcal{L})]^{-1}=(-\mathrm{i} \omega)^{-1} \boldsymbol{I}-(-\mathrm{i} \omega)^{-1}[-\mathrm{i}(\omega-\mathrm{Q} \mathcal{L})]^{-1} \mathrm{iQ} \mathcal{L} .
$$

The first term does not contribute because $\mathrm{Q}|\boldsymbol{A}\rangle=0$; the second term reproduces $\widehat{\boldsymbol{\Sigma}}$. Thus,

$$
\widehat{\boldsymbol{\Phi}}=\widehat{\boldsymbol{\Sigma}}-\widehat{\boldsymbol{\Sigma}} \cdot \widehat{\boldsymbol{C}},
$$

\footnotetext{
${ }^{71}$ In the following manipulations, it is easiest to use $\mathcal{L}$ everywhere, although the outermost $\mathcal{L}$ operators in the scalar products may be replaced by $\mathrm{L}$ (recall the definitions of $\mathcal{L}$ and $\mathrm{L}$ given in (3.27)).
} 
where

$$
\widehat{\boldsymbol{C}} \doteq(-\mathrm{i} \omega)^{-1}\left\langle\boldsymbol{A}|\mathcal{L} \widehat{G} \mathrm{Q} \mathcal{L}| \boldsymbol{A}^{\mathrm{T}}\right\rangle \cdot \boldsymbol{M}^{-1} .
$$

Insert the identity $\mathrm{P}+\mathrm{Q}=1$ after the first $\mathcal{L}$. The $\mathrm{P}$ term introduces the frequency matrix, and the $\mathrm{Q}$ term reproduces $\widehat{\boldsymbol{\Phi}}$ :

$$
\widehat{\boldsymbol{C}}=(-\mathrm{i} \omega)^{-1} \boldsymbol{\Omega} \cdot\left\langle\boldsymbol{A}|\widehat{G} \mathrm{Q} \mathcal{L}| \boldsymbol{A}^{\mathrm{T}}\right\rangle \cdot \boldsymbol{M}^{-1}+(-\mathrm{i} \omega)^{-1} \widehat{\boldsymbol{\Phi}} .
$$

Write

$$
\widehat{G}=(-\mathrm{i} \omega)^{-1}-(-\mathrm{i} \omega)^{-1} \widehat{G} \mathrm{i} \mathcal{L} .
$$

Again the first term does not contribute; the second term reproduces $\widehat{\boldsymbol{C}}$. Upon solving (G52) for $\boldsymbol{C}$, one finds

$$
\widehat{\boldsymbol{C}}=[-\mathrm{i}(\omega \boldsymbol{I}-\boldsymbol{\Omega})]^{-1} \cdot \widehat{\boldsymbol{\Phi}}
$$

then solving (G 47) for $\widehat{\boldsymbol{\Phi}}$ leads to

$$
\widehat{\boldsymbol{\Phi}}=\widehat{\boldsymbol{\Sigma}}-\widehat{\boldsymbol{\Sigma}} \cdot[-\mathrm{i}(\omega \boldsymbol{I}-\boldsymbol{\Omega}+\mathrm{i} \widehat{\boldsymbol{\Sigma}})]^{-1} \cdot \widehat{\boldsymbol{\Sigma}} .
$$

This generalizes (G 40). If the matrices are invertible, (G 55) can be written as

$$
\widehat{\boldsymbol{\Phi}}^{-1}=\widehat{\boldsymbol{\Sigma}}^{-1}+[-\mathrm{i}(\omega \boldsymbol{I}-\boldsymbol{\Omega})]^{-1},
$$

which generalizes (G 41).

An alternative way of writing (G55) is

$$
\widehat{\boldsymbol{\Phi}}=\widehat{\boldsymbol{\Sigma}} \cdot[-\mathrm{i}(\omega \boldsymbol{I}-\boldsymbol{\Omega}+\mathrm{i} \widehat{\boldsymbol{\Sigma}})]^{-1} \cdot[-\mathrm{i}(\omega \boldsymbol{I}-\boldsymbol{\Omega})] .
$$

Upon recalling that the determinant of a product is the product of the determinants, one finds

$$
\operatorname{det}(\widehat{\boldsymbol{\Phi}})=[\operatorname{det}(\widehat{\boldsymbol{\Sigma}})][\operatorname{det}(-\mathrm{i}(\omega \boldsymbol{I}-\boldsymbol{\Omega}+\mathrm{i} \widehat{\boldsymbol{\Sigma}}))]^{-1}[\operatorname{det}(-\mathrm{i}(\omega \boldsymbol{I}-\boldsymbol{\Omega}))] .
$$

Thus, $\operatorname{det}\left(\widehat{\boldsymbol{\Phi}}\left(\omega_{i}\right)\right)=0$ for any eigenvalue $\omega_{i}$ of $\boldsymbol{\Omega}$. This generalizes (G 42). (For the classical Langevin problem, $\Omega$ vanishes, ${ }^{72}$ so $\widehat{\boldsymbol{\Phi}}$ vanishes at $\omega=0$.)

Although the transport processes are naturally represented by the long-wavelength, low-frequency limit of $\widehat{\boldsymbol{\Sigma}}$, it is also possible to extract them from $\widehat{\boldsymbol{\Phi}}$ if one is careful. The Langevin example shows that $\widehat{\boldsymbol{\Phi}}(0)$ is unrelated to $\widehat{\boldsymbol{\Sigma}}(0)$ for the special case of vanishing frequency matrix, and the scalar version of (G 57) for non-zero $\Omega$ shows that $\widehat{\boldsymbol{\Phi}}(0)$ has at best a peculiar relation to $\widehat{\boldsymbol{\Sigma}}(0)$. To understand how to proceed, first consider the classical neutral-fluid or OCP cases, for which $\widehat{\boldsymbol{\Sigma}}(0)=k^{2} \boldsymbol{D}$ (where $\boldsymbol{D}$ is the matrix of transport coefficients) and $\boldsymbol{\Omega} \propto k$. It follows that

$$
\boldsymbol{D}=\lim _{k, \omega \rightarrow 0} k^{-2} \widehat{\boldsymbol{\Sigma}}_{k}(\omega)=\lim _{\omega \rightarrow 0} \lim _{k \rightarrow 0} k^{-2} \widehat{\boldsymbol{\Phi}}_{k}(\omega)
$$

\footnotetext{
${ }^{72} \mathrm{~A}$ well-known generalization is the harmonically bound Brownian particle, which does have a non-zero $\boldsymbol{\Omega}$.
} 
The order of limits is immaterial for $\widehat{\boldsymbol{\Sigma}}$, but the $k \rightarrow 0$ limit must be taken first for $\widehat{\boldsymbol{\Phi}}$. (In (G 57), the $k \rightarrow 0$ limit removes the $\boldsymbol{\Omega}$ and the second $\widehat{\boldsymbol{\Sigma}}$. The terms involving $\omega$ then cancel, after which the $\omega \rightarrow 0$ limit evaluates $k^{-2} \widehat{\boldsymbol{\Sigma}}(\mathbf{0}, \omega)$ at $\omega=0$.) That the ordered limit must be taken for $\widehat{\boldsymbol{\Phi}}$ agrees with the results of Kadanoff \& Martin (1963).

This discussion clarifies an argument used by Brey et al. (1981). They work in the space-time domain rather than with Fourier transforms. They derive an expression equivalent to $(\mathrm{G} 47)$, then assert $^{73}$ that the term $\langle\boldsymbol{A}| \mathrm{LQG}_{\mathrm{Q}} \mathrm{iP}$ is negligible through second order in the gradients because (when $\mathrm{L}$ is proportional to a gradient) it involves $G_{Q}$ evaluated to zeroth order in the gradients (namely the identity operator) and $\mathrm{QP}=0$. Thus, they are first taking the limit of small gradients (i.e. $k \rightarrow 0$ ). Then they assert that 'the time integral can be extended to infinity' (i.e. they take the $\omega \rightarrow 0$ limit after passing to the limit of infinite system size).

The arguments of Brey et al. fail in the multispecies case where L contains the collision operator, first because $\widehat{\mathrm{C}}$ does not tend to zero with the gradients, second because $\mathrm{QCQ} \neq \widehat{\mathrm{C}}$. Thus, the unmagnetised Braginskii transport coefficients involve $(\mathrm{Q} \widehat{\mathrm{C}} \mathrm{Q})^{-1}$ rather than $\widehat{\mathrm{C}}^{-1}=\int_{0}^{\infty} \mathrm{d} \bar{\tau} \mathrm{e}^{-\widehat{\mathrm{C}} \bar{\tau}}$.

In the multispecies case, the frequency matrix contains terms that are of the order of the collision frequency and do not vanish with $k$. That would not be a problem if $\widehat{\Sigma}_{k}$ would vanish with $k$, in which case the ordered limit (G 59) would still work. However, we know from the discussion at the end of $\S 3.2$ that the physics of the high-energy tail is contained in $\widehat{\Sigma}_{k}$, and the contribution of that tail to the effective collision frequency does not vanish with $k$. Thus, one has a situation analogous to the Langevin model discussed at the beginning of the section. One could then resort to the generalization of $(\mathrm{G} 44)$, which from $(\mathrm{G} 56)$ is

$$
\boldsymbol{D}^{-1}=\lim _{\omega, k \rightarrow 0} k^{2}\left\{\widehat{\boldsymbol{\Phi}}^{-1}-[-\mathrm{i}(\omega \boldsymbol{I}-\boldsymbol{\Omega})]^{-1}\right\}
$$

provided that the matrices are invertible.

\section{G.4. Projection-operator analysis of the Brownian test particle}

Analysis of the long-time statistical dynamics of the classical Brownian test particle by projection-operator techniques provides probably the simplest non-trivial example of the methodology and also provides further insights into the interactions between null spaces and multidimensional projections. To the stochastic momentum equation (G 32), adjoin

$$
\frac{\mathrm{d} \widetilde{x}}{\mathrm{~d} t}=\widetilde{v}=\widetilde{p} / M .
$$

It is then well known that for times much longer than the collision time $v^{-1}$ $x$-space diffusion ensues:

$$
\left\langle\delta x^{2}\right\rangle \rightarrow 2 D t, \quad \text { where } D=v_{T}^{2} / \nu .
$$

The exact solution of the (linear) Langevin system can also be obtained for all times from one of two equivalent methods: (i) Recognize that $\widetilde{x}$ and $\widetilde{v}$ are jointly Gaussian because they solve linear ODEs driven by Gaussian noise, then calculate

\footnotetext{
${ }^{73}$ Actually, they expand $\widehat{\boldsymbol{\Sigma}}$ in terms of $\widehat{\boldsymbol{\Phi}}$, so the roles of $\mathrm{G}_{\mathrm{Q}}$ and $\mathrm{G}$ are reversed.
} 
the conditional means and variances by directly solving the ODEs and appropriately averaging the solutions; (ii) solve the equivalent Fokker-Planck equation

$$
\frac{\partial f(x, v, t)}{\partial t}+v \frac{\partial f}{\partial x}=-\widehat{\mathrm{C}} f
$$

where $\widehat{\mathrm{C}}$ is given by (B 35). ${ }^{74}$

\section{G.4.1. Projection into the density subspace}

First consider how the $x$-space diffusion result (G62) emerges from the natural hydrodynamic projection-operator formalism. It is not difficult to show that $\widehat{\mathrm{C}}$ possesses a single null eigenvalue, with eigenvector $\left|\mathrm{He}_{0}\right\rangle=|1\rangle$ associated with probability (or density) conservation. This motivates the projection of (G63) onto the 1-D density subspace, namely $\mathrm{P}=|1\rangle\langle 1|$. The frequency 'matrix' $\Omega=k\langle 1|v| 1\rangle$ vanishes by symmetry (or equivalently by the orthogonality of $\mathrm{He}_{0}$ and $\mathrm{He}_{1}$ ). Because the eigenvalues of $\widehat{\mathrm{C}}$ are the Hermite polynomials and the $\mathrm{Q}$ projection excludes the null space, the Markovian approximation is valid for time longer than $v^{-1}$, and one may use the specialization of the result (2.54) to a single density diffusion coefficient. One obtains

$$
\partial_{t} n+k^{2} D n=0
$$

where

$$
D=\left\langle v \mathrm{Q}\left|(\mathrm{Q} \widehat{\mathrm{C}})^{-1}\right| \mathrm{Q} v\right\rangle=\left\langle v\left|\widehat{\mathrm{C}}^{-1}\right| v\right\rangle
$$

and I used the facts that $\mathrm{PC}=\widehat{\mathrm{CP}}=0$ and $\mathrm{Q}|v\rangle=(1-\mathrm{P})|v\rangle=|v\rangle .{ }^{75}$ The solution of the differential equation

$$
\widehat{\mathrm{C}}|\psi\rangle=|v\rangle
$$

is easy to obtain and is just $|\psi\rangle=|v\rangle / \nu$; then

$$
D=\langle v \mid v\rangle / v=v_{T}^{2} / v,
$$

in agreement with the known result (G62).

\section{G.4.2. Projection into a multidimensional subspace}

Suppose that one does not recognize the one-dimensional nature of the null space. What would happen if one would use a higher-dimensional projection? ${ }^{76}$ As an example, I shall consider a 2-D projection into the density and velocity subspaces. Namely, with velocities normalized to $v_{T}$, project with $\mathrm{P}=|1\rangle\langle 1|+| v\rangle\langle v|$. The frequency matrix becomes

$$
\boldsymbol{\Omega}=k\left(\begin{array}{ll}
\langle 1|v| 1\rangle & \langle 1|v| v\rangle \\
\langle v|v| 1\rangle & \langle v|v| v\rangle
\end{array}\right)=k\left(\begin{array}{ll}
0 & 1 \\
1 & 0
\end{array}\right) .
$$

\footnotetext{
${ }^{74}$ Equation (G63) is a special case of a class of PDEs that can be solved exactly to yield a multivariate Gaussian, as discussed by van Kampen (1981, § VIII.6). The key features are (i) the first-order terms are linear in the independent variables, and (ii) the second-order terms involve only constant coefficients.

${ }^{75}$ Note that the last form of $(\mathrm{G} 65)$ does not follow from the previous one by setting $\mathrm{Q}^{-1} \mathrm{Q}=1$, since projection operators other than the identity are not invertible (as follows from the property $\mathrm{Q}^{2}=\mathrm{Q}$ ).

${ }^{76}$ Higher-order moment closures were discussed by Grad (1958); a popular one is his system of 13 moment equations. Grad's method is discussed in the useful textbook on plasma kinetic theory by Ecker (1972, §IV.2).
} 
The Markovian approximation is even better justified (the $\mathrm{Q}$ projection comprises Hermite polynomials of order 2 and above). The only surviving transport coefficient can easily be shown to be $\eta_{v}^{v} / k^{2} \equiv \mu$; it is easy to calculate, but its value will not be needed to lowest order in the hydrodynamic limit. The projected system of equations takes the form

$$
\begin{gathered}
\partial_{t} n+\mathrm{i} k u=0, \\
\partial_{t} u+\mathrm{i} k n+k^{2} \mu u=-v u,
\end{gathered}
$$

where the right-hand side of the last equation arises because the collision operator does not conserve momentum. The dispersion relation is

$$
\lambda^{2}+\left(v+k^{2} \mu\right) \lambda+k^{2}=0 .
$$

For $k^{2} \rightarrow 0$, the $1-0$ balance is, upon undoing the velocity normalization, $\lambda=-k^{2} D$ with $D$ as in (G67); this is the density diffusion mode. The $2-1$ balance is $\lambda=-v$, which describes momentum relaxation.

Thus, although in this example it is natural and optimally efficient to project into the 1-D density subspace, no harm is done by employing a higher-dimensional projection $^{77}$ as long as part of it projects into the complete null space of the collision operator. If such a projection is treated in a mathematically consistent way, the long-time transport must still emerge, though it is likely to arise in ways that are mathematically different depending on the projection. For example, in the 1-D projection diffusion is represented by the dissipative $\eta$ coefficient, while in the density plus momentum projection the $\eta$ coefficient vanishes in the density equation and is subdominant in the momentum equation; density diffusion arises from the coupling between density and the momentum-dissipation effect represented by the explicit momentum projection of the collision operator, which does not conserve momentum.

If one combines the insights of the present example with the caveats from the earlier examples in this appendix, which show that it is problematical to project into a subspace of dimension lower than that of the null space, one deduces that for a Markovian treatment of long-time transport based on a collisional kinetic equation, one must use a projection that at least spans all of the null space of the collision operator but may be of higher dimensionality if additional information is desired. This is a satisfying consistency. However, although the projection method is flexible and intuitive, a solid physical understanding of the effects to be described is essential to a successful exploitation of the techniques.

\section{REFERENCES}

Alder, B. J. \& WAinwright, T. E. 1970 Decay of the velocity autocorrelation function. Phys. Rev. A 1, 18-21.

BALescu, R. 1975 Equilibrium and Nonequilibrium Statistical Mechanics. Wiley; reprinted by Krieger Publishing Co., Malabar, Florida, 1991.

Belova, E. V. 2001 Nonlinear gyroviscous force in a collisionless plasma. Phys. Plasmas 8, 3936-3944.

Berne, B. J. 1971 Time-dependent properties of condensed media. In Physical Chemistry: An Advanced Treatise (ed. H. Eyring, D. Henderson \& W. Jost), vol. VIIIB, pp. 539-716. Academic.

${ }^{77}$ Note that the exact solution of the Fokker-Planck equation corresponds to an infinite-dimensional projection. 
BIXON, M. \& ZWANZIG, R. 1969 Boltzmann-Langevin equation and hydrodynamic fluctuations. Phys. Rev. 187, 267-272.

BRAgINSKII, S. I. 1957 Transport phenomena in a completely ionized two-temperature plasma. Zh. Eksp. Teor. Fiz. 33, 459-472 [Sov. Phys. JETP 6, 358-369 (1958)].

BRAginskit, S. I. 1965 Transport processes in a plasma. In Reviews of Plasma Physics (ed. M. N. Leontovich), vol. 1, pp. 205-311. Consultants Bureau.

Brey, J. J., ZWANZig, R. \& Dorfman, J. R. 1981 Nonlinear transport equations in statistical mechanics. Physica A 109, 425-444.

CAsimir, H. B. G. 1945 On Onsager's principle of microscopic reversibility. Rev. Mod. Phys. 17, 343.

Catto, P. J. \& Simakov, A. N. 2004 A drift ordered short mean free path description for magnetized plasma allowing strong spatial anisotropy. Phys. Plasmas 11, 90-102.

ChAPMAN, S. 1916 On the law of distribution of velocities, and on the theory of viscosity and thermal conduction, in a non-uniform simple monatomic gas. Phil. Trans. R. Soc. Lond. A 216, 279-348.

Chapman, S. \& Cowling, T. G. 1952 The Mathematical Theory of Non-Uniform Gases, 2nd edn. Cambridge University Press.

Chow, C. C. 2007 Multiple scale analysis. Scholarpedia 2, 1617.

DAWson, J. \& OBERMAN, C. 1962 High-frequency conductivity and the emission and absorption coefficients of a fully ionized plasma. Phys. Fluids 5, 517-524.

Dawson, J. M. 1968 Radiation from plasmas. In Advances in Plasma Physics (ed. A. Simon \& W. B. Thompson), vol. 1, pp. 1-66. Wiley.

Diamond, P. H., Itoh, S.-I. \& ITOH, K. 2010 Modern Plasma Physics, Physical Kinetics of Turbulent Plasmas, vol. 1. Cambridge University Press.

ECKer, G. 1972 Theory of Fully Ionized Plasmas. Academic Press.

Enskog, D. 1917 The kinetic theory of phenomena in fairly rare gases. PhD thesis, Upsala.

Fecko, M. 2006 Differential Geometry and Lie Groups for Physicists. Cambridge University Press.

FEYNMAN, R. P. 1951 An operator calculus having applications in quantum electrodynamics. Phys. Rev. 84, 108-128.

Forster, D. \& Martin, P. C. 1970 Kinetic theory of a weakly coupled fluid. Phys. Rev. A 2, $1575-1590$.

FoX, R. F. 1978 Gaussian stochastic processes in physics. Phys. Rev. 48, 179-283.

GORBAN, A. N. \& KARLIN, I. 2014 Hilbert's 6th problem: exact and approximate hydroynamic manifold for kinetic equations. Bull. Am. Math. Soc. 51 (2), 186-246.

GotTFrIED, K. 1966 Quantum Mechanics. Volume I: Fundamentals. Benjamin.

GRAD, H. 1958 Principles of the kinetic theory of gases. In Handbuch der Physik, vol. XII, pp. 205-294. Springer.

GREEN, M. S. 1954 Markoff random processes and the statistical mechanics of time-dependent phenomena. II. Irreversible processes in fluids. J. Chem. Phys. 22, 398-413.

Hammett, G. W. \& Perkins, F. W. 1990 Fluid moment models for Landau damping with application to the ion-temperature-gradient instability. Phys. Rev. Lett. 64, 3019-3022.

HaZeltine, R. D. 2006 Coulomb collision operator. Tech. Rep., University of Texas, http://w3fusion.ph.utexas.edu/ifs/ifsreports/1140_Hazeltine.pdf.

Helander, P. \& Sigmar, D. J. 2002 Collisional Transport in Magnetized Plasmas. Cambridge University Press.

Hinton, F. L. 1970 Nonequilibrium theory of fluid fluctuations. Phys. Fluids 13, 857-866.

KadAnoff, L. P. \& Martin, P. C. 1963 Hydrodynamic equations and correlation functions. Ann. Phys. 24, 419-469.

Kaufman, A. N. 1960 Plasma viscosity in a magnetic field. Phys. Fluids 3, 610-616.

Kent, A. \& TAYlor, J. B. 1969 Plasma fluctuations and convective modes. Phys. Fluids 12, 209-212.

KrommeS, J. A. 1975 On renormalized kinetic theories of anomalous transport due to hydrodynamic fluctuations in strongly magnetized plasma. $\mathrm{PhD}$ thesis, Princeton University. 
Krommes, J. A. 2002 Fundamental statistical theories of plasma turbulence in magnetic fields. Phys. Rep. 360, 1-352.

Krommes, J. A. 2012 The gyrokinetic description of microturbulence in magnetized plasmas. Annu. Rev. Fluid Mech. 44, 175-201.

Krommes, J. A. 2015 A tutorial introduction to the statistical theory of turbulent plasmas, a half century after Kadomtsev's Plasma Turbulence and the resonance-broadening theory of Dupree and Weinstock. J. Plasma Phys. 81, 1-80.

Krommes, J. A. 2018 a An introduction to the physics of the Coulomb logarithm with emphasis on quantum-mechanical effects, arXiv:1806.04990 (8 pages).

Krommes, J. A. $2018 b$ Projection-operator methods for classical transport in magnetized plasmas. II. Nonlinear response and the Burnett equations, arXiv:1711.02202 (100 pages).

Krommes, J. A. \& HU, G. 1993 General theory of Onsager symmetries for perturbations of equilibrium and nonequilibrium steady states. Phys. Fluids B 5, 3908-3941.

Krommes, J. A., LeE, W. W. \& Oberman, C. 1986 Equilibrium fluctuation energy of gyrokinetic plasma. Phys. Fluids 29, 2421-2425.

Krommes, J. A. \& Oberman, C. 1976 Anomalous transport due to long-lived fluctuations in plasma. Part II. Hydrodynamic contributions to transport in two-dimensional, strongly magnetized systems. J. Plasma Phys. 16, 229-260.

Kruskal, M. 1965 Asymptotology. In Plasma Physics, Seminar in Plasma Physics (Trieste, Italy, 1964), pp. 373-387. International Atomic Energy Agency.

Kubo, R. 1957 Statistical-mechanical theory of irreversible processses. I. General theory and simple applications to magnetic and conduction problems. J. Phys. Soc. Japan 12, 570-586.

Kubo, R. 1974 Response, relaxation, and fluctuations. In Lecture Notes in Physics: Transport Phenomena (ed. G. Kirczenow \& J. Marro), vol. 31, pp. 24-124. Springer.

Landau, L. D. \& Lifshitz, E. M. 1957 Hydrodynamic fluctuations. Sov. Phys. JETP 5, 512-513.

Landau, L. D. \& Lifshitz, E. M. 1987 Fluid Mechanics, 2nd edn. Pergamon; reprinted with corrections 1997.

Langevin, P. 1908 Sur la théorie du mouvement brownien. C. R. Acad. Sci. 146, 530-533; translation in D. S. Lemons and A. Gythiel, Paul Langevins 1908 paper 'On the theory of Brownian motion' ['Sur la théori du mouvement brownien,' C. R. Acad. Sci. (Paris) 146, 530-533 (1908)], Am. J. Phys. 65, 1079-1081 (1997).

LEWIS, J. D. 1967 Expansion theorem for the linearized Fokker-Planck equation. J. Math. Phys. 8, 791-798.

Martin, P. C. 1967 Sum rules, Kramers-Kronig relations, and transport coefficients in charged systems. Phys. Rev. 161, 143-155.

Martin, P. C. 1968 Measurements and correlation functions. In Many Body Physics (ed. C. deWitt \& R. Balian), pp. 37-136. Gordon and Breach.

MazenKo, G. 1973 Fully renormalized kinetic theory. I. Self-diffusion. Phys. Rev. A 7, 209-221.

MikHAǏLOVSKIĬ, A. B. 1967 Macroscropic description of a collision[al] plasma in a strong magnetic field for stability problems. Zh. Eksp. Teor. Fiz. 52, 943-954 [Sov. Phys. JETP 25, 623-630 (1967)].

Mikhalllovskiľ, A. B.\& TsyPin, V. S. 1971 Transport equations and gradient instabilities in a high pressure collisional plasma. Plasma Phys. 13, 785-798.

MikhalĺovskiǏ, A. B. \& TSYPIN, V. S. 1984 Transport equations of plasma in a curvilinear magnetic field. Beitr. Plasma Phys. 24, 335-354.

Misguich, J. H. 1975 Stochastic turbulence in Vlasov plasmas. Physica $B+C$ 79, 373-401.

Misguich, J. H. \& BALESCU, R. 1975 Re-normalized quasi-linear approximation of plasma turbulence. Part 1. Modification of the Weinstock weak-coupling limit. J. Plasma Phys. 13, 385-417.

Misner, C. W., Thorne, K. S. \& Wheeler, J. A. 1973 Gravitation. W. H. Freeman and Company. Montgomery, D. C. \& Tidman, D. A. 1964 Plasma Kinetic Theory. McGraw-Hill.

Mori, H. 1965 Transport, collective motion, and Brownian motion. Prog. Theor. Phys. 33, 423-455.

OnsAger, L. $1931 a$ Reciprocal relations in irreversible processes. I. Phys. Rev. 37, 405-426.

Onsager, L. 1931 b Reciprocal relations in irreversible processes. II. Phys. Rev. 38, 2265-2279. 
Reichl, L. E. 1998 A Modern Course in Statistical Physics, 2nd edn. Wiley. (The section on Beyond the Boltzmann Equation was radically shortened in the third and fourth editions.)

Robinson, B. B. \& Bernstein, I. B. 1962 A variational description of transport phenomena in a plasma. Ann. Phys. 18, 110-169.

Rosenbluth, M. N. \& Kaufman, A. N. 1958 Plasma diffusion in a strong magnetic field. Phys. Rev. 109, 1-5.

Rosenbluth, M. N. \& LiU, C. S. 1976 Cross-field energy transport by plasma waves. Phys. Fluids 19, $815-818$.

van Kampen, N. G. 1981 Stochastic Processes in Physics and Chemistry. North-Holland.

WAnG, M. C. \& Uhlenbeck, G. E. 1945 On the theory of the Brownian motion II. Rev. Mod. Phys. 17, 323-342; reprinted in Selected Papers on Noise and Stochastic Processes, edited by N. Wax (Dover, New York, 1954), pp. 113-132.

Weinhold, F. 1975 Metric geometry of equilibrium thermodynamics. J. Chem. Phys. 63, 2479-2483; 2484-2487; 2488-2495; 2496-2501.

Weinhold, F. 1976 Metric geometry of equilibrium thermodynamics. J. Chem. Phys. 65, 559-564.

Weinstock, J. 1969 Formulation of a statistical theory of strong plasma turbulence. Phys. Fluids 12, $1045-1058$.

Weinstock, J. 1970 Turbulent plasmas in a magnetic field - a statistical theory. Phys. Fluids 13, $2308-2316$.

ZWANZIG, R. 1961a Memory effects in irreversible thermodynamics. Phys. Rev. 124, 983-992.

ZWANZIG, R. $1961 b$ Statistical mechanics of irreversibility. In Lectures in Theoretical Physics (ed. W. E. Brittin, B. W. Downs \& J. Downs), vol. 3, pp. 106-141. Interscience.

ZwanZIG, R. 2001 Nonequilibrium Statistical Mechanics. Oxford University Press. 